\title{
WestVirginiaUniversity
}

THE RESEARCH REPOSITORY @ WVU

Graduate Theses, Dissertations, and Problem Reports

2009

\section{Agent based modeling of power distribution systems}

Sridhar Chouhan

West Virginia University

Follow this and additional works at: https://researchrepository.wvu.edu/etd

\section{Recommended Citation}

Chouhan, Sridhar, "Agent based modeling of power distribution systems" (2009). Graduate Theses, Dissertations, and Problem Reports. 1996.

https://researchrepository.wvu.edu/etd/1996

This Thesis is protected by copyright and/or related rights. It has been brought to you by the The Research Repository @ WVU with permission from the rights-holder(s). You are free to use this Thesis in any way that is permitted by the copyright and related rights legislation that applies to your use. For other uses you must obtain permission from the rights-holder(s) directly, unless additional rights are indicated by a Creative Commons license in the record and/ or on the work itself. This Thesis has been accepted for inclusion in WVU Graduate Theses, Dissertations, and Problem Reports collection by an authorized administrator of The Research Repository @ WVU. For more information, please contact researchrepository@mail.wvu.edu. 


\title{
Agent Based Modeling of Power Distribution Systems
}

\author{
by \\ Sridhar Chouhan \\ Thesis submitted to the \\ College of Engineering and Mineral Resources \\ at West Virginia University \\ in partial fulfillment of the requirements \\ for the degree of \\ Master of Science \\ in \\ Electrical Engineering
}

Prof. Ali Feliachi, Ph.D., Chair

Prof. Daryl S Reynolds, Ph.D.

Prof. Muhammad A. Choudhry, Ph.D.

Lane Department of Computer Science and Electrical Engineering

Morgantown, West Virginia

2009

Keywords: Multi-Agent System, Fault Detection, Reconfiguration, DEW@, JADE, Power World Simulator.

Copyright 2009 Sridhar Chouhan 


\title{
ABSTRACT
}

\section{Agent Based Modeling of Power Distribution Systems}

\author{
by \\ Sridhar Chouhan \\ Master of Science in Electrical Engineering \\ West Virginia University \\ Professor Ali Feliachi, Ph.D., Chair
}

The electric power system is a very vast network and becoming more complex each day. The traditional vertically monopolistic structure has been deregulated and replaced by gencos, transcos and, discos; increasing the power system intricacy. During the past few decades there has been remarkable development in software and hardware technologies for the analysis and design activities in power system planning, operation, and control. However, much still depends on the judgment of human experts. A single fault in power system can lead to multiple faults and can collapse the whole system. Power System needs a more decentralized control mechanism for solving these problems. One novel solution would be Multi-agent Systems. A Multi-agent system is a collection of agents, which perceives the system changes and acts on the system in order to achieve its goals. Recent technology developments in the area of Multi-agent systems making it a viable solution for today's complicated power network.

A Multi-agent system model is developed for fault detection and reconfiguration in this thesis work. These models are developed based on graph theory tree models and mathematical models. A set of objective functions are specified in the mathematical model for the restoration of the network.

The agent platform for the fault detection is developed by Java Agent Development Framework. The restoration algorithm is programmed in MATLAB and applied to the distribution system modeled in the commercial software, Distributed Engineering Workstation and Power World Simulator. The test system in this thesis is, a distribution system developed by Southern California Edison called Circuit of the Future.

The Multi-agent system can detect the fault precisely and reconfigures the circuit using the reconfiguration algorithm. The reconfiguration will happen in a way that it always try to supply all the critical loads in the network. When there are multiple solutions available for reconfiguration, the one with good voltage profile and less power loss is selected as the solution. The algorithm makes use of shunt compensation and priority based load shedding in order to control the voltage across the network. Agents make use of learning to speed up the reconfiguration process. 


\section{DEDICATION}

I would like to dedicate this thesis to my parents,

who carved me to whatever little I am today,

$\&$

My adorable cousin, Koneri Srinivas,

who helped me in each mode of my life.

$\star \star \star$

Grand pa, you are deeply missed! 
The only living gods on earth are, 'Parents' 


\section{ACKNOWLEDGEMENTS}

After two years of my degree, I have learned one thing - I could never have completed my research work and the thesis dissertation, without the support and encouragement of many people around me. First of all, I would like to thank my esteemed mentor and advisor, Dr. Ali Feliachi. I owe him so much. He has given me a chance to continue my work in the field of power systems, which is actually my passion. He taught me much of what I know today and guided me throughout my research work. I would also like to thank rest of my thesis committee, Dr. Muhammad A Choudhry and Dr. Daryl S Reynolds, for their support and advice. I would like to thank Dr. Hong-Jian Lai for helping me out with the mathematical modeling used in this thesis work.

I extend the appreciation to Professor Hui Wan, all other faculty members in APERC and the staff in LDCSEE for their encouragement and support. I would also like to express my thanks to my friends in APERC for the healthy discussions regarding my research work. A special thanks to Ms. Koushaly Nareshkumar for helping me in understanding Multi-agent concepts related to power distribution systems. For assistance in JADE programming, my thanks are extended to Ms. Summiya Moheuddin. I've been fortunate enough to have a great group of friends at WVU. I would like to thank all of them for making my master's life joyful and memorable. Specially, I would like to thank my best friends Usha, Navya, and all my roommates, who were always there for me when I needed moral support.

I would like to thank my family for their unending support and love from childhood to now. Without their support I couldn't have made it through this process or any of the tough times in my life. My sincere gratitude goes to my cousin for helping and motivating me throughout my life. My humble pranam goes to the almighty god for being with me as a guiding light throughout my life.

This work was supported in part by grants from the US DEPSCoR/ONR grant No. N00014-03-1-0660 and the US DoE grant No. DE-FC26-06NT42793. 


\section{CONTENTS}

ABSTRACT …

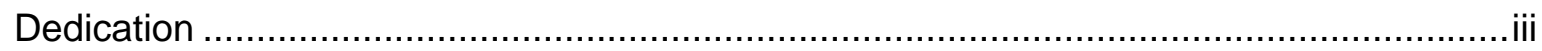

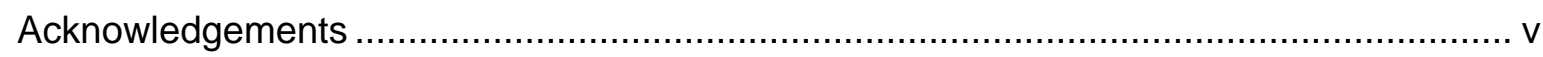

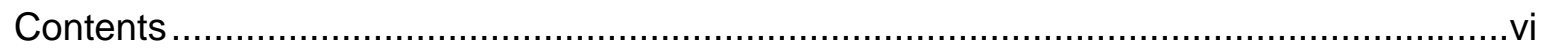

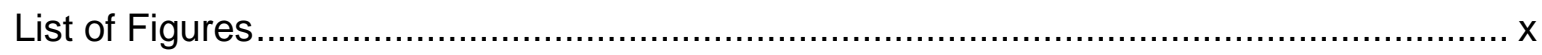

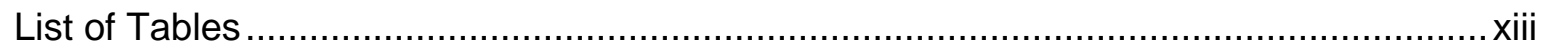

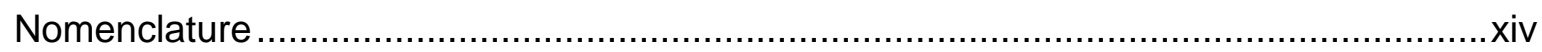

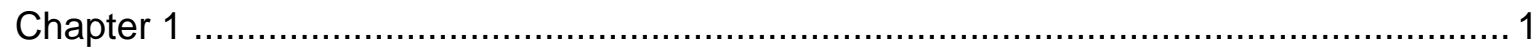

INTRODUCTION

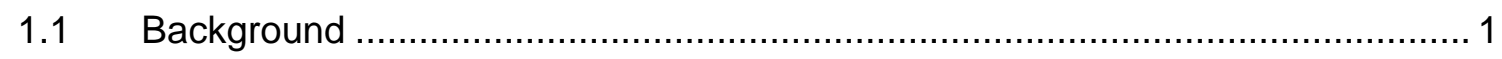

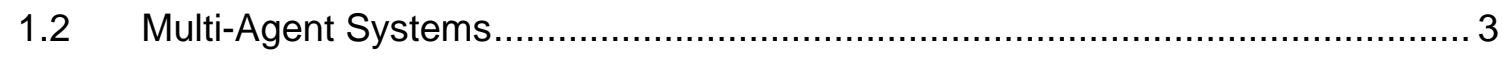

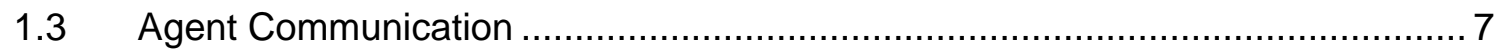

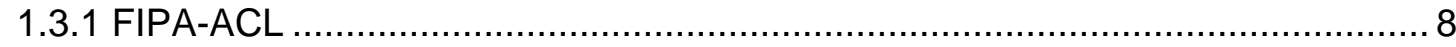

1.3.2 Knowledge Query and Manipulation Language (KQML) ............................... 10

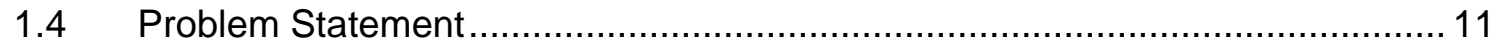

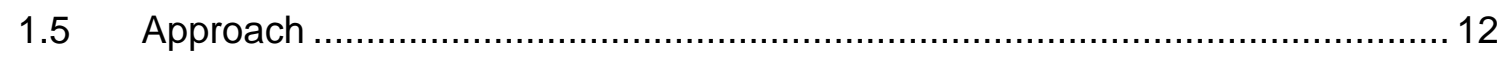

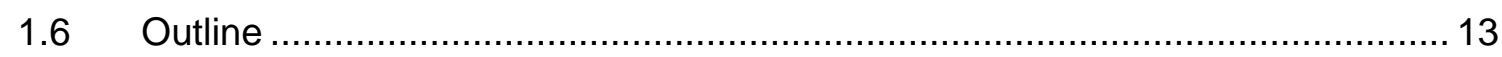

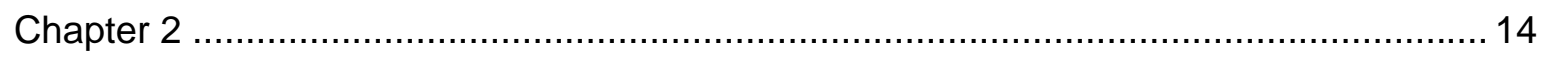




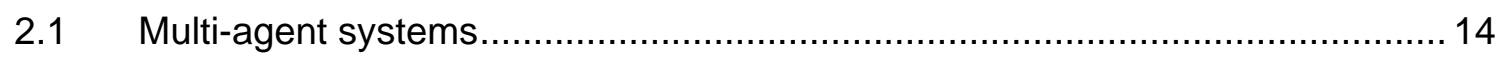

2.2 MAS in Automatic Fault Detection and Reconfiguration............................... 16

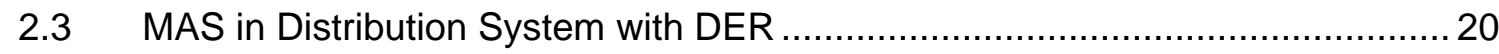

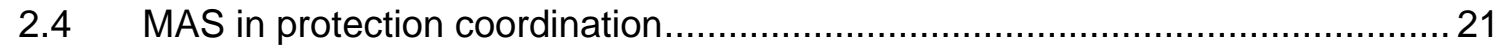

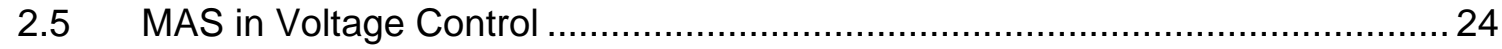

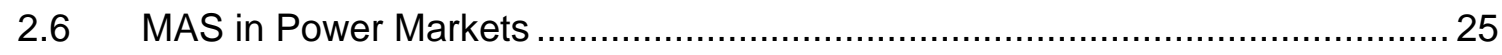

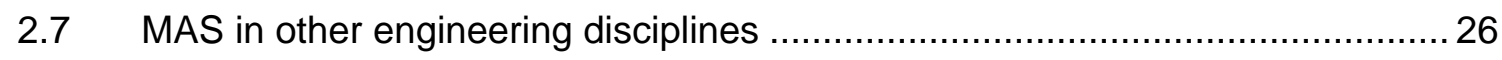

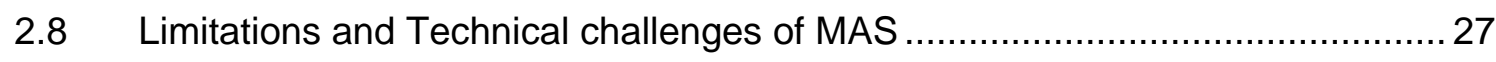

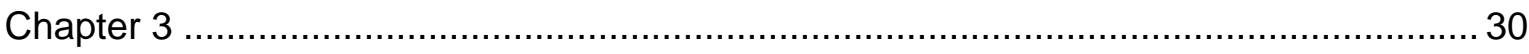

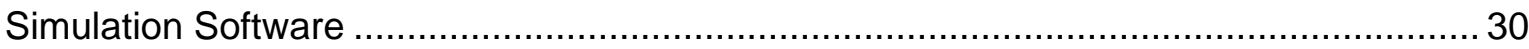

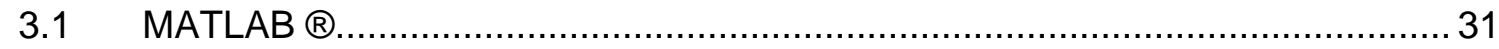

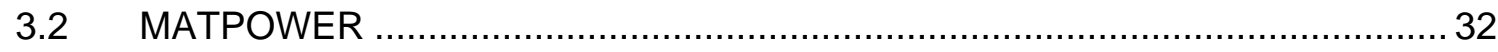

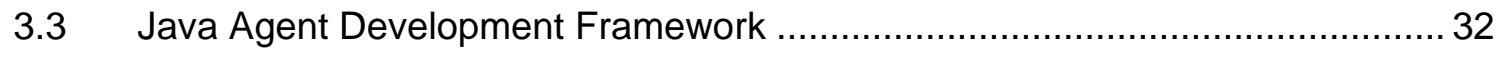

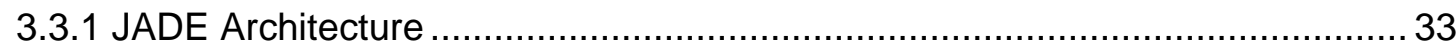

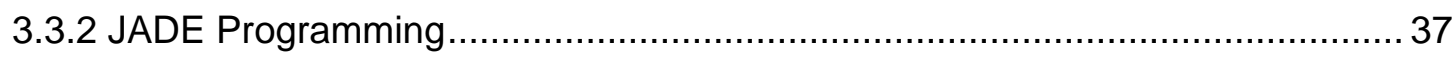

3.4 Distributed Engineering Workstation@ (DEW) ................................... 37

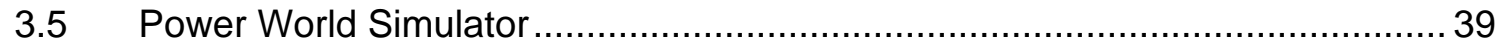

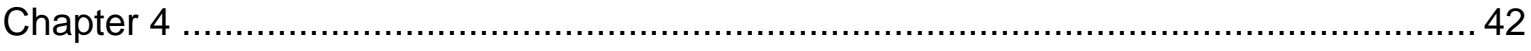

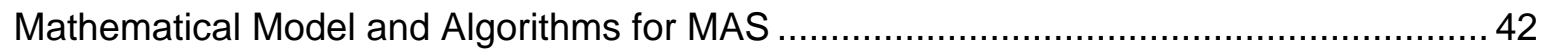

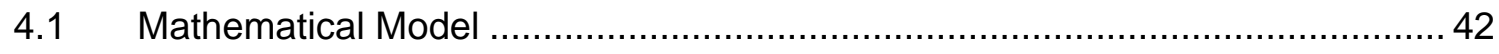




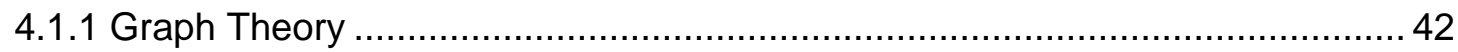

4.1.2 Graph Theory representation of Power System ................................... 43

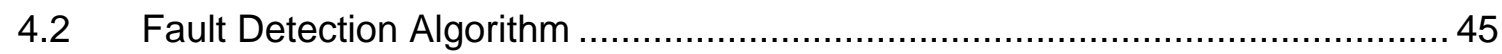

$4.3 \quad$ Fault Reconfiguration \& Restoration Algorithm ............................................ 45

4.3.1 Reconfiguration algorithm for single fault ............................................ 46

4.3.2 Reconfiguration algorithm for two faults ................................................... 46

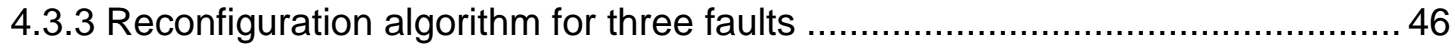

4.3.4 Objective functions and Constraints ................................................... 48

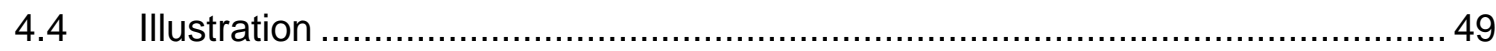

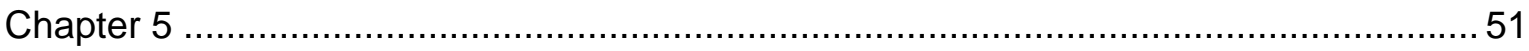

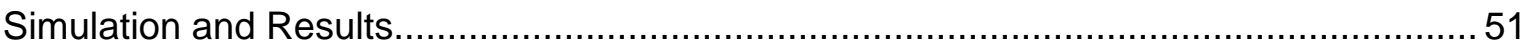

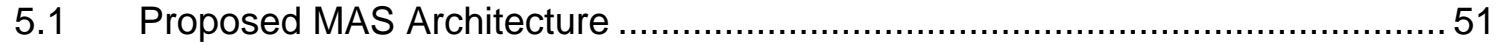

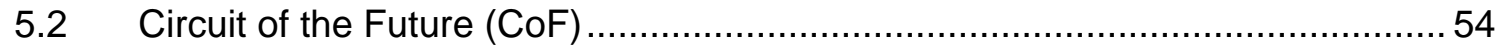

$5.3 \quad$ Fault Detection and Reconfiguration Algorithm ....................................... 57

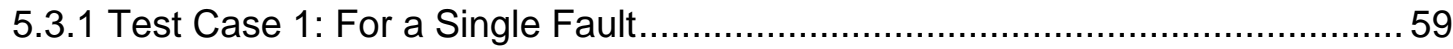

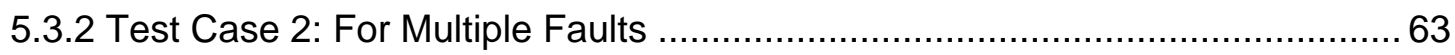

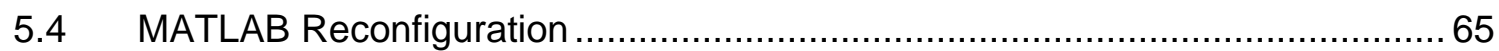

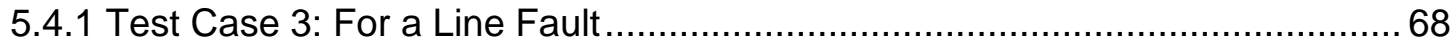

5.4.2 Test Case 4: For two Line Faults ............................................................. 73

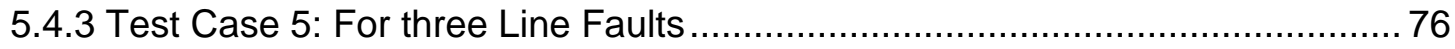

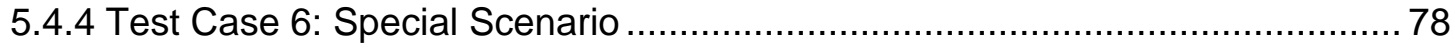




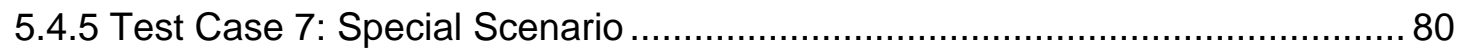

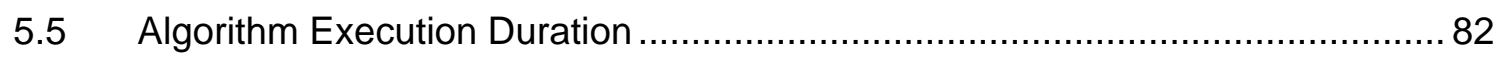

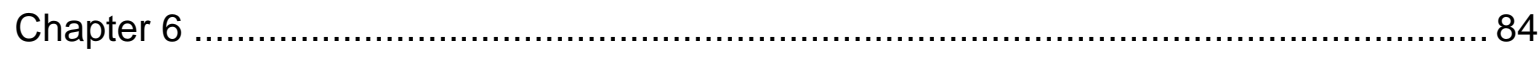

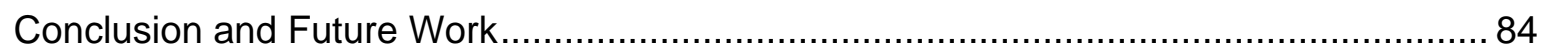

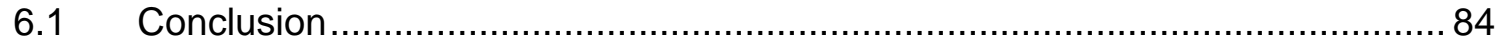

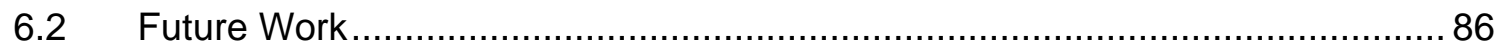

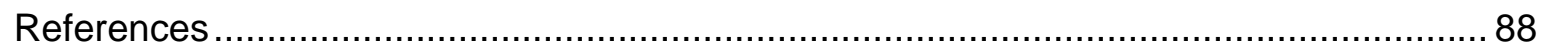




\section{LIST OF FIGURES}

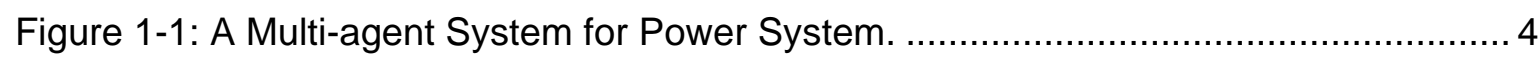

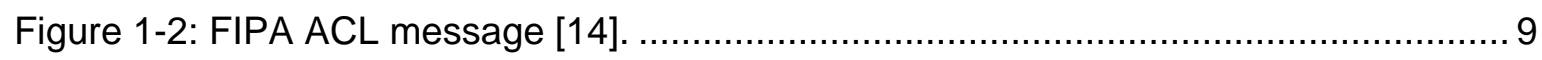

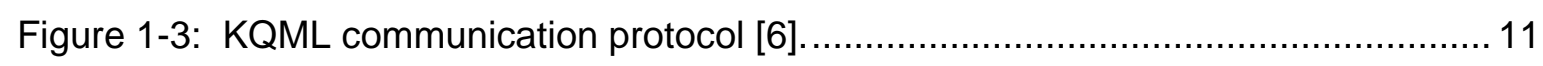

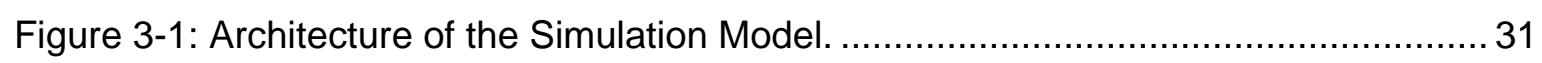

Figure 3-2: Relationship between main architectural elements [67] ........................... 34

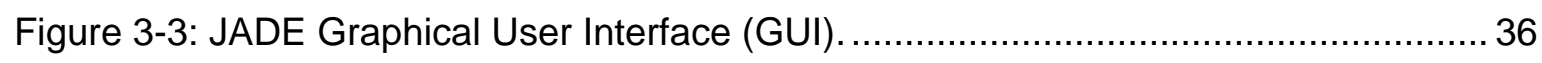

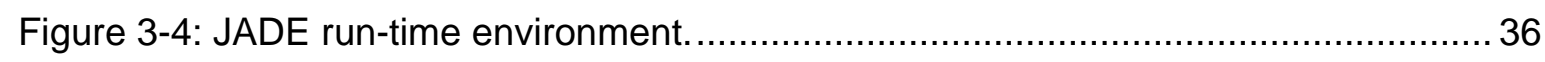

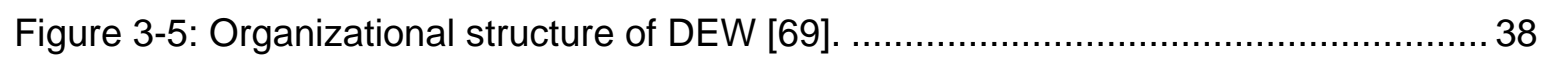

Figure 3-6: Distributed Engineering Workstation's Working Environment..................... 39

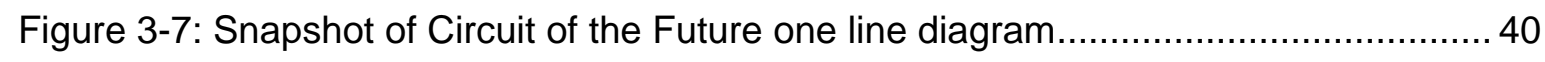

Figure 3-8: Snapshot of Model Explorer of Power World Simulator [70]..................... 41

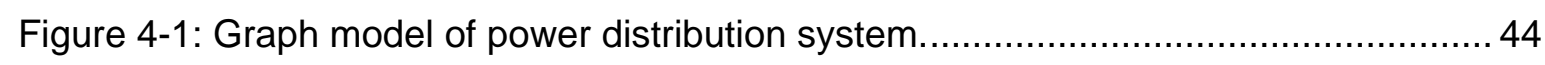

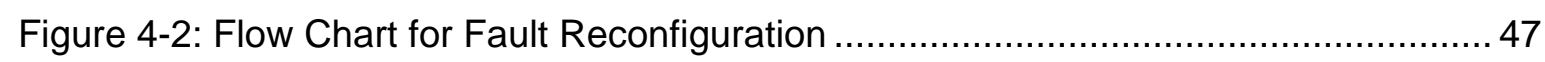

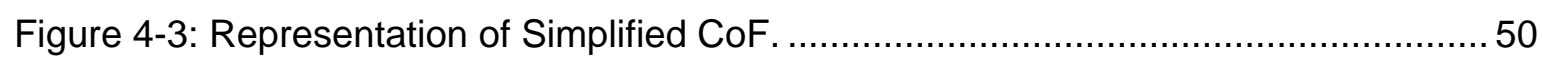

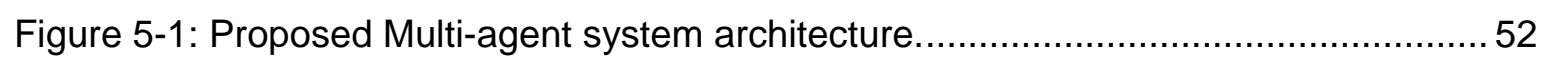

Figure 5-2: Global Agent (GAG) functionalities. .............................................. 53

Figure 5-3: The Circuit of the Future modeled in DEW [2] ..................................... 55

Figure 5-4: The Simplified CoF modeled in Power World Simulator. ............................. 56

Figure 5-5: Flow chart for Fault Detection and Reconfiguration.............................. 58

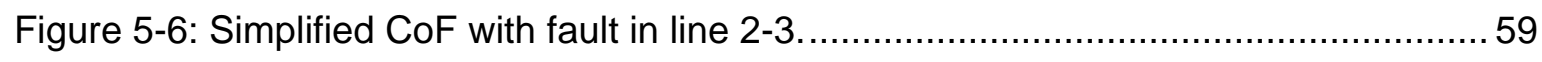

Figure 5-7: Power World Simulator's Load flow report for line fault at line 2-3.............. 60

Figure 5-8: JADE message passing between agents for line fault at line $2-3 \ldots \ldots \ldots \ldots \ldots . . . . .60$ 


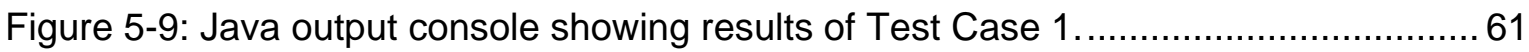

Figure 5-10: MATLAB plot depicting reconfiguration network for fault at line 2-3.......... 62

Figure 5-11: JADE message passing between agents for line faults at line 1-2 and 1-5. . 63

Figure 5-12: Java output console showing results of Test Case 2. 63

Figure 5-13: MATLAB plot depicting reconfiguration network for fault at line 1-2 and 1-5.64

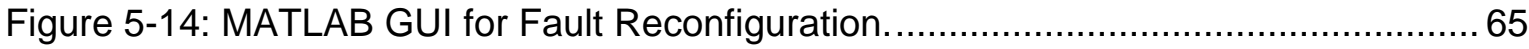

Figure 5-15: MATLAB plot depicting basic Simplified CoF.................................... 67

Figure 5-16: MATLAB plot depicting voltage profile with $S_{1}$ closed for line fault at 1-2 $\ldots . .68$

Figure 5-17: MATLAB plot depicting voltage profile with $S_{2}$ closed for line fault at 1-2.... 69

Figure 5-18: MATLAB plot depicting voltage profile with $S_{3}$ closed for line fault at 1-2.... 69

Figure 5-19: MATLAB plot depicting voltage profile with $S_{1} \& S_{5}$ closed for line fault at 1-2.

70

Figure 5-20: MATLAB plot depicting voltage profile with $S_{2} \& S_{5}$ closed for line fault at 1-2.

Figure 5-21: MATLAB plot depicting reconfiguration network for fault at line 1-2.

Figure 5-22: Plot depicting voltage profile with $S_{1}, S_{5} \& S_{7}$ closed for line faults at 1-2 \&

14-15, without voltage control.

Figure 5-23: Plot depicting voltage profile with $S_{1}, S_{5} \& S_{7}$ closed for line faults at 1-2 \&

14-15 with shunt compensation. 74

Figure 5-24: MATLAB plot depicting reconfiguration network for fault at lines 1-2 and 14-

15. 75

Figure 5-25: Plot depicting voltage profile with $S_{1}, S_{5} \& S_{7}$ closed for line faults at 1-2, 14-

15 76

Figure 5-26: MATLAB plot depicting reconfiguration network for fault at lines 1-2, 14-15 and $11-12$ 77

Figure 5-27: MATLAB output command window showing solution of Test Case $5 \ldots \ldots \ldots . . .78$ 
Figure 5-28: MATLAB plot depicting reconfiguration network for fault at lines 9-10 and 10-

11.

Figure 5-29: MATLAB plot depicting reconfiguration network for fault at line $1-2 \ldots \ldots \ldots 81$

Figure 5-30: Plot of Execution Time for Fault Detection Algorithm [2] . ....................... 82

Figure 5-31: Plot of Execution Time for Fault Reconfiguration Algorithm. .................... 82

Figure 5-32: Plot of Execution Time for Fault Reconfiguration Algorithm accessing data

base.

83 


\section{LIST OF TABLES}

Table 1-1: FIPA ACL Message Parameters [14] .............................................. 10

Table 2-1: SWOT matrix for current Agent Applications to power systems [15]. ............ 15

Table 4-1: Description of the notations used in Mathematical model. ........................... 43 


\section{Nomenclature}

$\begin{array}{ll}\text { ACL } & \text { Agent Communication Language } \\ \text { CoF } & \text { Circuit of the Future } \\ \text { DER } & \text { Distributed Energy Resources } \\ \text { DEW } & \text { Distributed Engineering Workstation } \\ \text { DG } & \text { Distributed Generators } \\ \text { FIPA } & \text { Foundation for Intelligent Physical Agents } \\ \text { GUI } & \text { Graphical User Interface } \\ \text { JADE } & \text { Java Agent Development Framework } \\ \text { KQML } & \text { Knowledge Query and Manipulation Language } \\ \text { LAG } & \text { Load Agent } \\ \text { GAG } & \text { Global Agent } \\ \text { MAS } & \text { Multi-agent System } \\ \text { OOP } & \text { Object Oriented Programming } \\ \text { SAG } & \text { Switch Agent } \\ \text { SCE } & \text { Southern California Edison } \\ \text { WVU } & \text { West Virginia University }\end{array}$




\section{Chapter 1}

\section{INTRODUCTION}

\subsection{Background}

The Electrical Power System is the most capital intensive and most complex system ever developed by man. The main era of electrical power system was started by the invention of electric bulb by Thomas Alva Edison in 1879. He also started generating the electricity by building small generating stations. Edison's method of generating and transmitting electricity was called Direct Current (DC) or Low Voltage. The main problem of DC power was that it cannot be transmitted to long distances and cannot be transformed from one voltage level to another. During this time all the loads were supplied at the same voltage level [1].

In 1888, Nikola Tesla invented the alternating current generation. In an Alternating Current (AC) system, transformers were used to step up the voltage levels and enabled the electricity to transfer over long distances. Starting from then many forms of generating the electricity came in to existence like, Thermal Power Generation, Hydro Power Generation, Diesel Power Generation and Gas Power Generations. Development in Nuclear Physics started the nuclear power generation in the recent years and made it a cheaper way of generating power. Because of the technology development even renewable energy sources are making their mark on the power generation. 
Today Distributed Energy Resources (DERs), some times called Distributed Generators (DGs) are provided at the distribution level for serving some important local loads such as, hospital loads. The main reason for providing DER is that it serves as a local generation when there is a shortage of power supply through the grid. Power system is suffering from congestion issues because of deregulation. These issues can be solved by installing DERs near the load centers. However, Interconnection of these DERs is making the system more complicated. After inserting the DERs in to the system, we need to make sure that the system stability and voltage profile are within the safe limits. Even we need to come up with new protection coordination schemes to incorporate these DERs in to the system [2].

The Electric Power System is growing rapidly and becoming more complex each day because of its continuously increasing power demand. Besides the increasing demand deregulation in the power industry is another fact, which is making today's electric power system to be more intrinsic. In the past, the electric power industry has been vertically integrated i.e., a central authority monitored and controlled all the activities in generation, transmission, and distribution. For the last decade or so, the electric power industry has been undergoing a process of restructuring, especially separation of transmission from generation activities. Deregulation is intended to encourage competition among utilities and power marketers to reduce energy prices. There is a need of a power system operator, to coordinate the dispatch of generating units to meet the expected demand of the system across the transmission grid. However this deregulation brought so much of complexity in the electric power system which is needed to be handled carefully [3].

Electric power system is such a vast and sensitive network that even a single fault can cause the whole system to be down. A single fault normally causes another fault in the system and this process keeps on going until the whole system collapses. This particular phenomenon in power system is said to be Cascading Effect. A power system operator, who generally monitors and controls the whole system, has to be very careful regarding this cascading phenomenon. A small human mistake can cause a massive black out in the power network leading to billions of dollar loss. A great example for this type of black out is the 2003 Northeast Blackout [4]. The major reason for the Northeast Blackout was a sudden power fluctuation in a particular area of Ohio. The outage of Ohio generating station triggered the cascading effect, causing several high voltage transmission lines to 
go over their thermal limit. These sagged transmission lines then touched the overgrown trees in Ohio area and caused several ground faults. These things cascaded together and brought down approximately 100 generating stations. This was one of the major blackouts in the power history which caused nearly 50 million people to live in dark and 6 billion dollars of financial losses [4].

The one reason behind this massive havoc was lack of controlling on power grid using EMS (Energy Management Systems) and Restoration techniques available at that time. The power quality and reliability can be improved by having better fault detection and reconfiguration techniques. These can be achieved by a novel technique called Multiagent systems. Multi-agent systems monitor and control the power systems in an autonomous and decentralized manner. They use their communication capability to communicate and negotiate on a particular task. Recent technology developments and drastic reduction of cost are making this Multi-agent system a most viable solution.

\subsection{Multi-Agent Systems}

Agent based technologies have been the subject of extensive research and investigation for several years, but it is recently that these have been seen any significant degree of exploitation in commercial applications. Multi-agent systems have been used in vivid applications, ranging from small systems to most intrinsic systems. Some of the fields where these Multi-agent systems have been fruitfully employed include process control, system diagnostics, manufacturing, transportation logistics and network management [5].

Before understanding the Multi-agent systems one has to understand the concepts behind the single agent. Many people have defined the word 'agent' in their own ways since the starting of agent technologies. Out of many definitions available a couple of relevant definitions are given below

"An agent is an autonomous computational entity such as a software program that can be viewed as perceiving its environment through sensors and acting upon this environment through its effectors" - Weiss [6]. 
"An agent is a computer system that is situated in some environment, and that is capable of autonomous action in this environment in order to meet its design objectives" Wooldridge and Jennings (1995) [7].

The above two definitions are much suitable for this thesis project. Multi-agent systems received greater attention in the recent past in many intelligent, distributed systems to model the theories of interactivity in human societies. Humans interact in various ways and at different levels. For instance, humans observe and model one another, they request and provide information, they negotiate and discuss, they develop shared views of their environment, they detect and resolve conflicts, and they form organizational structures such as teams, committees, and economies [8].

In this thesis the term agent means, a software entity which perceives its environmental changes and acts on it diligently to achieve its organizational objectives. A Multi-agent system means the collection of such agents which communicate and coordinate to solve a particular problem according to their objectives. The Multi-agent system approach for power system is shown as below,

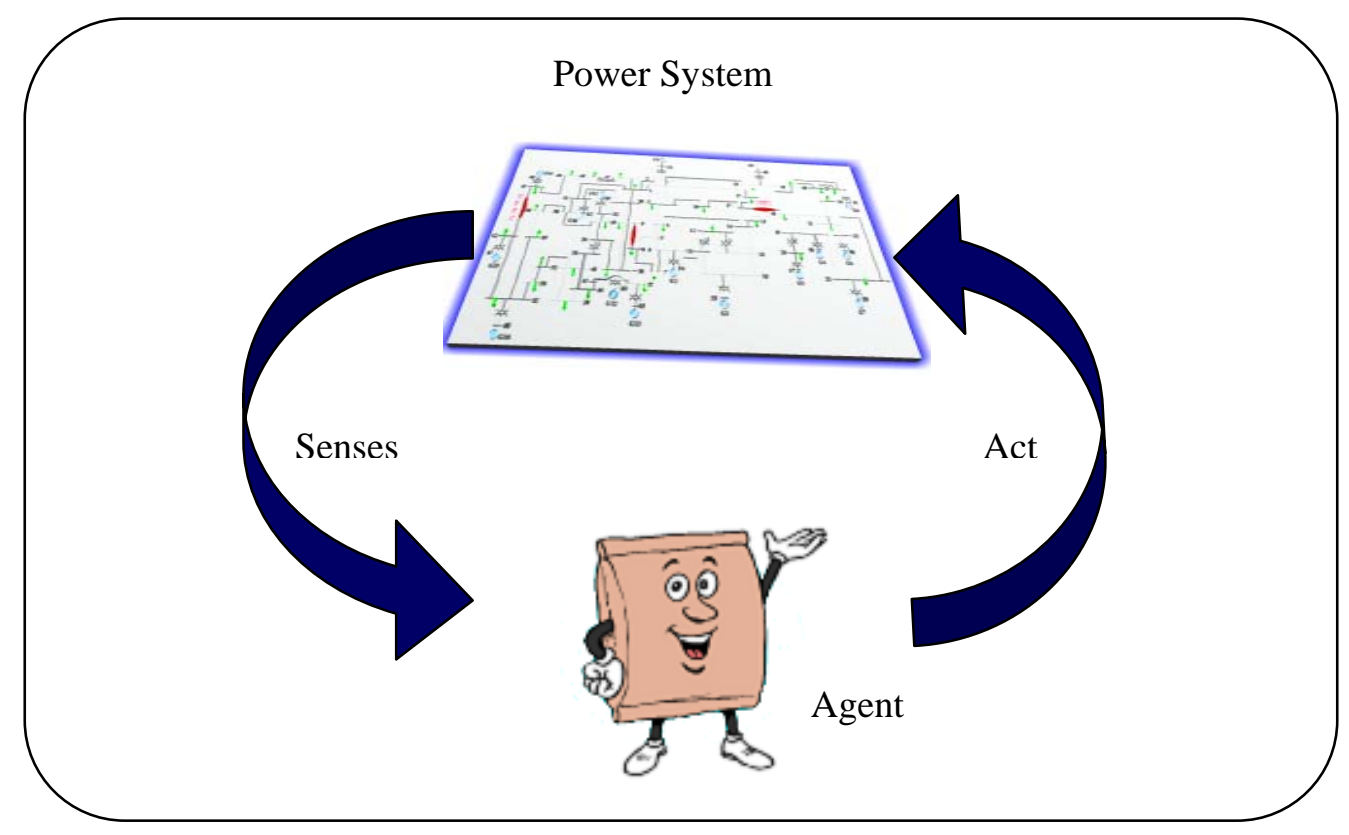

Figure 1-1: A Multi-agent System for Power System. 
The main problem facing by an agent is deciding which of its actions it should perform in order to best satisfy its organizational objectives. The type of decision selected always depends upon the type of environment. Russell and Norvig [9] proposed following classification of environment properties,

- Accessible vs Inaccessible.

An accessible environment is one in which all the states of the environment are easily accessible. But most of the today's complex networks, such as every day physical world and Internet, are inaccessible.

- Deterministic vs Non-deterministic

In deterministic environment the actions will have certain effects on the environment. In non-deterministic environments it is difficult to anticipate the effects of particular action on the environment. Non-deterministic environments will have greater difficulty in designing.

- Episodic vs Non-episodic

In an episodic environment, the performance of an agent is dependent on a number of episodes, such as mail sorting system.

- Static vs Dynamic

A static environment is one that can be assumed to be unchanged except for the actions by agent. In contrast to the static environment the real world is most dynamic one which changes time to time hence, difficult to control.

- Discrete vs Continuous

An environment is said to be discrete, if it has discrete number of actions and percepts in it. The best example for a discrete environment is chess game and for a continuous environment is car driving.

Comparing the agents to the present world computer systems, every action a computer performs is anticipated, planned for, and coded by a programmer. When a computer encounters a new sort of problem, which is not anticipated, then the system will crash. This is because the computer systems don't have intelligence embedded in it. Unlike the computer systems the agents are intelligent enough to take up the flexible autonomous actions to satisfy their objectives [7]. Here the flexibility means three things 
- Reactivity:

Agents are able to perceive their environment, and respond in a timely fashion to changes that occur in it, in order satisfy their objectives.

- Pro-activeness:

Intelligent agents are able to exhibit goal oriented behavior by taking the initiative in order to satisfy their objectives.

- Social-ability:

Intelligent agents are able to communicate and cooperate with other agents available in Multi-agent systems in order to satisfy their objectives.

Out of the above three characteristics the social-ability is more complex one. In the real world daily millions of computers exchange the information with both humans and other computers. But the ability to exchange the bit stream is not really the social ability. Consider the human world, humans achieve their goals by negotiation and cooperation [6]. The same way intelligent agents cooperate and negotiate with each other to achieve the common goal of the Multi-agent system. The communication between the agents in MAS has certain standards defined by FIPA (Foundation for Intelligent Physical Agents).

There is always been a great amount confusion between agents and objects, expert systems. The main distinctions between traditional view of an object and our view of an agent is listed below [6],

- Agent embody a strong notion of autonomy than objects, they decide on themselves whether or not to perform an action on request from other agents.

- Agents are capable of flexible (reactive, pro-active, social) behavior. Where objects have nothing to do with these behaviors.

- A MAS is inherently multi-threaded, each agent has at least one thread of control.

Expert systems are most important Al (Artificial Intelligence) technology of 1980s [10]. An Expert system is one that is capable of solving problems or giving advice in some knowledge rich domain [6] [11]. The main difference between Expert systems and Agent technologies is that, an Expert system cannot sense its environment directly. Instead, it 
needs a middle man to input the data. In the same way an Expert system cannot act directly on its environment. It just gives certain instructions to the operator.

\subsection{Agent Communication}

It has been seen in the earlier section that agents will communicate to coordinate and negotiate on particular task to achieve its organizational objectives. The communication ability of agents is part perception (the receiving of messages) and part action (the sending of messages). There are generally two message types in Agent Communication they are, Assertion and Queries. Every agent, whether active or passive, must have the capability to accept the information. In order to assume a passive role in a dialogue, an agent must be able to 1 ) accept a query from an external source and 2) send a reply to the source by making an assertion. In order to assume an active role in a dialogue, an agent must have the capability to send queries and make assertions [6].

Communication protocols are very important in any communication systems. The Multiagent communication is specified by a data structure with the following five fields,

\section{Sender.}

2. Receiver(s).

3. Language in protocol.

4. Encoding and Decoding functions.

5. Action to be taken by the receiver(s).

Speech Act Theory helps in defining the message send by sender. So, that the receiver has no doubts regarding the received message [12]. In order to understand and to be understood communication messages precisely, all the agents in MAS has to follow the same ontology. Two agents following the same ontology means, those two agents will know the meaning of messages during the communication. Hence, ontology results in perfect operation of communication. In technical terms the name ontology is defined as follows, 
"Ontology is a formal definition of a body of knowledge. The most typical type of ontology used in building agents involves a structural component. Essentially a taxonomy of class and subclass relations coupled with definitions of the relationships between these things" - Jim Hendler [6]

The main languages which are followed for the agent communication are,

- Agent Communication Language (ACL) by the Foundation for Intelligent Physical Agents (FIPA)

- Knowledge Query and Manipulation Language (KQML)

\subsubsection{FIPA-ACL}

Foundation for Intelligent Physical Agents (FIPA) is an IEEE Computer Society Standards organization that promotes agent-based technology and the interoperability of its standards with other technologies. FIPA was originally formed as a Swiss based organization in 1996 to produce software standards specifications for heterogeneous and interacting agents and agent based systems [13].

FIPA has developed a certain number of standards for heterogeneous agent communications. FIPA Agent Communication specifications deal with Agent Communication Language ( $A C L)$ messages, message exchange interaction protocols, speech act theory based communicative acts, and content language representations.

Agent Communication Language (ACL) developed by FIPA is based on speech act theory. Here the messages are actions, or communicative acts, as they are intended to perform some action by virtue of being sent. The FIPA ACL specifications consist of a set of message types and the description of their pragmatics, which is the effect on the mental attitudes of sender and receiver agents. A sample FIPA ACL message is as shown below, 


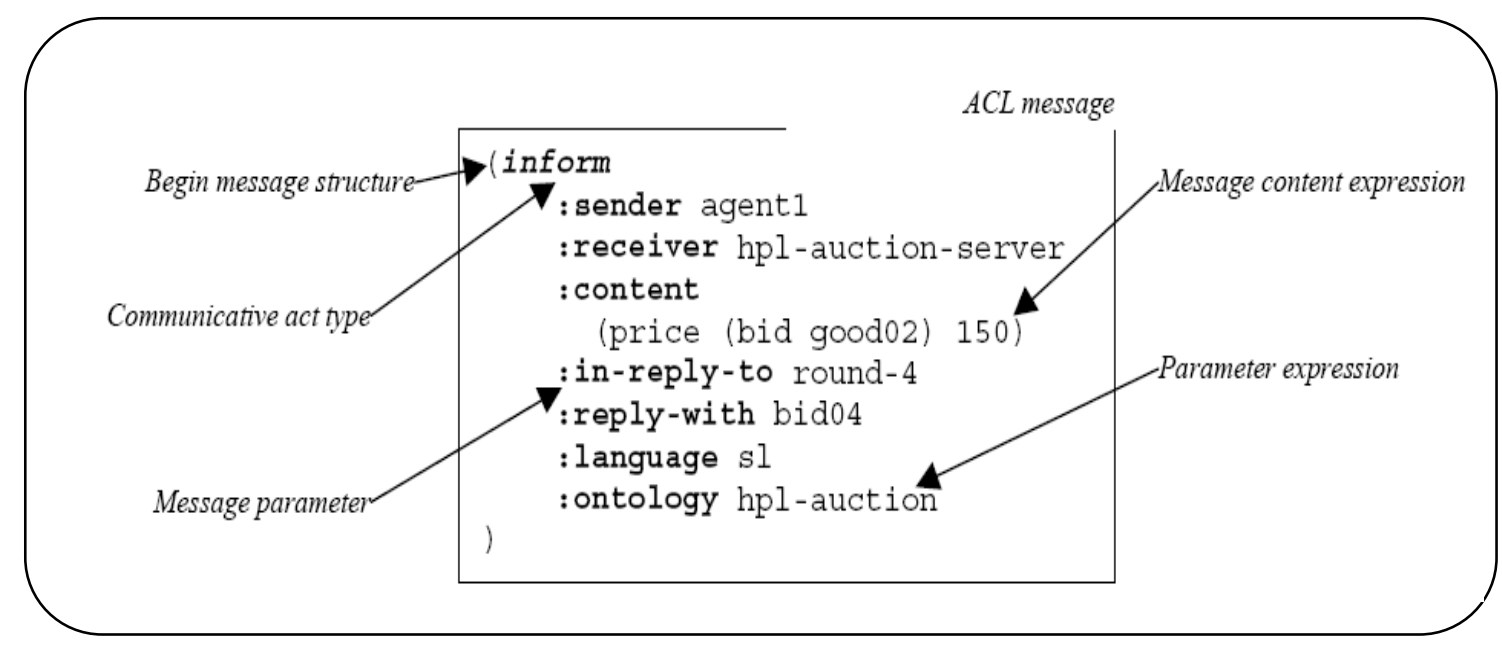

Figure 1-2: FIPA ACL message [14].

As noted above, the message contains many parameters. These parameters can occur in any order in the message. The only parameter which is mandatory for the agent communication is :receiver parameter. However, for a meaningful message there should be other parameters required depending upon the situation. The full set of pre-defined message parameters is shown in the following table.

\begin{tabular}{|l|l|}
\hline $\begin{array}{l}\text { Message } \\
\text { Parameter }\end{array}$ & Meaning: \\
\hline :sender & $\begin{array}{l}\text { Denotes the identity of the sender of the message. i.e., the name of the } \\
\text { agent. }\end{array}$ \\
\hline :receiver & Denotes the identity of the intended recipient of the message. \\
\hline :content & $\begin{array}{l}\text { Denotes content of the message; equivalently denotes the object of the } \\
\text { action. }\end{array}$ \\
\hline :reply-with & $\begin{array}{l}\text { An expression which will be used by the agent responding to this } \\
\text { message to identify the original message. Can be used to follow a } \\
\text { Conversation thread in a situation where multiple dialogues occur } \\
\text { simultaneously. }\end{array}$ \\
\hline :in-reply-to & $\begin{array}{l}\text { Denotes an expression that references an earlier action to which this } \\
\text { message is a reply. }\end{array}$ \\
\hline :envelope & \begin{tabular}{l} 
Denotes an expression that provides useful information about the \\
\hline
\end{tabular}
\end{tabular}




\begin{tabular}{|l|l|}
\hline & $\begin{array}{l}\text { message as seen by the message transport service. The content of this } \\
\text { parameter is not defined in the specification, but may include time sent, } \\
\text { time received, route, etc. }\end{array}$ \\
\hline :language & Denotes the encoding scheme of the content of the action. \\
\hline :ontology & $\begin{array}{l}\text { Denotes the ontology which is used to give a meaning to the symbols } \\
\text { in the content expression. }\end{array}$ \\
\hline :protocol & $\begin{array}{l}\text { Denotes a time and/or date expression which indicates a guideline on } \\
\text { the latest time by which the sending agent would like a reply. }\end{array}$ \\
\hline id & $\begin{array}{l}\text { Introduces an identifier which denotes the protocol which the sending } \\
\text { the interpretation of the message. }\end{array}$ \\
\hline iconversation- & $\begin{array}{l}\text { Introduces an expression which is used to identify an ongoing } \\
\text { sequence of communicative acts which together form a conversation. A } \\
\text { Conversation may be used by an agent to manage its communication } \\
\text { strategies and activities. }\end{array}$ \\
\hline
\end{tabular}

Table 1-1: FIPA ACL Message Parameters [14].

The ACL language has been used in JADE, a Java agent based platform developed by Telecom-Italia.

\subsubsection{Knowledge Query and Manipulation Language (KQML)}

Knowledge Query and Manipulation Language (KQML) is older than the FIPA ACL language. The Knowledge Query and Manipulation Language (KQML) is a protocol for exchanging information and knowledge. The beauty of KQML language is that, all information for understanding the content of the message is included in the message pattern itself [6]. The basic protocol for the KQML language is defined by the following structure, 
(KQML-performative

$$
\begin{array}{ll}
\text { : sender } & <\text { word }> \\
\text { : receiver } & <\text { word }> \\
\text { : language } & <\text { word }> \\
\text { : ontology } & <\text { word }> \\
\text { : content } & <\text { expression }> \\
\text {..) } &
\end{array}
$$

The KQML protocol for communications among both agents and application program is illustrated in the following figure,

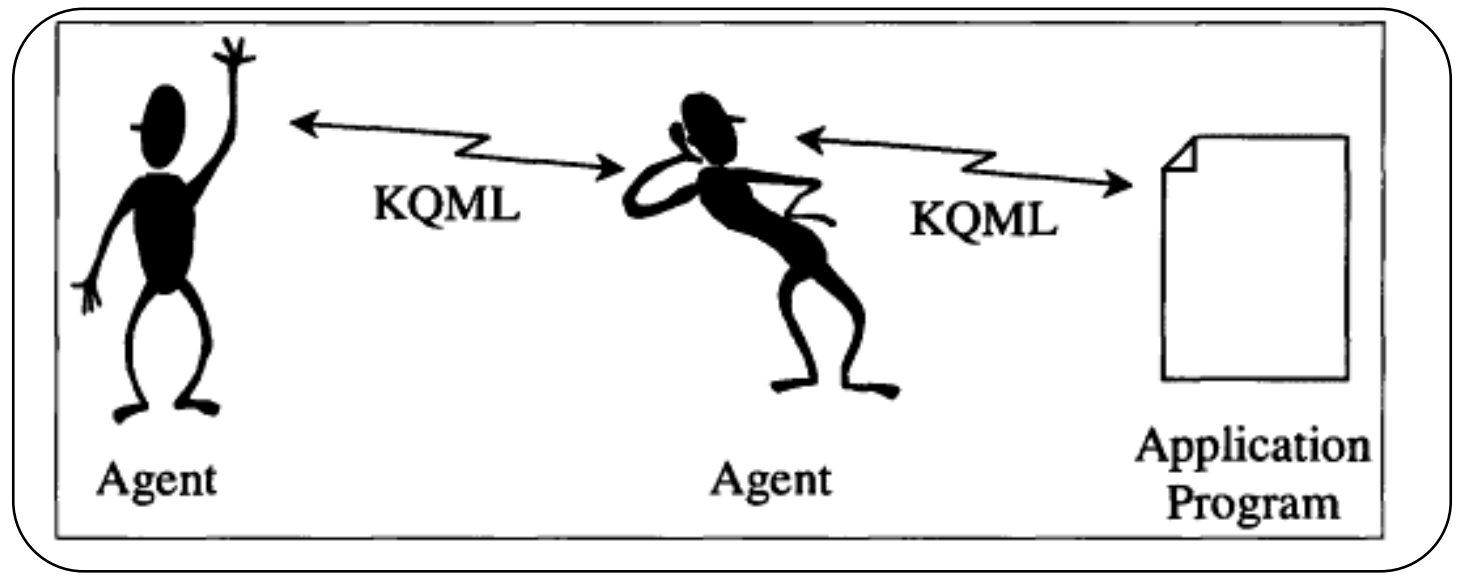

Figure 1-3: KQML communication protocol [6].

Most of the today's agent based platforms are migrating to FIPA ACL language for the agent communications.

\subsection{Problem Statement}

In this thesis an agent based platform is developed for a distribution system. This Agent platform will serve as an automatic fault locator and reconfiguration entity for the distribution system. The study is based on a proto-type circuit, the Circuit of the Future (CoF) developed by Southern California Edison (SCE). 
An agent is located at each node, load, source and switch in the distribution system. When a fault occurs in the network, agents will start communicating to each other and come up with the fault location. Once the fault is detected depending upon the fault, the reconfiguration algorithm comes up with reconfigured network in which all the loads will get supplied. The final step in the reconfiguration is to do the voltage control by using shunt compensation and priority based load shedding.

A few assumptions made in this thesis work are listed below,

- The substation bus is modeled as an infinite bus. Assuming it is connected to the power-grid, where it can pull up the required power.

- DG is modeled as a backup source.

- When a fault is identified, the protection is applied first and isolates the faulty lines, before reconfiguration

\subsection{Approach}

The original CoF, is simplified by lumping certain loads without affecting the switch locations and maintaining the original topology of the system. The circuit is modeled using Distributed Engineering Workstation (DEW), power distribution software developed by Electrical Distribution Design Inc. Power World Simulator is used for single phase simulation of circuit of the future network. The software used to design the MAS is Java Agent Development (JADE) framework and MATLAB. Fault detection algorithm is developed by JADE and reconfiguration algorithm is implemented in MATLAB. This software will be presented in detail in Chapter 3.

The agent frame work in JADE will first detect the fault location and the global agent, which has the capability of performing load flow analysis on the circuit, will use the MATLAB reconfiguration algorithm to reconfigure the circuit. The one solution with less power losses and good voltage profile will be chosen as the final solution, when multiple solutions are available for the reconfiguration. In the Reconfiguration algorithm the voltage control is achieved by shunt compensation and priority based load shedding. 


\subsection{Outline}

The outline of the remaining chapters is given in this section.

Chapter 2 will describe the literature overview of different multi-agent applications in power distribution systems. It also covers a few other applications of multi-agents outside the power engineering field.

A detailed description of the software packages used in this thesis is given in Chapter 3. The advantages of this software and the suitability to the relevant applications are also discussed.

A mathematical model, based on graph theory, for fault detection and reconfiguration algorithms will be presented in Chapter 4. This chapter will include the modeling of the system and the Circuit of the Future that is used in this work to perform the simulations.

Chapter 5 will present the simulations and results of the model. Finally, conclusion of this study and the future work is detailed in Chapter 6 . 


\section{Chapter 2}

\section{LITERATURE REVIEW}

This chapter deals with comprehensive overview of the Multi-agent applications in power system field and many other engineering disciplines. The chapter starts with the explanation of Multi-agent system technology and its overview. Applications of Multi-agent systems in power systems, such as in automatic fault detection, reconfiguration, protection coordination, voltage control, reactive power control and power markets are presented in detail manner in the later section of the chapter. Finally the chapter concludes with explaining the Multi-agent system advantages over the traditional schemes available and technical difficulties of Multi-agent systems.

\subsection{Multi-agent systems}

A Multi-agent system is a computational system in which several agents cooperate and coordinate with each other to achieve some objectives. Multi-agent systems have the capability to solve problems which are difficult or sometimes impossible to solve by an individual agent. Multi-agents systems are a branch of Distribute Artificial Intelligence (DAl). As a social community the agents are able to evolve, self produce, learn, cooperate, and adapt. According to [6] the Multi-agent system environment has the following characteristics, 
- Multi-agent environment provide an infrastructure specifying communication and interaction protocols.

- Multi-agent environments are highly decentralized structures.

- Multi-agent environments contain agents that are autonomous and distributed, and may be self-interested and cooperative.

Research on Multi-agent systems is still an ongoing process. Although Multi-agent systems have shown good results, these technologies are yet to make a significant impact in the power system area. The authors in [15] have analyzed agent based technologies under the Strengths Weaknesses Opportunities Threat (SWOT) framework. Based on the SWOT analysis they have envisaged the future application of agents in the power system area. The following table shows the SWOT matrix developed by them

\begin{tabular}{|c|c|}
\hline Strengths & Opportunities \\
\hline $\begin{array}{l}\text { - } \quad \text { No single point of failure } \\
\text { - Decentralized data processing } \\
\text {-Fast operation } \\
\text {-Task distribution } \\
\text { - Ease of tackling complex problems } \\
\text { - Natural solution } \\
\text { - Leads to scalable, modular and } \\
\text { flexible solutions }\end{array}$ & $\begin{array}{l}\text { - } \text { Technological advances } \\
\text { - Collaboration/cooperation } \\
\text { - Develop generic agents/MAS } \\
\text { - Existing hardware or software } \\
\text { infrastructure. } \\
\text { - Learn from other distributed } \\
\text { technologies. }\end{array}$ \\
\hline \begin{tabular}{ll}
\multicolumn{1}{c}{ Weaknesses } \\
- & Can lead to unstable operation \\
- & Complexity \\
- & Lack of understanding \\
- & Lack of experts \\
- & Still its in infancy \\
- & Lack of practical application in power \\
& area
\end{tabular} & 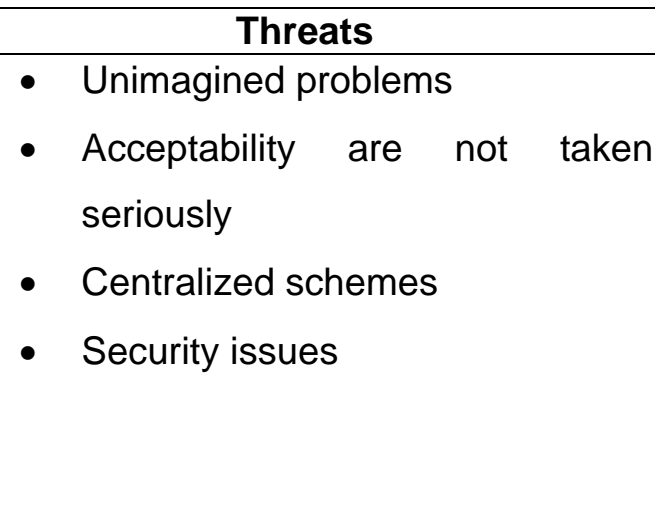 \\
\hline
\end{tabular}

Table 2-1: SWOT matrix for current Agent Applications to power systems [15]. 
Z. Zhang et al. in [16] identified and described three major problems of today's power system industry. They are distributed computing, communications, and data integration. They have suggested that the Multi-agent system technology would be a best solution for the above problems in power systems because of the power system's distributed nature. They illustrated that the MAS can offer modular, flexible, and integrated approach for many problems in the power system area. They have proposed a four stage (Analysis, Design, Implementation, and Deployment) problem solving mechanism using Multi-agent system.

\subsection{MAS in Automatic Fault Detection and Reconfiguration}

Fault detection is a very important thing in providing survivability, reliability, availability, and efficiency of the power system. Many authors have contributed in the field of automatic fault reconfiguration and restoration of the power system.

Authors L. Liu et al. in [17] have proposed a new agent paradigm for fault detection and prognosis in an electric war ship. They have described the inability of traditional diagnosis schemes over the new software agent technologies for fault detection. Prognosis is the important thing in their work, where they can predict the future conditions in a time ahead and can plan for the future actions to avoid them. The proposed agent architecture has two types of agents named diagnosis agents and prognosis agents. A test case on permanent magnet synchronous motor (PMSM) has been demonstrated and the results were promising.

In [18] and [19], the authors have described the automatic fault detection and fault diagnosis (FDD) mechanism using model based methods. These methods are based, e.g., on parameter estimation, parity equations or state observers. The main aim of their work is to create several symptoms to identify the difference between normal and faulty systems. The fault in the system is detected by the use of classification or interference methods. Authors in [20] and [21] proposed the fault diagnosis and detection mechanism using analytical redundancy. In [22] K. Huang et al. had given a brief survey about the agent technologies available for fault detection and restoration in Ship board Power 
System (SPS). They presented a decentralized scheme of agent technology for automatic fault detection. Several test cases have been demonstrated to prove the proposed work. In [23], the authors presented different online techniques for fault detection.

Several authors have presented new methods of fault reconfiguration schemes since a decade. New technologies like Agent technologies are getting more emphasis in the field of fault detection and restoration of power systems. Many authors described the advantages of decentralized schemes over the centralized schemes.

In, [24] the authors proposed MAS for power distribution system. Unlike the traditional centralized reconfiguration schemes, they proposed a completely new decentralized reconfiguration scheme. In their Multi-agent systems each agent represents a major component in the power system such as generators, breakers, and loads. Each agent will communicate to its neighboring agent only, which makes the whole agent system more decentralized. The agent platform is created in Java Agent Development Framework (JADE), and the real power system is implemented in a real time digital simulator. The agents are implemented in iPAQs, iPAQ is a Pocket PC (PPC) developed by Hewlett Packard. Power system is modeled in Real Time Digital Simulator (RTDS). The series ports available in RTDS are used for the communication between RTDS and iPAQs. However, an interface named Field Programmable Array Gate (FPGA) is developed for the communication because of the voltage difference between RTDS and iPAQs.

In [25], the authors proposed a Multi-agent system approach for reconfiguration and restoration of Electric warship. The proposed scheme is a decentralized one, which has advantage of avoiding the single point failure as of in centralized reconfiguration scheme. In their work they have introduced three kinds of agents, named Switch Agents (SAs), Substation Breaker Agents (SBAs) and Tie Breaker Agents (TBAs). Fault detection in the system is based on monitoring the fault currents of neighboring agents. The ship board power system is modeled using three phase unbalanced PQ load models. Ship board system is developed in Virtual Test Bed (VTB) and the agent platform is developed in Matlab.

In [26], the same authors came up with a better agent model for the power system reconfiguration and restoration problem. The Multi-agent platform is developed in Java 
Agent Development Framework (JADE) and the power system test case is developed in Virtual Test Bed (VTB). In their work they proposed three new agents named Switch Agents (SAs), Load Agents (LAs), and Generator Agents (GAs). Here the agents communicate only to their neighboring agents and act locally, making the restoration decentralized. The restoration algorithm has certain objectives and constraints like, limit on generation, priority of loads, and transfer capacity of lines. The three phase unbalanced load flow is carried out by forward and backward propagation.

Takeshi Nagata et al. [27] have greatly contributed in the area of MAS for the restoration of distribution systems. They have proposed a restoration method by joint usage of Expert Systems (ES) and a Mathematical Programming (MP) approach. The main purpose of their work is to determine the optimal target system for restoration using Mathematical Programming (MP). They introduced a new topic called "restorative operation cost", which has decreased the number of rules in the knowledge based Expert Systems (ESs). The Expert systems available at that time were inefficient in finding the optimal target configuration. The authors took up the same knowledge based Expert Systems and used Mathematical Programming methods to improve their capability of finding the optimal target configuration. Several test scenarios are presented in their work to demonstrate the combined technology of Expert Systems and Mathematical Programming.

The same authors in [28], [29] have proposed a Multi-agent architecture for power system restoration problem. This architecture was fully decentralized one, in which the agents communicate and negotiate only with their neighboring agents. In this work authors have used two types of agents named Bus Agents (BAGs) and Facilitator Agent (FAG). BAG is a bus agent situated at each and every bus in the network whereas, FAG is a Facilitator Agent situated on top of all BAGs for the decision making purpose. BAGs communicate with their neighboring agents to find out the suboptimal target configurations where as, FAG acts as a manager for decision process. Knowledge Query and Manipulation Language (KQML) has been used as an agent communication language.

In [30], authors Takeshi Nagata et al. proposed new agent architecture for power distribution system. In this publication the proposed method has a new agent called Junction Agent (JAG). JAG is a Junction Agent situated at each and every multi terminal transmission line to monitor and control it. The authors have used Foundation for 
Intelligent Physical Agents (FIPA) compliant XML/ACL, which is ACL (Agent Communication Language), mounted on XML (Extensible Markup Language) for communication between the agents. Several test scenarios are presented in their work demonstrate the proposed algorithm for the reconfiguration and they sound promising for the large scale power system restoration.

Later the same authors in [31] have proposed a decentralized Multi-agent architecture for the bulk power system restoration. In this work they have introduced hierarchy among the agents. They classified the agents into upper and lower level agents. The proposed system consists of several Local-area Management Agents (LMAs) and Remote-area Management Agents (RMAs). LMAs and RMAs are located in the upper level for monitoring and control purposes. Several Load Agents (LAGs) and Generator agents (GAGs) are situated in the lower level of physical power system network. LMAs are responsible for the local area restoration by means of communicating and negotiating with the neighboring LMAs. RMAs facilitate the restoration of loads within the remote area. LAGs and GAGs correspond to load management system and generator management system which store their respective loads and generators local information. In addition to their local communication, they also interact with the upper level agents.

In [32], authors have proposed a more complicated four layered agent architecture for the bulk power system restoration. A single Independent System Operator Agent (ISOAG) which controls the timing of the simulation process forms the higher level in the agent architecture. The second level consist several Local Area Management Agents (LAMs) for the local area management for the restoration. Third level of architecture has several Load Facilitator Agents (LFAGs), Generator Facilitator Agents (GFAGs), and Remote Facilitator Agents (RFAGs). These agents will facilitate with the agents situated in the fourth layer of architecture which are, Load Agents (LAGs) and Generator agents (GAGs). The authors have applied the proposed MAS to bulk power system and the simulation results are very promising.

Authors in [33] have also contributed in the field of Multi-agent system for bulk power system restoration. The method employed in this work makes use of both centralized and decentralized schemes to obtain the restoration solution. The agent architecture has two hierarchical agents called, Management Agents (MGAGs) and Agents representing 
practical power system components (PCAGs). Each PCAG monitors several agents related to a subsystem named Generator Agents (GAGs), Substation Agent (SAGs), and Load Agents (LAGs). One MGAG is generated to in charge of the subsystem and provides negotiations and communications between PCAGs and with other subsystems.

\subsection{MAS in Distribution System with DER}

The power grid has been changing its physical infrastructure, control and communication infrastructures because of the deregulation in the power industry. Many distributed generation firms with less pollution and cheap operating costs came into the field of generation, making the energy market competitive. Distributed Energy Resources (DERs) can improve the reliability, stability, and quality of the electric grid. However, distributed energy resources bring several problems related to connection to the electric grid and the protection issues.

In [34], authors have proposed a hybrid Multi-agent system to achieve scalability for control of a large network of power generation, transmission, load, and compensation sources. Several ancillary agents are developed for system stability and harmonic and reactive current compensation in the paper. Scalability is the greatest issue present in huge networks such as power network, where the power network has thousands to tens of thousands nodes. In decentralized architecture agents makes use of local information to achieve their goals. The information could be values of voltages, currents sometimes may be the power values. Authors have presented a hybrid agent architecture where agents can connect not only to their parents and children but also to their siblings. Peer agents can communicate and negotiate with each other. Peer agents will dynamically select a leader to establish the real connection with their parents. In the proposed agent architecture, authors have developed several compensator agents to take care of power electronic compensators in the power system. The power electronic compensator has several responsibilities such as reactive power generation, power flow control, harmonic compensation, voltage regulation or dynamic control over the frequency and voltage. A large set of decision criteria is necessary for the agents such that they can make control decisions that will ultimately improve the power quality and reliability of the electric grid. 
T. Hiyama et al. in [35] presented a Multi-agent based operation and control of distribution system with dispersed power sources such as a photo-voltaic unit, a wind generation unit, a diesel generation unit, and an Energy Capacitor System (ECS). Several simulation studies have been performed to prove that the proposed methods have increased the control performance even in the existence of communication delays.

In [36] the same authors proposed a reconfiguration technique using the Multi-agent technology for the distribution system which has several kinds of distributed generation. The distributed generation includes Diesel Generator (DG), wing generator, photo-voltaic and ECS. ECS is an energy storage device and plays a main role in load following operation. Computer networks are used as communication channels for communicating the information about DGs and ECSs. Authors have introduced a coordination scheme between diesel unit and ECS with the help of multi agents. Reconfiguration has been done both for radial and mesh networks.

\subsection{MAS in protection coordination}

Many in the research community have conducted research concerning to the MAS application for the protection coordination in power system. It is very important task for agents in the protection coordination in order to ensure power system safety and reliability. Selectivity, Celerity, Sensitivity, Reliability in relaying protection have already been improved, but the system is lack of cooperation and coordination to solve the problems. Relays play a vital role in power system operations with very low tolerance for failure. Hence, MAS technology can be a promising solution for all of these protection coordination problems in power network.

In [37], the authors have proposed Multi-agent system approach for the post-fault disturbance diagnostics. Protection engineers use data from different monitoring devices to perform the post-fault disturbance diagnostics. In the past, several heterogeneous intelligent systems have been developed to interpret the data received and to assist the engineers in post-fault disturbance diagnostics. Majority of the system were standalone 
because of the system integration issues. In this paper a novel MAS architecture has been developed named protection engineering diagnostic agents (PEDAs). The new proposed method delivers flexibility and scalability features. These agents will integrate Supervisory Control and Data Acquisition (SCADA) systems with new systems for Digital Fault Recorder (DFR) for enhancing the fault record retrieval from remote DFRs. The paper elaborates the benefits of a MAS approach and design and implementation of PEDA.

Jinai Zhang et al. in [38] came up with new Multi-agent system architecture for relaying protection in power system. The proposed scheme has a coordination layer where all the agents, which are monitoring the protection in the power network, are coordinating for protection and control of power grid independently and collectively. Authors have used the PEDA technology, discussed in [37], to remove failures correctly and quickly. They have mentioned several advantages of MAS over traditional methods, such as enhancing intelligence, flexibility, reliability of the power system. In [39], the authors have proposed an agent based methodology for current differential relay to use with a utility intranet. Agents, as proposed in this paper are software pieces capable of searching for information in the networks, interacting with pieces of equipment and performing tasks on behalf of their owners (relays). Results have illustrated that the proposed scheme was very promising agent-based differential method acting within a communication structure i.e., intranet.

In [40] the authors have presented an agent based power protection system using supervisors. In this paper the framework of supervisory control of discrete event system (DESs) is applied for agent-based power protection system for achieving high reliability and selectivity. Reliability is a measure of the degree that the protection system will perform correctly when required, and the selectivity is an ability to recognize the fault and to trip the circuit breaker to isolate that fault. The proposed system uses the feedback information of events to achieve its objective goals. A detailed design procedure of a supervisor that coordinates the behavior of relay agents to isolate fault areas through the minimum operations of circuit breakers is presented in this paper.

A Multi-agent architecture is developed in [41] for the protection coordination of power system having distributed generation in it. Protection of power system, which includes 
DGs is going to be more complicated than simple radial network protection. The proposed scheme is capable of such type of protection, High Impedance Fault (HIF) detection, fault location and load shedding for the DG system. The complete design and implementation of relay agents is presented in this paper. The relay agents will coordinate and communicate with other relay agents for performing tasks of protection with autonomy. EMTP simulation results prove that the proposed scheme is very promising MAS for distributed generation.

The authors of [42] have presented a Multi-agent approach for protection coordination with distributed generation in industrial power distribution system. In the proposed system communication will play a major role besides relay settings. The agent architecture is developed using Java Agent Development Framework (JADE). In this work the author proposed three types of agents named relay agent, equipment agent and distributed generation agent. The relay agent is responsible for searching the relevant information by communication, detecting malfunctions of relay, breaker failures and DG connection status. The equipment agent collects the local power system information and acts on the local power system. The distributed agent will communicate with the relay agent to provide the connection status of its own for protection coordination. In [43], the same authors have proposed MAS architecture for substation protection with distributed generation. In this architecture each substation is viewed as one JDE container. Each JADE container again has number of agents in it. In this work author introduced new agent called Substation Management Agent (SMAG). The results presented in the paper are very promising.

The same work on protection coordination is investigated in [44], [45] and [46]. Finally, MAS proves to be a prominent solution for today's protection coordination issues in power system. 


\subsection{MAS in Voltage Control}

Many authors have contributed in the field of voltage control and reactive power control of power system. In this section some of their work is illustrated. Power quality refers to two quantities which are voltage and frequency. Frequency is the measure of balance between the generation and load, and maintained always at nominal value for the power system reliability. Voltage is second quantity which is always kept under certain limits and is the major concern in the power distribution system. MAS technology is a very promising solution for reactive power management and voltage control in power distribution system because of agents supreme attributes such as autonomy, cooperation, Intelligence, adaptation and social behavior.

In [47], the authors have proposed a decentralized agent mechanism for the secondary voltage control in power-system contingencies. The secondary voltage control mainly applied for the generator Automatic Voltage Regulators (AVRs) to improve the power system voltage stability. In this paper, different types of voltage controllers, such as an Automatic Voltage Regulator (AVR), a Static Var Compensator (SVC) or Static Synchronous Compensator (STATCOM), is treated as an agent. The agents which are electrically close are responsible for their own area voltage control. When an agent senses voltage violation it activates its reactive power reserve. If the voltage violation is not cleared by its own reactive power reserve then the agent starts asking reactive power from its neighbor agents. The agents with sufficient reactive power will respond to the respective agent for voltage support. The agents share their common goal and achieve goal by communication and coordination. The proposed scheme is applied on simple power system and the results are promising.

The authors in [48] have presented optimal coordination work for Multi-agent based secondary voltage control in power system. The agent architecture has a set of execution agents and a coordination agent. Individual voltage controllers, such as an AVR, a SVC and a STATCOM represent an execution agent. Under normal conditions, Multi-agent system based voltage co-ordination works as a conventional secondary voltage control and supports Var/Voltage control. When system runs in to contingencies, the Multi-agent system uses contract net protocol to realize coordination and cooperation among voltage control agents for eliminating voltage violation. During the contingency situations the 
execution agents (EA) will act independently and try to restore the voltage in their areas. If the execution agents fail in restoring the voltage within limits, they send message to coordination agent (CA). The coordination agent then will coordinate with its execution agents to restore the voltage profile. Authors have used New England 39-bus system as a test system to demonstrate the proposed agent architecture and the results were up to the mark.

\subsection{MAS in Power Markets}

Power Market is the other major area in the power system, where Multi-agent systems are exploited to a wide range. In [49], the authors have proposed a Multi-agent system for power markets, which makes use of game theory principles. They have developed specialized intelligent software agents that perform negotiations on behalf of their human counterparts, and then suggest the market strategies that the human can adopt. The game theory principles are used for negotiation protocol. The agent model required not to exchange any sort of trustworthy important information between coalition partners. This is the fact where game theory differs from the proposed work in this paper. In [50], the same authors applied this work to a three player market model. This particular model has three generation companies where each individual company wants to maximize its profits.

Hyungna Oh et al. in [51] have investigated about, why price spikes occur in deregulated wholesale market and how efficiently they can be mitigated. The authors have presented MAS architecture to replicate a spot market. The proposed agent architecture has six autonomous adaptive agents representing six generating stations and one Independent System Operator agent representing an Independent System Operator (ISO). The firm learns about the market and its competitor's behavior by comparing actual market outcomes with predicted outcomes based on an estimate of their own residual demand curve. The estimated residual demand curve is updated each interval using a kalman filter. In [52], the same authors presented a agent based work to test how spot prices affected by forward contracts. 
Leigh Testfation et al. in [53] developed a Java agent based whole sale power market simulator. The simulator is referred as Agent-based Modeling of Electricity Systems (AMES) and is designed in accordance with core Federal Energy Regulatory Commission's (FERC)'s recommended design features and operating over a realistically rendered transmission grid. This framework has several learning mechanisms through which generation companies can maximize their profits and utilization factors. Authors have taken a 5-bus test case to illustrate the simulator features.

Several other papers such as [54], [55], and [56] also contributed for Multi-agent system application in power markets.

\subsection{MAS in other engineering disciplines}

Distributed Artificial Intelligence (DAI) is very promising and developing technology in many fields of engineering. Many engineering applications which needs monitor and control aspects are opting for Multi-agent system technology, which is the part of DAI. Several firms in engineering today are exploiting the Multi-agent technology to harness its strengths towards technical problem solving. A few of them are discussed in brief below.

Highway Traffic Management is one area where researchers are using agent based technologies for making them effective and reliable. The traffic congestion in peak hours results in loss of productivity by conveyors, waste of valuable time, increase in accidents, and a risk of public health with the increasing pollution from the vehicles. In [57], the authors have proposed a multi agent system for traffic management which can mitigate the traffic congestion in Automated Highway System (AHS). Autonomous agents cooperate and coordinate with each other to solve problem, such as conflicts, incidents and synchronization. The behavior of agent is described by colored petri nets.

Due to ever increasing intensity of air traffic and its safety issues, MAS proves to be promising solution for Air Traffic Control (ATC). In [58] authors have proposed a agent architecture for ATC which employs several agents playing roles of assistant aircraft crews and assistant air traffic control operator of approach zone. ATC operator agent 
(ATCO) is responsible for coordination of airliner's entries in to the ATC zone. Its knowledge base $(\mathrm{KB})$ consists of rule set representing temporal constraints on concurrent usage of approach schemes. Airliner agent is responsible for control over airline trajectory along assigned arrival scheme. The authors developed a software prototype intended for validation of the Multi-agent model of ATC.

Mine detection is the other specific area where distributed artificial Intelligence techniques such as MAS are widely used. In [59], the authors have studied and implemented hybrid architecture to implement mine detection, obstacle avoidance and route planning by using MAS. There are different techniques to design the mine exploration. The authors have followed frontier based exploration. In this type of exploration the agent builds its own map and starts searching on it. After the coordination mechanism post exploration route planning is carried out. This will enable the agent to generate safe route plans for the given target destinations.

There are several other fields in which MAS technologies have been used for variety of reasons. For example, computer games, logistics, robotics, graphics and e-commerce are some of the fields where extensive research is going on for embedding the Multi-agent systems.

\subsection{Limitations and Technical challenges of MAS}

It is recently that Multi-agent technologies are shifting from laboratory to utility areas to harness its potential features. As stated earlier in the chapter, Multi-agent systems are used in many power systems and many other engineering areas.

Despite the advantages of Multi-agent systems and its growing awareness, some basic questions often arise from the researchers and industrialists, in particular, industrial partners when discussing Multi-agent systems and their role in power engineering. The basic questions are as follows, 
- What are the benefits offered by MAS over traditional technologies?

- What are the major differences between MAS and traditional technologies?

- To which sort of problems MAS can be applied?

These are the key questions regarding to the Multi-agent systems and often confusing. Many researchers have struggled answering these questions. D. J. McArthur et al. in [60] were able to answer the above questions. MAS can encapsulate a particular task or set of functionalities, and acts in a similar way to object oriented programming. In fact MAS is adding another level of abstraction to object oriented programming, not only the internal data structures are hidden but also action of agents (methods) can be hidden. This particular fact makes MAS pioneer in energy markets, where certain things need to be kept in secret between agents. According to [60], the main advantages of MAS are as follows,

- Agent autonomy and agent encapsulation

- Open Multi-agent system architecture.

- Can be used as a platform for distributed systems

- Fault tolerance

The key difference between MAS and its counterparts such as Al techniques, Expert systems, Model based reasoning (MBR) systems, and Artificial Neural Networks (ANNs) is the notion of autonomy and interaction protocols available in MAS. MAS offer a great amount of autonomy feature to its agents, where agents can independently decide on actions to achieve their goals. Another great feature of MAS is that, it has a richer set of interaction protocols which supports negotiations. The authors in [60] have explained clearly that, this MAS technology can be applied to any engineering discipline which requires a decentralized problem solving mechanism. In spite of many advantages of MAS, there are certain technical challenges that are yet to be overcome to allow most effective implementation of multi-agent systems in the power engineering community. The technical challenges are listed below 
- Platforms

Today in the market several Multi-agent platforms are available. A judicious selection of agent platform is required to ensure the long-term compatibility and required robustness for online applications.

- Toolkits

Reuse of existing agent behaviors and capabilities is an important task. Thus, selection of toolkits to perform these activities is a major thing for the benefit of the whole community.

- Intelligent agent design

Standardized agent design architecture is needed for educating the new researchers and industrial implementers about MAS.

- Agent communication languages and ontologies

- Specific data standards

- Security issues

- Mobile agents

Many researchers are interested in moving or mobile agents. These agents actually move from one point to another point in the network. As of now no credible reason for using this approach is apparent.

These above listed things are few technical challenges which have to be overcome such that MAS technologies are widely used in power engineering area. Beyond above technical challenges and implementation issues, the lack of experience in the use of multiagent system technology in the industry is an obvious concern of both utilities and manufacturers. In [61], the same authors stressed on design and implementation issues of Multi-agent systems.

The other main challenge would be interfacing the power system software with the agent platform. Normally the agent platforms are created in some Java based systems, where as power system models are created using traditional power system software. Many times there exists a big difficulty in interfacing these two platforms. In [62], the authors proposed a methodology to interface the MAS application, JADE to the power system application, which is developed in Virtual Test Bed (VTB). The authors have realized this interface through a communication mechanism called Common Object Request Broker Architecture (CORBA). 


\section{Chapter 3}

\section{SIMULATION SOFTWARE}

This chapter briefly describes about various software packages used in this thesis work for modeling the agent-based power distribution system. It is clear from the previous chapter that MAS greatly depend on software simulations and it is very important to choose suitable software packages for the agent based simulations.

Among several technical challenges listed in the previous chapter, selecting a appropriate software package for simulating MAS is the major technical challenge. Several options are analyzed for simulating both power distribution system and Multi Agent System. Finally, MATLAB, MATPOWER, Power World Simulator, Distributed Engineering Workstation (DEW), and Java Agent Development Framework (JADE) are chosen to simulate the power distribution system and agent model in this thesis work.

The whole Multi-agent system model interfaced to power distribution system is depicted in the following figure. The bottom layer in the figure represents physical network of power distribution system. Agents are situated at all components of power system such as, buses, loads, distributed generators, etc. These agents form upper layer in the architecture. There is a great need of an interfacing tool for interfacing the distribution system model to the agent architecture. 


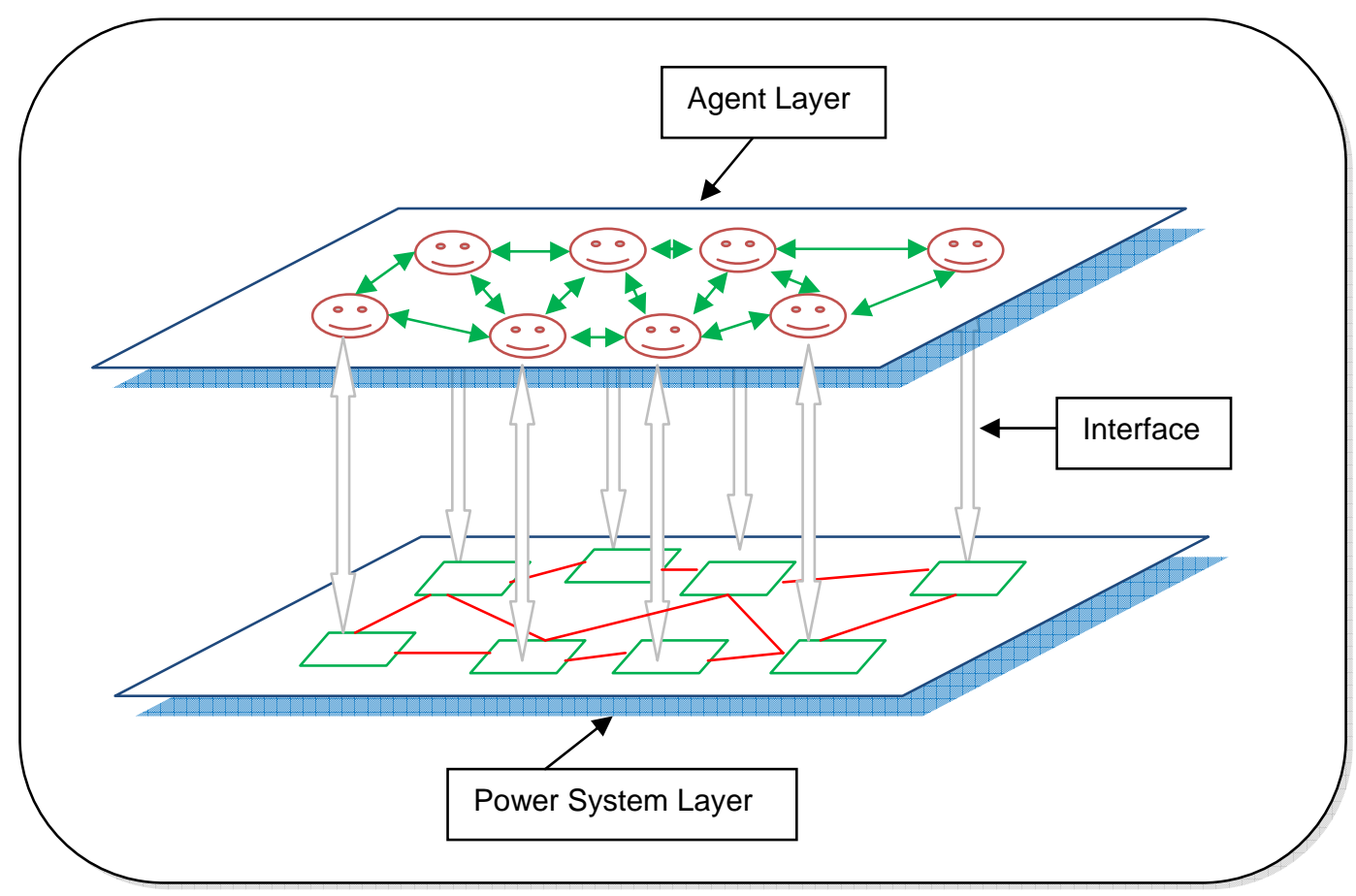

Figure 3-1: Architecture of the Simulation Model.

\subsection{MATLAB ${ }^{\circledR}$}

MATLAB ${ }^{\circledR}$, Matrix Laboratory, is a high level technical language and numerical computing environment for algorithm development, data visualization, data analysis, and numeric computation. MATLAB solves technical oriented tasks faster than traditional languages such as $\mathrm{C}, \mathrm{C}++$, and FORTRAN [49]. MATLAB was first adopted by control design engineers, later it is been used in many other scientific applications including image processing, communications, etc. Some of the key features of MATLAB are listed as followed [63],

- Developing environment for managing code, files, and data

- High level language for technical computing.

- Tools for creating custom graphical user interfaces.

- Functions for integrating MATLAB based methods to external applications, such as C, C++, FORTRAN, Java, and Microsoft Excel.

- 2-D and 3-D graphics functions for visualizing data. 
MATLAB is more suitable for engineering applications because of its operations based on matrix and vectors. MATLAB is faster in engineering tasks, because of the basic tasks like variable declaration, memory allocation, and data type specifications are default. MATLAB uses Object Oriented Programming (OOP) thus, adds the object oriented features to it. This feature enables to develop complex technical computing applications easily. When using well defined classes, object-oriented programming can significantly increase the code reuse and, make program easier to maintain and extend. Besides the OOP attributes, MATLAB has Graphical User Interface (GUI) tool to create and visualize customized interfaces.

In this thesis work MATLAB object-oriented programming is used to create the reconfiguration algorithms and the plotting functions. In addition to that, a graphical user interface for the reconfiguration has been developed using MTALAB GUI.

\subsection{MATPOWER}

MATPOWER is a MATLAB based power system simulation package. It is a collection of MATLAB m-files to solve the load flow and optimal power flow problems of power system [64]. It is indeed a simulation package for researchers and educators, where one can easily make modifications to the original code according to one's application. Ray D. Zimmerman is the main contributor for this power simulation software. The need for MATLAB based power flow and optimal power flow born out of the computational requirements of the PowerWeb project at Cornell University [65]. MATPOWER is an open source software and freely downloadable at [64]. MATPOWER is used for load flow calculations of Circuit of the Future (CoF) distribution network in this thesis work.

\subsection{Java Agent Development Framework}

JADE (Java Agent Development Framework) is a software package for developing agentbased applications in compliance with Foundation for Intelligent Physical Agents (FIPA) specifications for interoperable and intelligent multi-agent systems [66]. JADE is a middleware application completely developed using Java language. JADE has extensive 
graphical tools for the debugging and deployment phases. The key advantage offered by JADE is its Graphical User Interface (GUI), through which one can visualize the development phases and agent communications.

Today, JADE is the most popular agent middle-ware that implements an agent platform and a development framework. JADE was developed in 1998 by the Research and Development department of Telecom Italia, in 2000. JADE is released as open source software under the license of Lesser General Public License (LGPL). JADE software is freely downloadable from [66] with source code, documentation, and some wealthy information.

\subsubsection{JADE Architecture}

JADE architecture has several agent containers that can be distributed over the network. Agents live in the containers are the Java processes which provide the JADE run-time and, all the services needed for instantiating and executing the agents [67]. In every JADE framework, there is a special container, called the main container. It is the first container to be launched and all other possible containers must join to a main container by registering with it. By default main container is named 'Main Container' and the remaining containers are named 'Container-1', 'Container-2', etc. The main duties of main container are listed below [67],

- Creating and Managing the container table (CT), which is the registry of the object references and transport addresses.

- Creating and Managing the Global Agent Descriptor Table (GADT), which is the registry of all agents present in the JADE platform including their current status and location.

- Hosting the AMS agent, which provides agent management and white page service.

- Hosting the DF agent, this provides default yellow page service. 


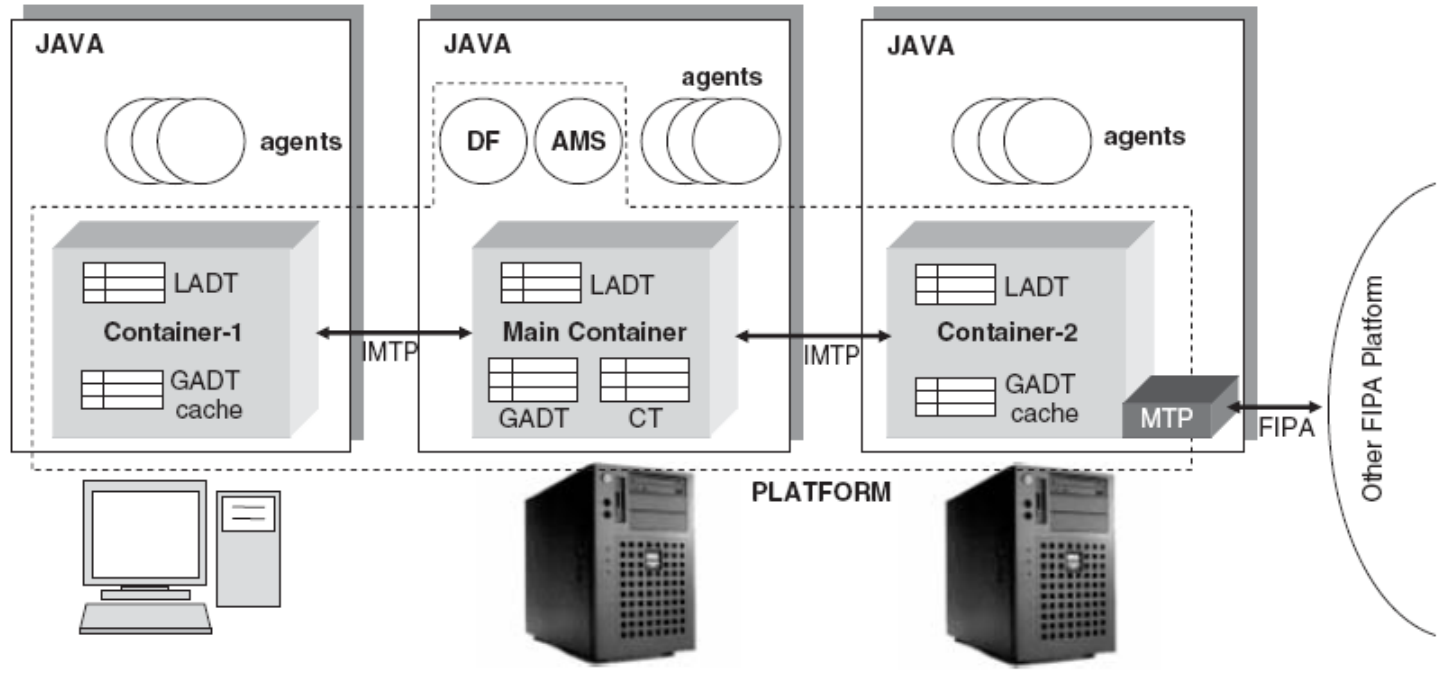

Figure 3-2: Relationship between main architectural elements [67].

The two main agents named Agent Management System (AMS) and Directory Facilitator (DF) are instantiated when the main container is launched.

- $\quad$ The Agent Management System (AMS)

Agent Management System is the special agent which supervises and monitors the whole platform. It is the main hub for all agents present in the container in order to access the white pages of the platform and to get Agent Identifier (AID). All agents life cycle are managed by AMS. The registering process of all agents with AMS is done automatically by JADE at start-up.

- The Directory Facilitator (DF)

Directory Facilitator (DF) agent implements the yellow age service. These services are used by any agent wishing register its services or search for other available services. Multiple DFs can start concurrently in order to distribute the yellow page services across several domains. 
Besides AMS and DF agents, the one more important service according to FIPA is a Message Transport Service (MTS). An MTS manages all message exchanges within and between platforms. It supports several Message Transport Protocols (MTPs) to promote interoperability between different non-JADE platforms [67]. There are several other agent tools such as, Dummy Agent, Sniffer Agent, and Introspector Agent to facilitate the developing and debugging of agent platforms.

- The Dummy Agent

The dummy agent is a very simple agent which is used to simulate other agent's behavior by sending the custom Agent Communication Language ( $A C L$ ) messages. When an application agent has been launched, a Dummy agent can be used to stimulate it by sending user-specified messages and analyzing its reaction in terms of received messages.

- The Sniffer Agent

This is a very useful agent tool in terms of debugging the project. This agent tool enables to visualize conversations between agents. The conversations between agents are shown by arrows; each arrow contains the message content. In the Sniffer agent GUI, different agent communications can be marked with different colors.

- The Introspector Agent

The Introspector agent is useful to debug the behavior of a single agent. This agent will control and monitors the agent sent and received messages, scheduled behaviors of agent, and the agent life cycle. In simple words, this agent is responsible for introspection of agent execution.

The following figure shows a typical JADE GUI for the remote management, monitoring and controlling of the agents present in platform. 


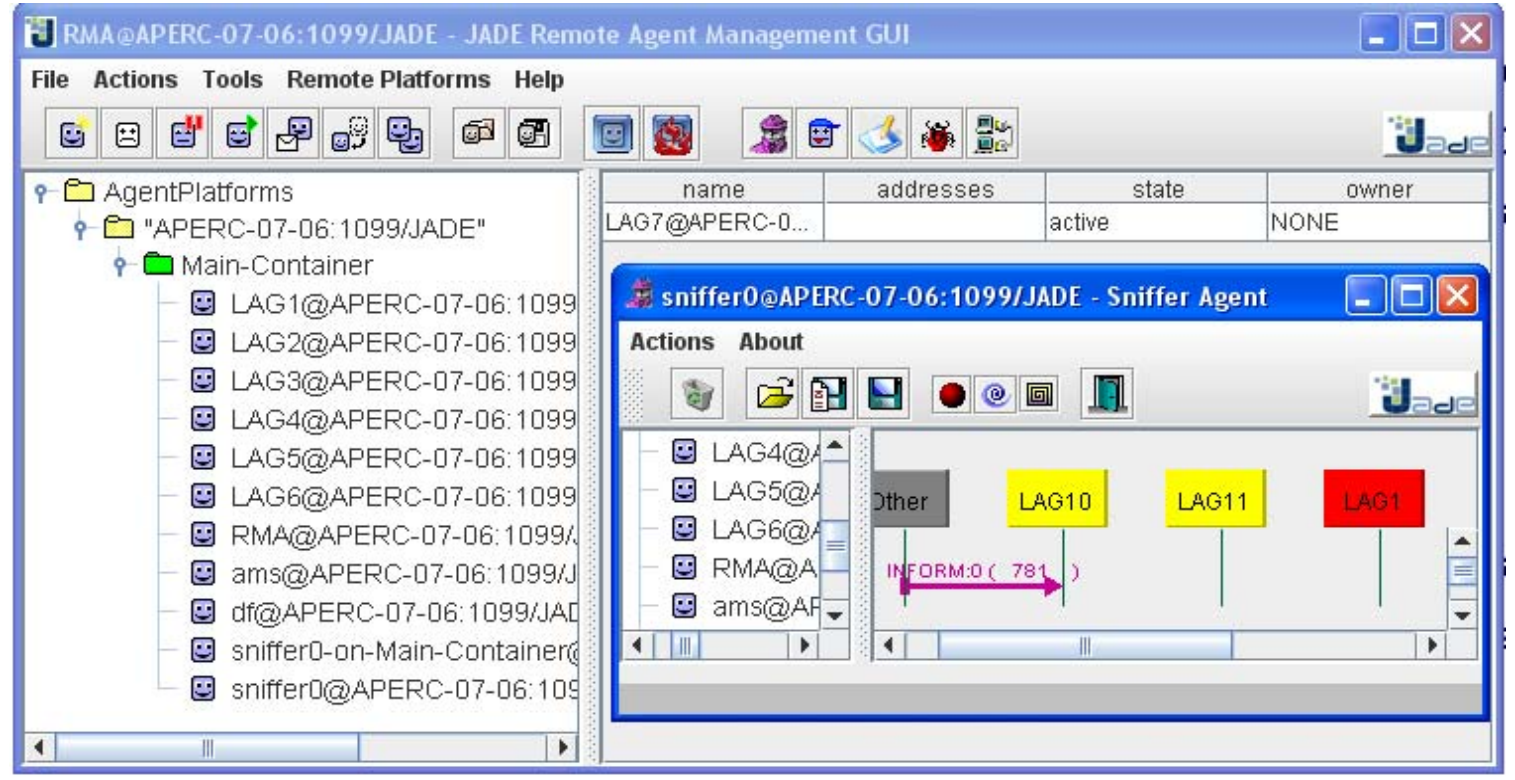

Figure 3-3: JADE Graphical User Interface (GUI).

The following figure shows the output of JADE run-time environment, when JADE is launched.

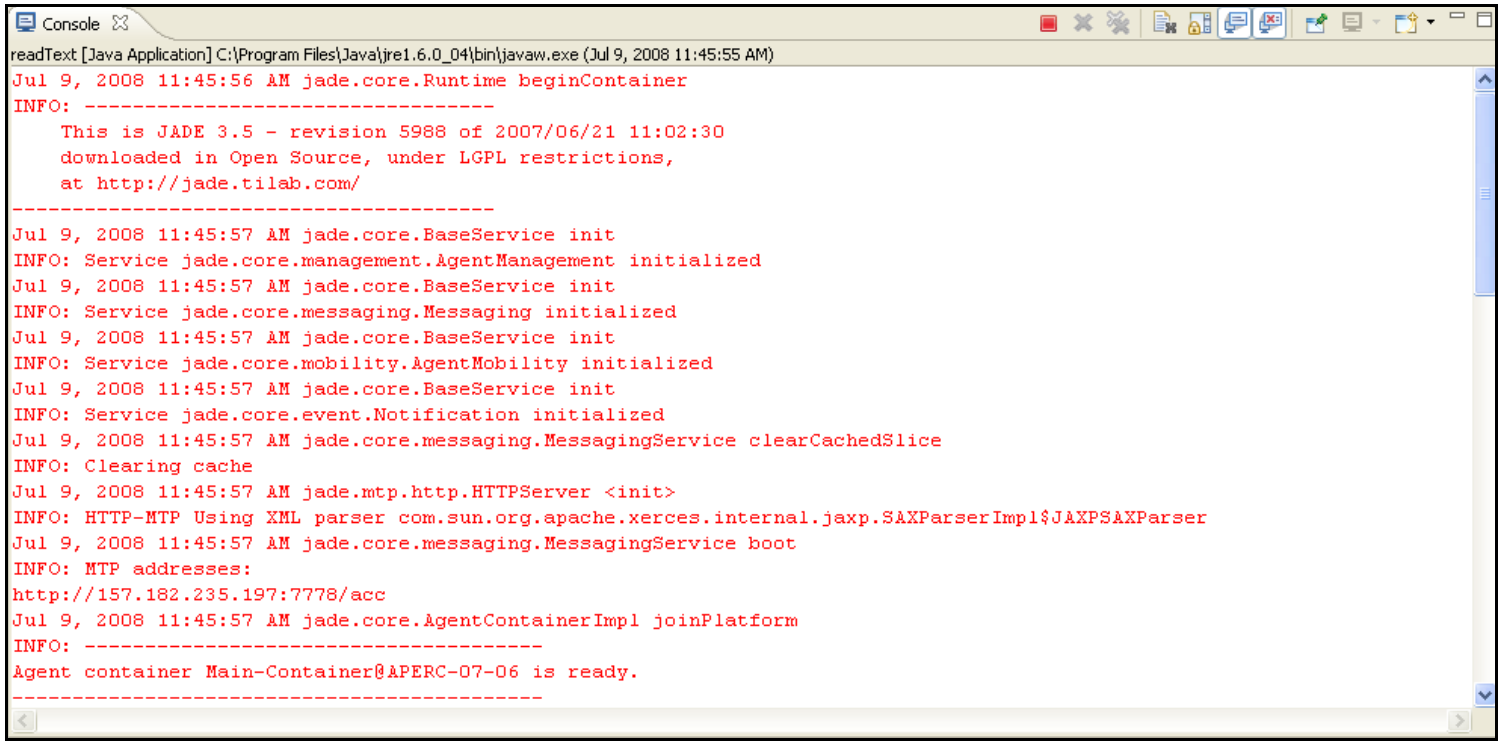

Figure 3-4: JADE run-time environment. 


\subsubsection{JADE Programming}

JADE programming is similar to the standard JAVA programming. The creation of an agent is done by a simple class jade.core.Agent and implementing the setup() method. The setup() method intended to include agent initializations. The actual job performed by the agent is defined within the 'behaviors' of agent. The 'behavior' property tells the agent about its typical operations. The typical operations, an agent performs in its setup() method are: showing a GUI, opening the connection to database, registering the services to provide the yellow pages, and starting the initial behavior [67].

According to FIPA specifications each agent is identified by a unique 'agent identifier' in Java Agent Development Framework. In JADE, the getAID() method is used to retrieve the local agent identifier. The AID object includes a globally unique name (GUID) and a number of addresses. These AIDs are very important entities in agent communication.

As discussed earlier agent communication is the most important feature of Multi-agent systems. Each agent that is created has a mailbox where the messages are passed in a queue when it receives messages from other agents. The messages are compliant with FIPA standards. The ACL messages are sent to one agent from another agent by using send() method. The receiving agents can receive the message by calling receive() method. Agent will be alive even after completing all its responsibilities. doDelete() method is used in order to terminate it.

JADE has been used in this thesis work for developing the agent platform for the Circuit of the Future (CoF) distribution system.

\subsection{Distributed Engineering Workstation@ (DEW)}

Distributed Engineering Workstation is a distribution system software and an open architecture environment with integrated data and applications, analysis, design, and operation modules access database data and exchange data through common functions. The distribution engineering tool is designed to meet the analysis, planning, design, and operation needs of today's distribution systems. DEW offers myriad tools such as, power 
flow, load estimation, line impedance calculations, fault analysis, capacitor placement, phase balancing and many more [68]. Some of the add-ons of this software can be bought separately from the vendor. The open architecture allows external application modules to be added to the workstation.

Executive, Database, Graphical User Interface (GUI), Application Programmer Interface (API), and Application modules are the major components of the Distributed Engineering Workstation (DEW) [69]. These major functional parts of the DEW are depicted in the following figure.

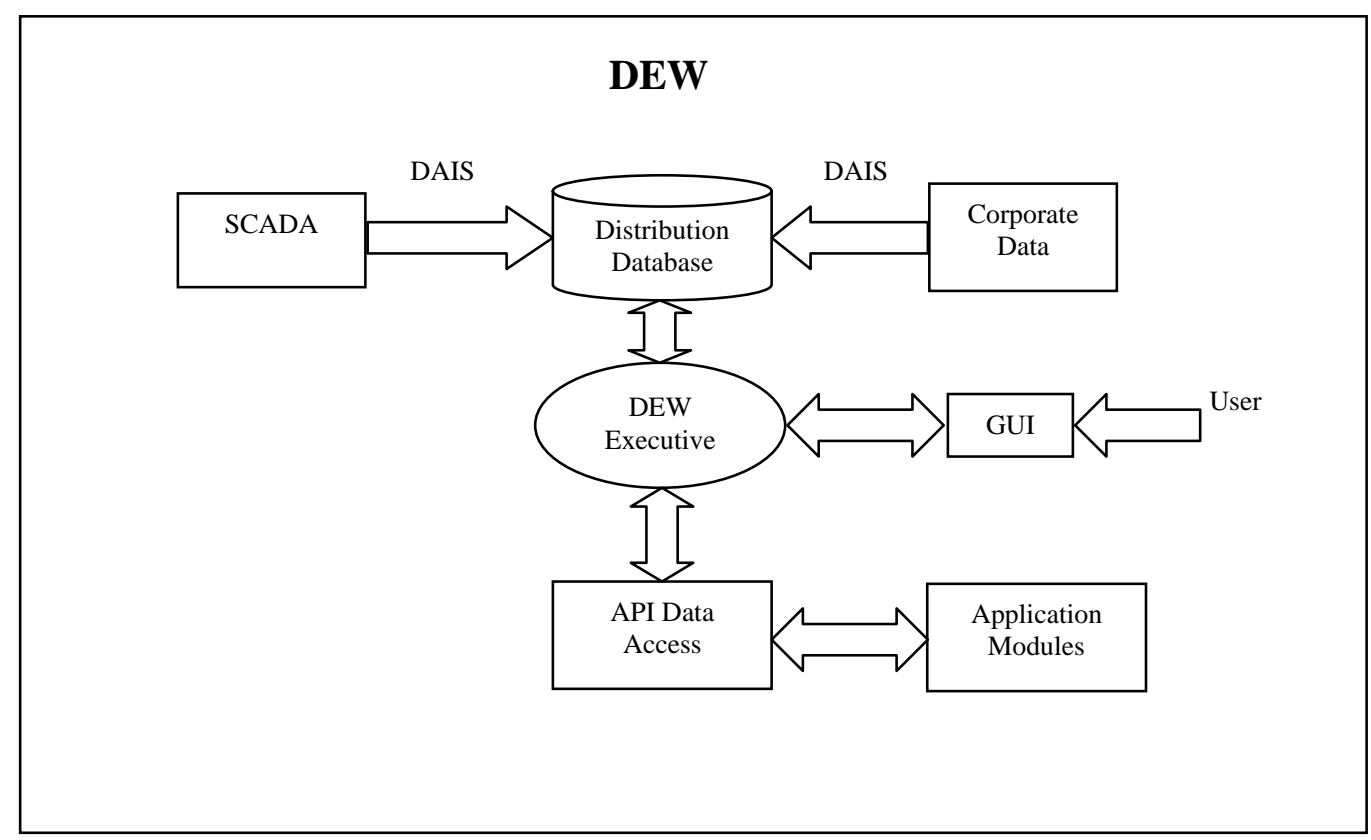

Figure 3-5: Organizational structure of DEW [69].

The main operations of the workstation are managed by Executive. It provides interface between API, GUI, and database modules. The key feature of API is to integrate the application modules into the framework without disturbing other parts of the workstation. All the applications utilize the GUI functions for user interaction and display. The standard data schema available in DEW makes data accessible and shareable by all applications. The data from Supervisory Control and Data Acquisition System (SCADA) such as, financial, statistical, geographical, operations data are utilized for the application through an interface called, Database Access Integration Service (DAIS). 
The following figure shows the Graphical User Interface (GUI) of DEW. The right side pane of the DEW main page has different power system entities. These entities can be dragged into the DEW workspace to build the one-line model of the distribution system. In this thesis work DEW has been used to model the Circuit of the Future (CoF) distribution network.

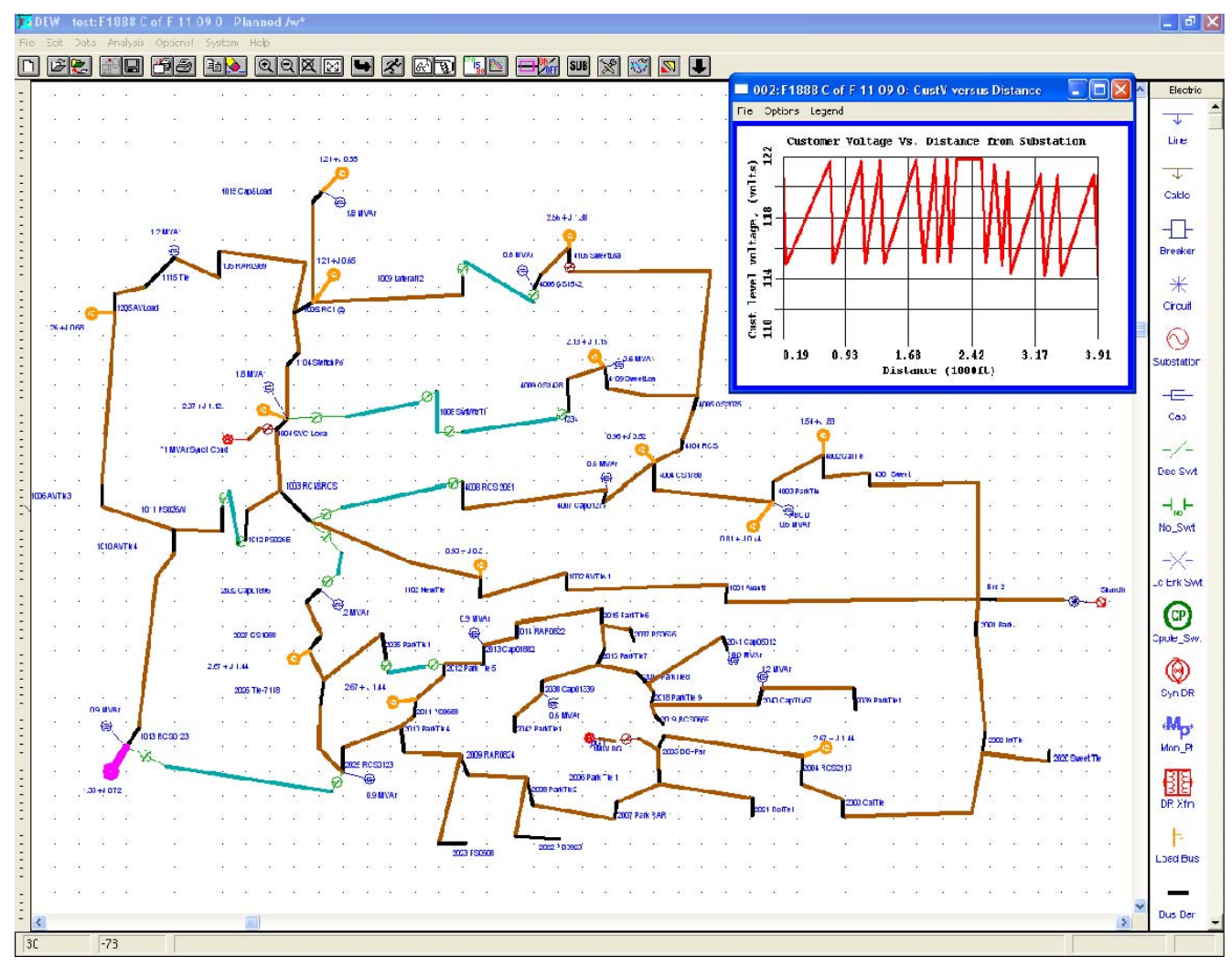

Figure 3-6: Distributed Engineering Workstation's Working Environment.

\subsection{Power World Simulator}

Power World simulator is an interactive power system simulation package designed to simulate high voltage power system operation. The software contains a highly effective power flow analysis package capable of efficiently solving systems with up to 100,000 buses [70]. Some of the key tools offered by Power World Simulator are listed below, 
- Load Flow Analysis

- Optimal Power Flow (Linear programming method)

- Security Constrained Optimal Power Flow (SCOPF)

- Available Transfer Capabilities (ATC)

- PVQV curve tool

- Transmission line Parameter Calculator

- Contingency Analysis

- Fault Analysis

This software is a very good tool for modeling the power system. The key feature of this software is its graphical user interface (GUI), where one needs to drag and drop the power system elements to build up one's own case. Different tools available in Power World Simulator are listed above. The following figure shows the snapshot of one line diagram of Circuit of the Future (CoF) created in Power World Simulator.

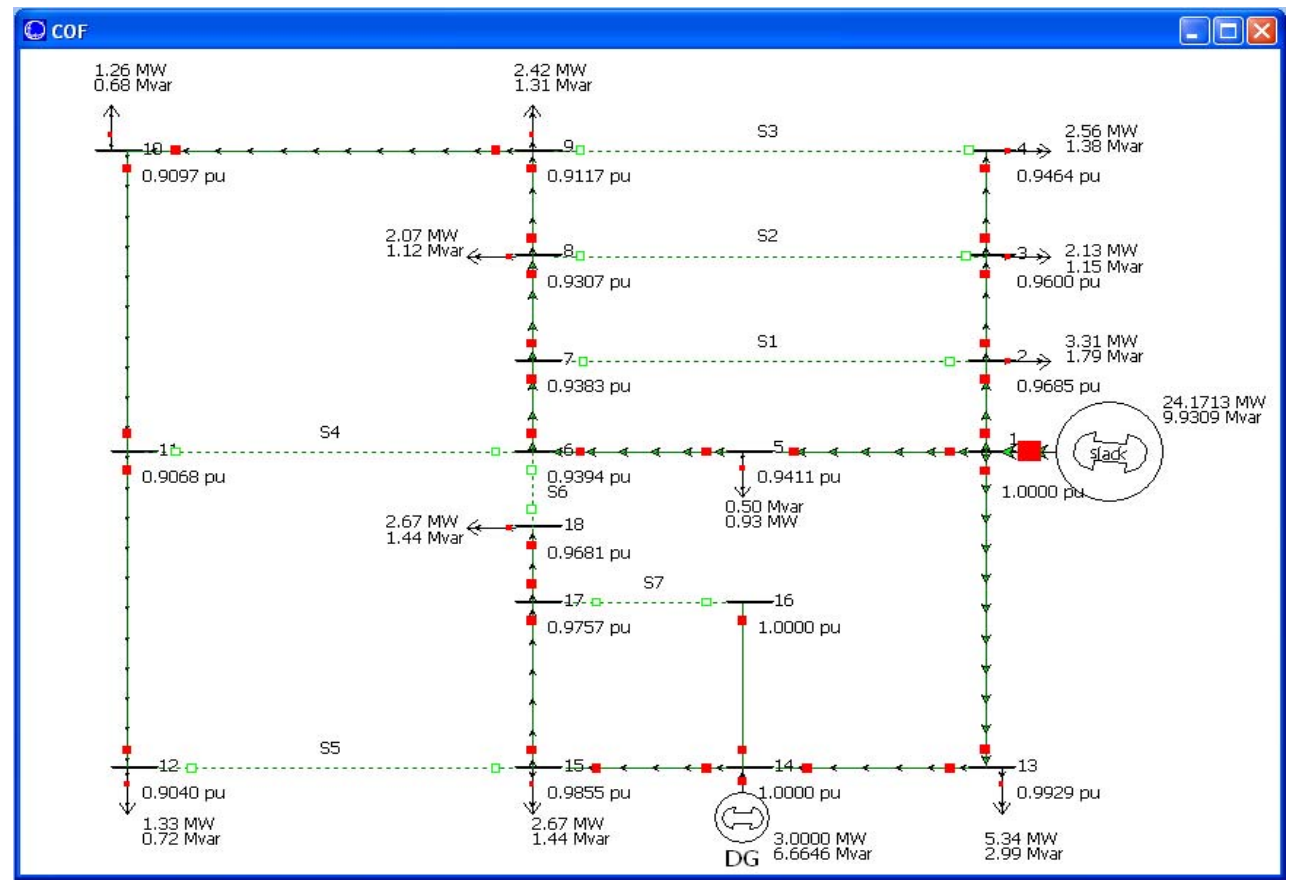

Figure 3-7: Snapshot of Circuit of the Future one line diagram 
Power World Simulator has a very good facility of visualizing the load flow results. Every time we run the load flow all the results will be stored and viewed through a GUI entity called Model Explorer. The model explorer has a explore pane, which contains a list of the objects contained in the power system model. The entries in the explore pain which are grayed out indicates that the elements are not defined in the model. The explore pain again divided into eight sub folders named, Recent, Network, Aggregation, Solution Details, Case Information and Auxiliary, Contingency Analysis, Optimal Power Flow, and Transient Stability. The following figure shows a snapshot of Power World Simulator's Model Explorer.

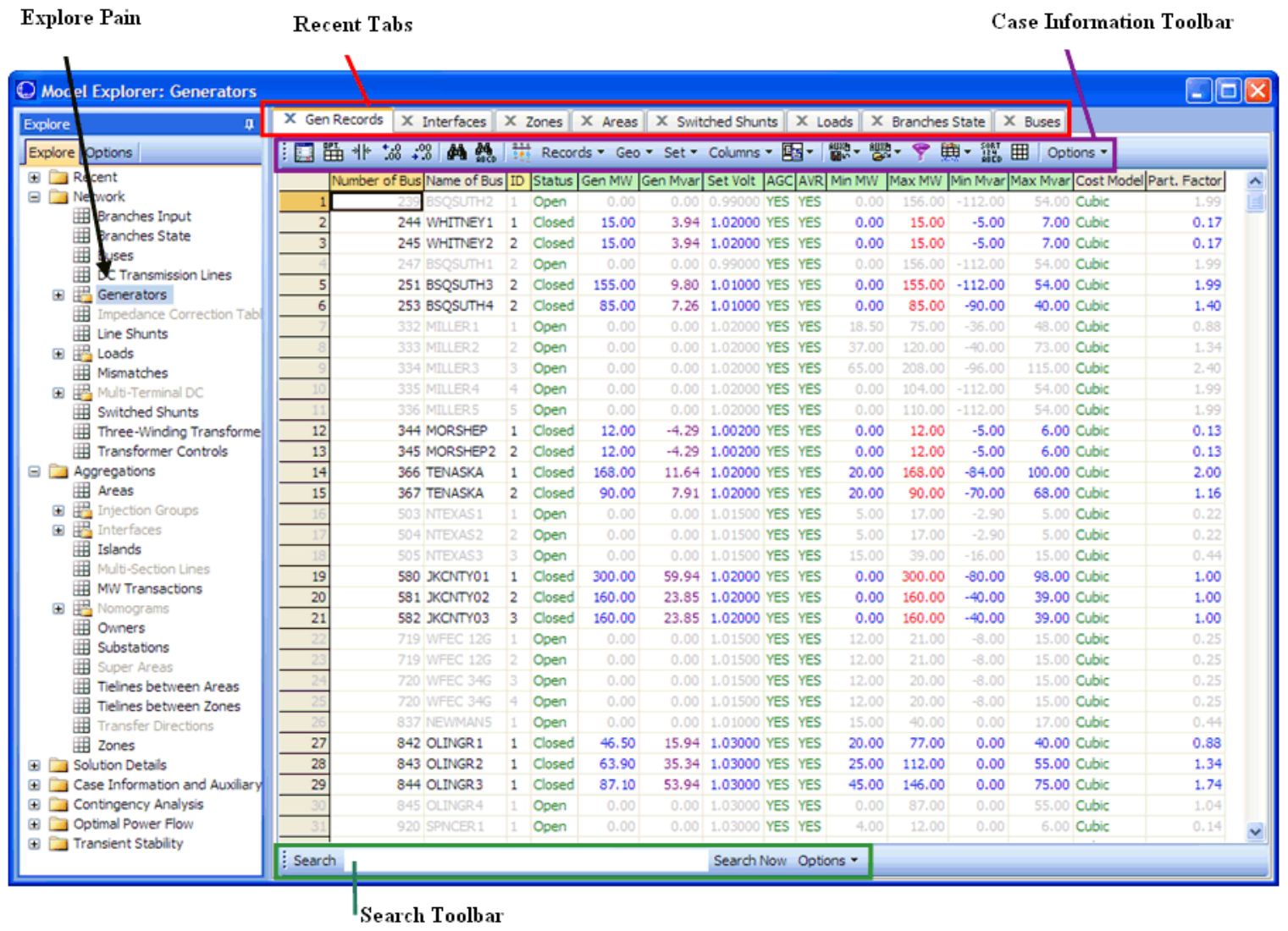

Figure 3-8: Snapshot of Model Explorer of Power World Simulator [70]. 


\section{Chapter 4}

\section{Mathematical Model AND}

\section{Algorithms for MAS}

This chapter mainly explains the mathematical model used behind the Multi-agent framework in this thesis. Multi-agent architecture has its own mathematical model to serve as its foot-print. Once the mathematical model is build then it can be translated into software packages for simulation purpose. This chapter presents a set of new algorithms for fault detection and reconfiguration. The fault detection algorithm is based on Graph theory, and the reconfiguration algorithm is based on load-flows.

\subsection{Mathematical Model}

\subsubsection{Graph Theory}

Graph theory is the study of graphs used extensively in the fields of mathematics and computer science. A 'graph' is defined as, collection of vertices or 'nodes' and collection of edges that connects pairs of vertices [71]. The graphs are primarily categorized as either directed graphs or undirected graphs. The major difference between above two graphs is, the direction between two vertices. Graph theory has several applications such as network 
analysis, study of molecules in physics and chemistry, and social network analysis in sociology. The main application of graph theory lies in modeling and analyzing traffic networks. Power system is a vast network consisting tens of thousands of vertices, can be modeled using graph theory.

\subsubsection{Graph Theory representation of Power System}

The power system network is modeled as a graph $G$ as shown in the Figure $4-1$. It has a single parent node. The power distribution feeders form the spanning tree $T$. Each node in graph $G$ represents power system elements such as loads, sources and buses. These nodes are connected by edges. The edges in spanning tree $T$ represent transmission lines connecting every pair of buses. The edges in $G$, but not in $T$ represent switches. These switches are used for restoration purposes during the fault and left open during normal healthy conditions of power grid. In the proposed MAS, agents are place at each and every node and switch of the power distribution network. The following table describes some of the key notations used in the mathematical model.

\begin{tabular}{|c|l|}
\hline Notation & \\
\hline$G$ & Graph, represents the power network. \\
\hline$T$ & Spanning tree, represents radial feeders. \\
\hline$N$ & Set of nodes in the graph. \\
\hline$E$ & Set of directed edges in the graph, represent power lines. \\
\hline$S$ & Power source, represents substation or a distributed generator. \\
\hline$S(G)$ & Set of edges in $G$, but not in $T$, represents set of switches in the system \\
\hline
\end{tabular}

Table 4-1: Description of the notations used in Mathematical model. 


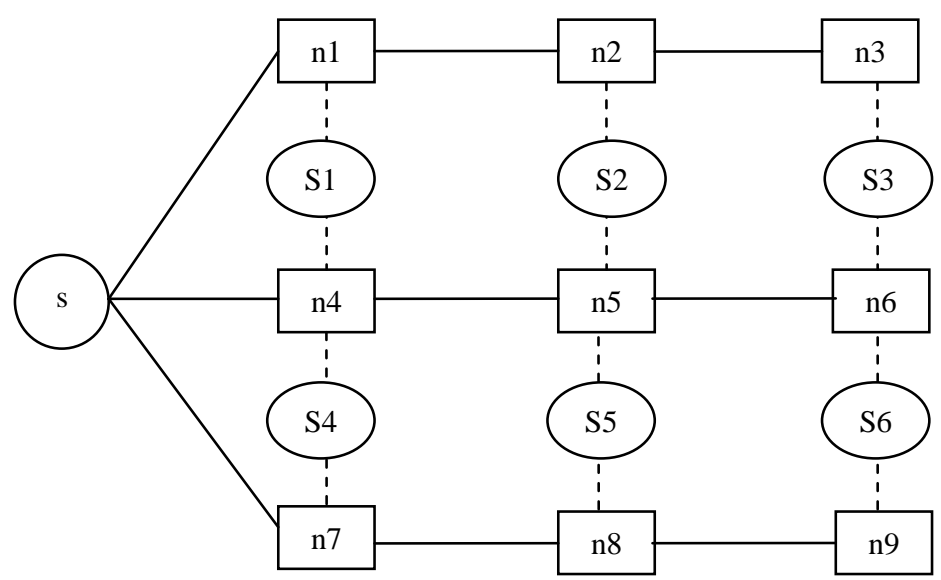

Figure 4-1: Graph model of power distribution system.

In the above graph model $s$ represents the substation. Each branch $s \rightarrow n_{1} \rightarrow n_{2} \rightarrow n_{3}$, $s \rightarrow n_{4} \rightarrow n_{5} \rightarrow n_{6} \quad, s \rightarrow n_{7} \rightarrow n_{8} \rightarrow n_{9}$ forms the spanning tree. The edges in the graph, but not in the trees are switches, S1-S6. In the spanning tree every node except the root node has in-degree ${ }^{1} 1$. That is, each node $n$ in T can be reached by a directed path in T form the source $s$ to $n$.

For each edge $e \in E(T)$, let

$$
S(e)=\{X: X \subseteq S \text {, such that }(T-e) \bigcup X \text { is a connected graph }\}
$$

\footnotetext{
${ }^{1}$ In-degree of a vertex in a graph is defined as the number of edges coming into the vertex.
} 


\subsection{Fault Detection Algorithm}

The following section describes about the Fault Detection algorithm used in this thesis work. Fault detection is the first thing in restoring the faulty power network. Major input to this algorithm is power flowing into each load.

Let us assume that the fault location detected by the node agents is at $n_{f}$. Suppose a node agent $n$ identifies that the power flowing into its node is zero, then on the unique directed path from $s$ to $n$, say $s \rightarrow n_{1} \rightarrow n_{2} \rightarrow n_{3} \rightarrow \ldots \ldots \rightarrow n_{k}$, where $n=n_{k}, \quad n_{k}$ will request $n_{k-1}$, whether it has power. Subsequently, $n_{k-1}$ will request $n_{k-2}$ for its power. When for some $i, n_{i}$ does not have power, but $n_{i-1}$ has power, the fault edge can be identified as, $e_{f}=\left(n_{i-1}, n_{i}\right)[2]$.

Once the fault location is identified, the edge $e_{f}$ is isolated and agents that are controlling each switch $X \in S\left(e_{f}\right)$ will communicate and coordinate with each other to decide on the particular switch or switches that have to be switched on, so that $\left(T-e_{f}\right) \cup X$ will continue to be a fully supplied network [2].

\subsection{Fault Reconfiguration \& Restoration Algorithm}

The reconfiguration algorithm employed in this thesis work is completely based on loadflows. This algorithm is embedded inside the Global agent for reconfiguration of the faulty network. Depending on the number of faults in the system, there are three different algorithms to reconfigure the power distribution network. They are,

- Reconfiguration algorithm for single fault.

- Reconfiguration algorithm for double fault.

- Reconfiguration algorithm for three faults. 


\subsubsection{Reconfiguration algorithm for single fault}

The fault location, $e_{f}$, has been found from the previous algorithm and possible switches are explored through this algorithm for reconfiguration. This algorithm searches for all the possible combinations of switches, which has to operate in order to reconfigure the circuit. If there is voltage violation in the circuit, the algorithm employs shunt compensation and load shedding methods to improve the voltage profile. When there are multiple solutions available for reconfiguration, the best solution is chosen in regards of voltage profile and less power losses. Each time the algorithm executes a scenario, the solution is stored in a database. Next time when the same scenario happens the reconfiguration would run from the database rather than running through the algorithm. The algorithm is described through the flowchart in the Figure 4-2.

\subsubsection{Reconfiguration algorithm for two faults}

The solution from single fault reconfiguration algorithm is used in this algorithm. When we use solution form above algorithm, some times the load flow will not converge. In this case this algorithm itself searches for additional switches to reconfigure the faulty circuit. If the reconfigured circuit has voltage violations, then the algorithm employs shunt compensation and priority based load shedding for voltage profile improvement. Similar to above algorithm this algorithm also has a database to store the solutions of the scenarios. When there are multiple solutions, the best solution in regards to voltage profile and less power losses is chosen.

\subsubsection{Reconfiguration algorithm for three faults}

This algorithm is also similar to the above two fault reconfiguration algorithm. The solution from the single fault reconfiguration algorithm is used in this algorithm. The voltage control is done in the same manner using shunt compensation and priority load shedding. 


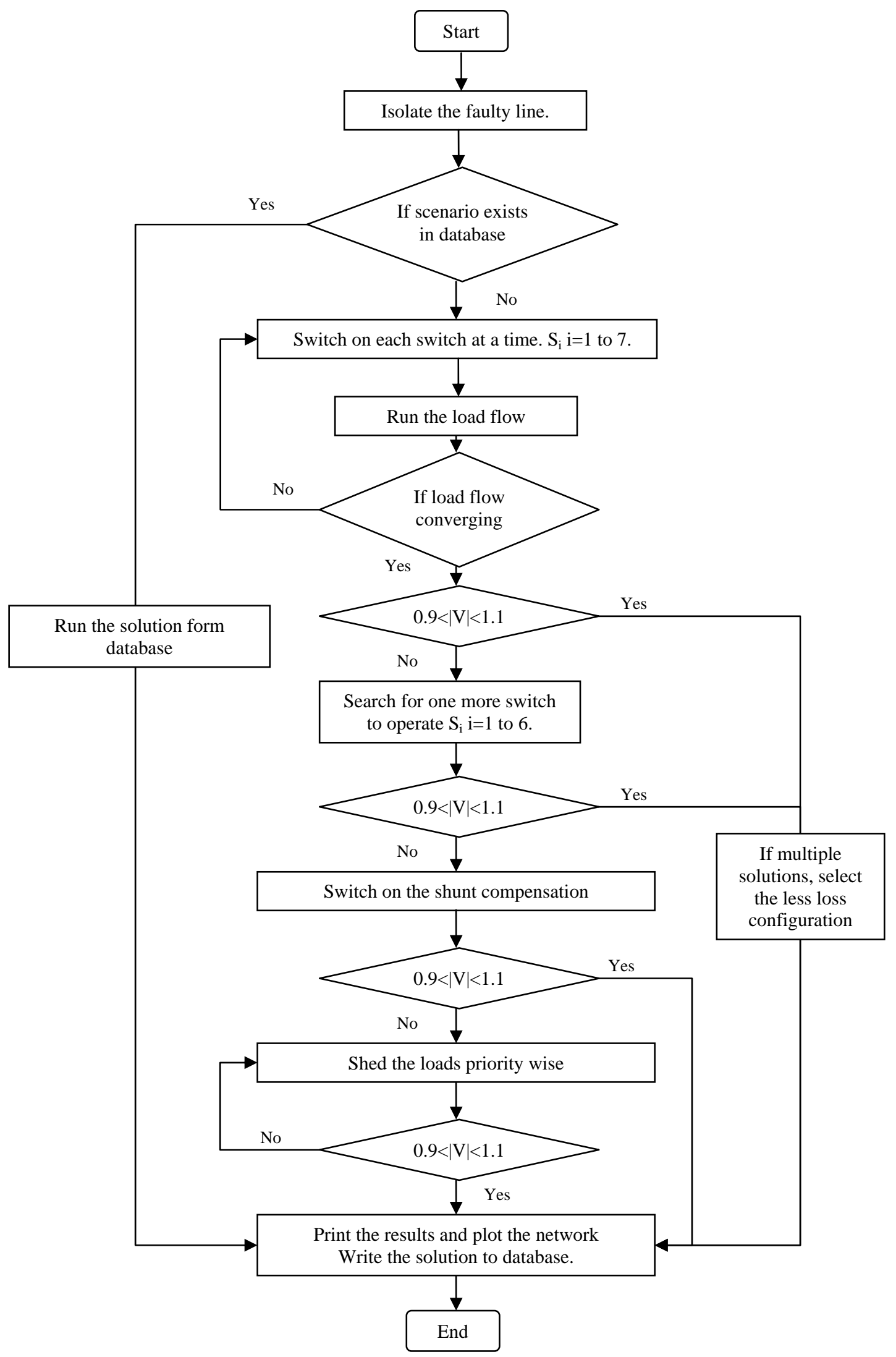

Figure 4-2: Flow Chart for Fault Reconfiguration 


\subsubsection{Objective functions and Constraints}

The main objective functions of the reconfiguration algorithm are

- Isolating the faulty part of the network and restoring the remaining part of the network with good voltage profile.

- Always supplying the critical loads.

These objective functions are subjected to following constraints.

- Power flow across the transmission line should be kept under its thermal limits.

- Voltage magnitudes of all buses should be kept under the limits of $0.9<|\mathrm{V}|<1.1$.

- Less loss configuration is chosen for the reconfiguration when multiple solutions exist.

The reconfiguration topology achieved will comply with the objective functions and the constraints listed above. There is a major consideration for voltage profile of the reconfigured network. The voltage control in the algorithm is achieved by means of shunt compensation. When the shunt compensation is not sufficient to meet the voltage limits, it starts shedding the non priority loads. Algorithm employs a database technique to store all the scenarios such that, when the same scenario happens the reconfiguration runs form database rather than through algorithm. This technique greatly reduces the algorithm execution time, which is the major concern in algorithm efficiency. 


\subsection{Illustration}

In this section a particular scenario is illustrated for better understanding of the reconfiguration algorithm. This illustration is based on simplified CoF circuit as shown in Figure 4-3. In this figure, $N_{i}$ are the nodes, $L_{i}$ are the loads, $S_{i}$ are the switches, $\mathrm{s}$ is the substation and DG is the Distributed Generator modeled as a backup source. Let us assume that the fault is in $e_{f}=\left(N_{1} N_{2}\right)$.

The load agents in $L_{1}, L_{2}$ and $L_{3}$ will detect that there is no power flowing into their respective loads. Hence, these three load agents will communicate with their respective neighbors in the incoming tree from $N_{4} \rightarrow N_{3} \rightarrow N_{2} \rightarrow N_{1}$ for their flows. When all these nodes detect that they don't have power flow, the MAS can locate the fault in $N_{1} N_{2}$. The load agents will report this fault location to the global agent, which has the potential to reconfigure the circuit. This agent has the reconfiguration algorithm discussed in section 4.3. The algorithm execution is described in following steps.

- Isolates the faulty link, i.e. link 1-2.

- There are three switches which can individually reconfigure the network. They are, $S_{1}, S_{2}$ and $S_{3}$.

- By switching on the above switches individually, the voltage limits are not met.

- Now the algorithm searches for one more switch to operate by keeping the $S_{1}$ on, which can reconfigure the network with no voltage violation. Same procedure repeats with $S_{2}$ and $S_{3}$.

- Finally, algorithm finds two solutions which have no voltage violations. They are $\left\{S_{2}, S_{5}\right\}$ and $\left\{S_{1}, S_{5}\right\}$.

- Out of this two solutions, $\left\{S_{1}, S_{5}\right\}$ is chosen because of the less losses.

- Finally the results are printed and the reconfigured network is plotted. 


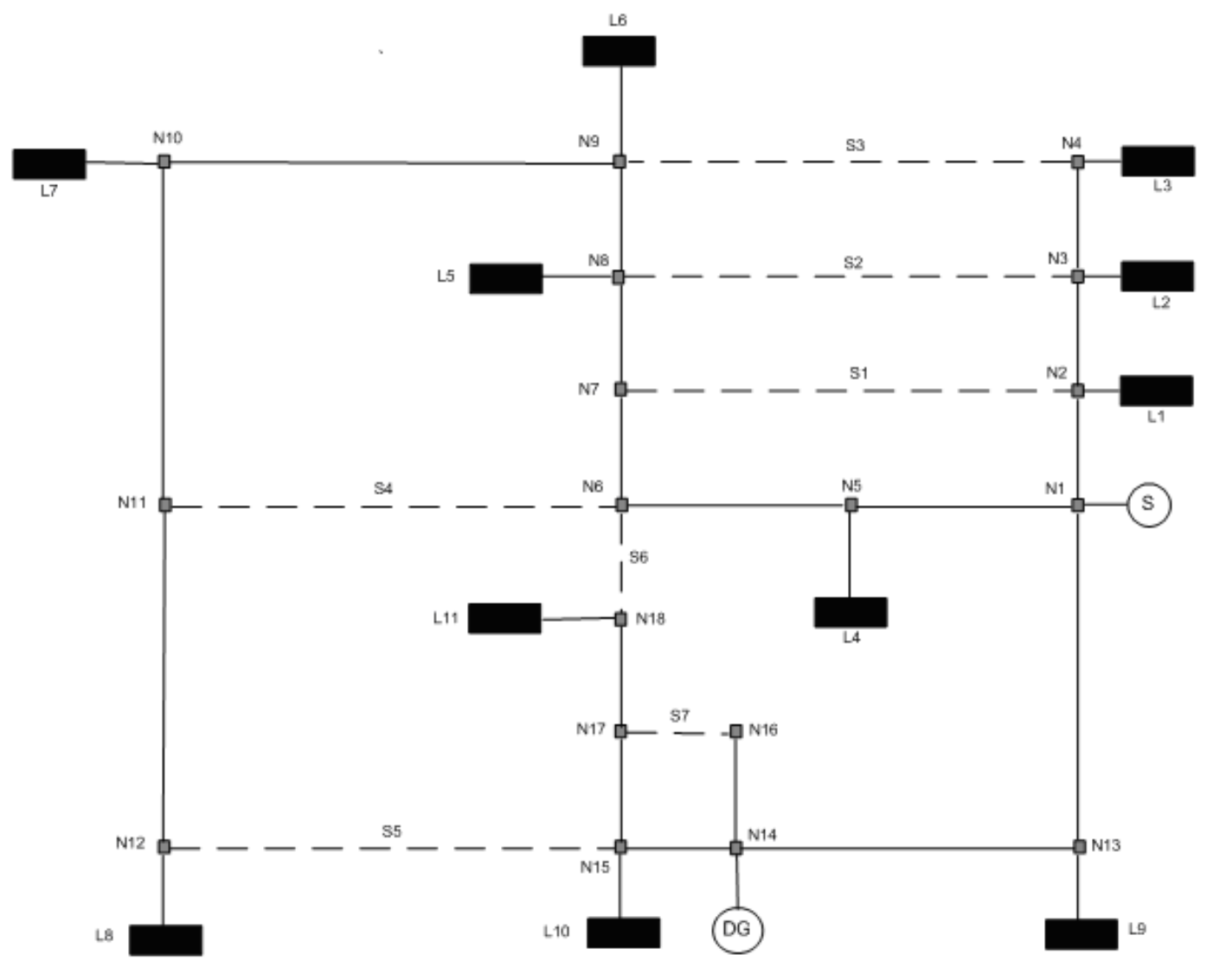

Figure 4-3: Representation of Simplified CoF.

As discussed above, the algorithm comes up with the best possible switch combination $\left\{S_{1}, S_{5}\right\}$ for the reconfiguration, when there is a fault at link 1-2. The same steps are explained elaborately in the section 5.4 .1 in the next chapter. 


\section{Chapter 5}

\section{Simulation AND Results}

In this chapter, the fault detection algorithm and the reconfiguration algorithm discussed in the previous chapter are tested on simplified CoF distribution system. The fault detection algorithm is developed in JADE, where as the reconfiguration algorithm is developed in MATLAB. Power World Simulator and DEW have been used for modeling and designing the simplified CoF.

\subsection{Proposed MAS Architecture}

The proposed MAS architecture for fault detection and reconfiguration is illustrated in Figure 5-1. According to the hierarchy, there are mainly two categories of agents available in the proposed MAS architecture. They are,

- Local Agents

- Global Agent.

Load Agents (LAGs) and Switch Agents (SAGs) fall into the category of local agents. These agents represent loads and switches in the power distribution system respectively. Any load agent can be associated with a number of switch agents. Whenever there is a fault in the system, load agents and switch agents start communicating and coordinating each other in order to locate the fault location in the network. Once the fault is detected, the fault location is reported to the Global Agent (GAG) by load agents. The 
reconfiguration algorithm is embedded inside the GAG, such that whenever it receives fault location it starts reconfiguring the network to reroute the power to critical loads.

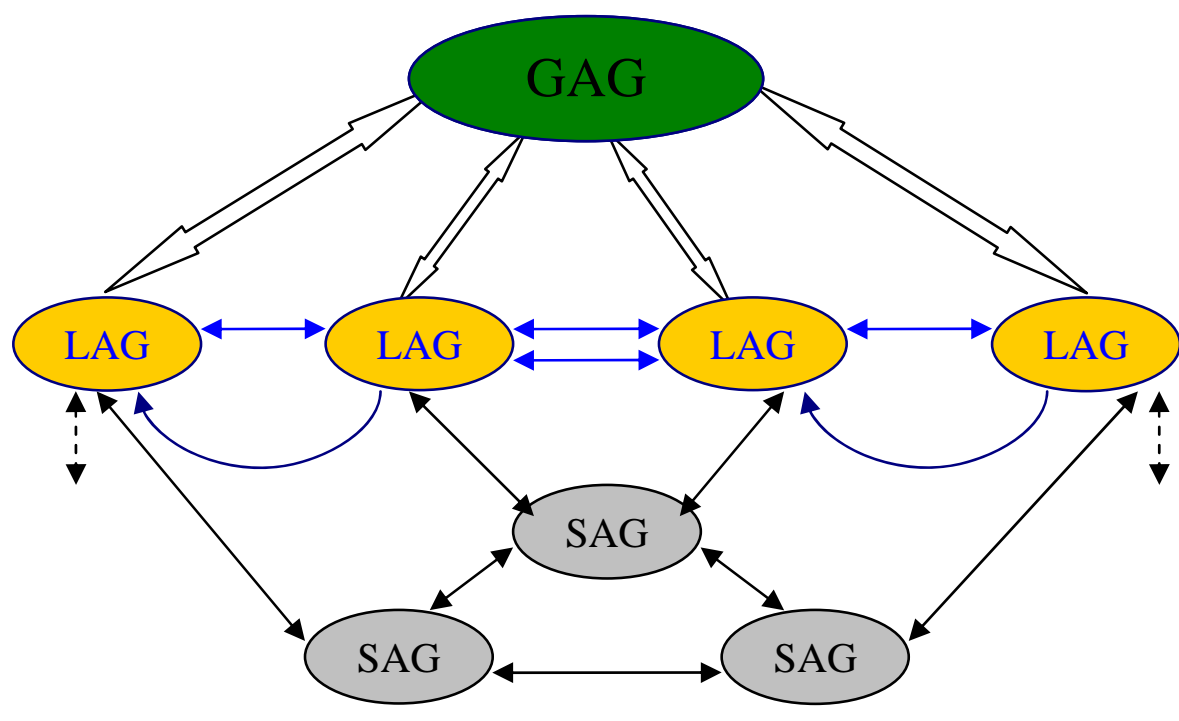

\section{LAG - Load Agent; SAG - Switch Agent; GAG - Global Agent} $\leftrightarrow$ Communication

Figure 5-1: Proposed Multi-agent system architecture.

The local agents such as LAGs and SAGs only can communicate to its neighboring agents. These agents will have local information pertaining to that particular region only. Local agents communicate and coordinate in the sense of decentralized manner, where as the Global agent operates much in centralized manner. Because of this fact, the proposed MAS architecture is a hybrid MAS architecture. In this hybrid architecture the fault detection is done in a decentralized manner and the fault reconfiguration is done in a centralized fashion. The main responsibilities of each agent can be summarized as below,

- Load Agents (LAGs)

These agents will continuously monitor the loads present in the system. Whenever they sense no power, they start communicating their power flow information to their neighboring agents. Finally they will come up with the fault location based on fault detection algorithm. Once the fault is detected, the fault location is reported to Global agent (GAG). 
- Switch Agents (SAGs)

These agents are the dummy agents, which keep status of the switch position and coordinate with the GAG and LAGs during fault detection and reconfiguration.

- Global Agent (GAG)

The Global agent is very important entity in fault reconfiguration. It has all the information regarding network and has the capability of doing load flows. The reconfiguration algorithm is embedded inside the GAG. GAG will call its Voltage Control routine, whenever there is a need for voltage control. Every time GAG runs the scenario it stores it in its database for learning. When the same scenario happens in the future, GAG recalls its experience and reconfigures the network without using the fault reconfiguration algorithm. This is the main step, GAG employs in reducing the execution time of fault reconfiguration. The GAG and its functionalities are described in Figure 5-2.

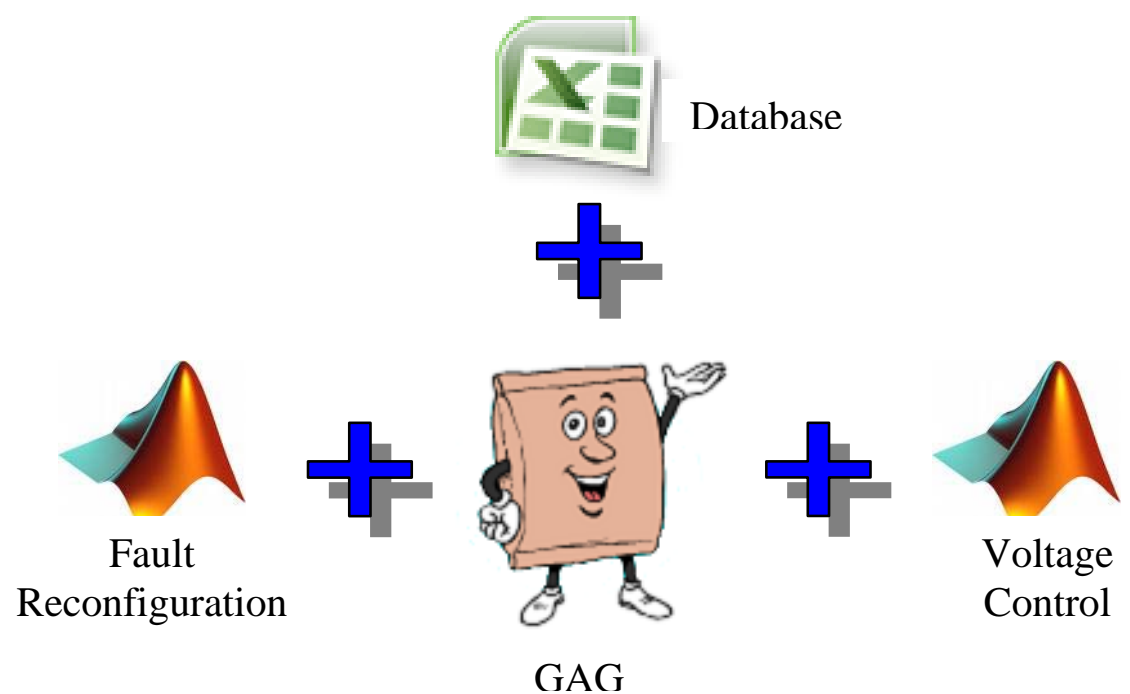

Figure 5-2: Global Agent (GAG) functionalities. 


\subsection{Circuit of the Future (CoF)}

The fault detection and reconfiguration algorithms are tested on the proto-type distribution system, the Circuit of the Future (CoF). This is the distribution system modeled and designed by Southern California Edison (SCE). The same distribution system is modeled using Power World Simulator and DEW. The modeled circuit using DEW is shown in Figure 5-3.

The CoF has two sources, one is the substation and the other is a distributed generator (DG). The substation is connected with three outgoing feeders, which will serve the loads in the distribution system. This system has 7 switches/disconnectors for the purpose of reconfiguration of faulty network. The distribution network has 14 loads demanding 26.69 MW of real power and 14.578 MVar of reactive power. The network has 14 shunt capacitor banks for the purpose of voltage control. It has two DGs, one for real power generation and other for reactive power generation.

For simplicity purposes the original circuit has been modified by lumping all the loads together without affecting the basic topology and key features of the original CoF circuit. This distribution network from now on will be termed as Simplified CoF. This Simplified CoF has been modeled in Power World Simulator, and in shown in Figure 5-4. The key components of Simplified CoF are listed below,

- 18 Buses

- 17 Distribution lines

- 7 Switchable Transmission lines (Switches/disconnectors)

- 11 fixed loads ( P: 26.69 MW, Q:14.578 MVAR, modeled as 'PQ buses')

- $1 \mathrm{DG}$ (P: $3 \mathrm{MW}$, modeled as 'PV bus')

- 1 Substation (Modeled as 'Slack Bus')

- 7 shunt capacitor banks (4.5 MVAR) 


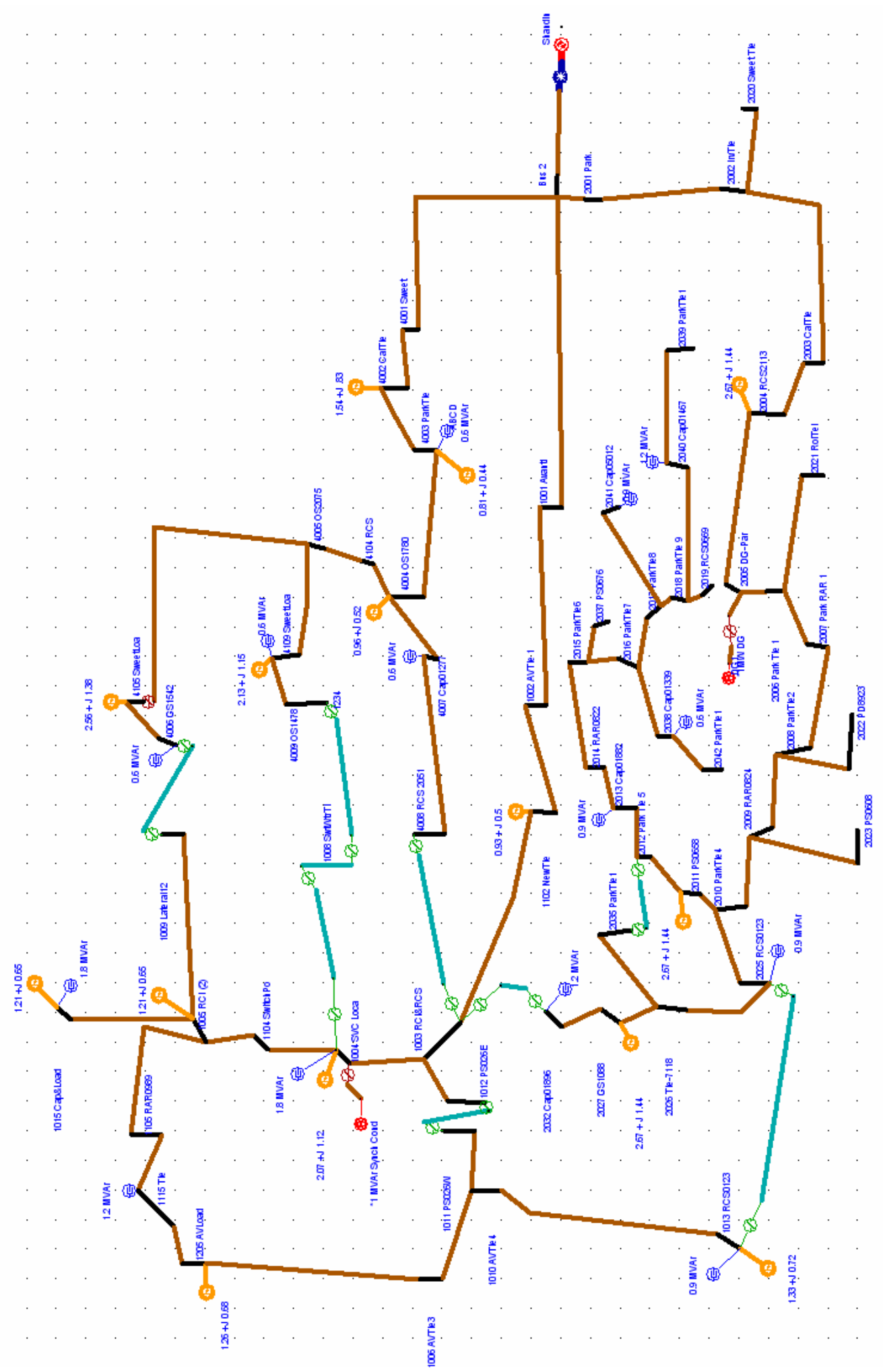

Figure 5-3: The Circuit of the Future modeled in DEW [2]. 
The 11 loads, which exist in the Simplified COF, have load priorities depending upon the importance of particular load. Loads such as Hospital loads and Industrial loads are given higher priority than any residential loads in the system. This distinction of the loads is necessary in the load shedding process for voltage control. The loads with lower priority will get shed before the higher priority loads. There are three higher priority loads $\left(L_{3}, L_{8}\right.$ and $\left.L_{10}\right)$, three lowest priority loads $\left(L_{7}, L_{9}\right.$ and $\left.L_{11}\right)$ and five middle priority loads $\left(L_{1}, L_{2}, L_{4}\right.$ $L_{5}$ and $L_{6}$ ) in the network.

\section{Simplified Circuit of the Future}

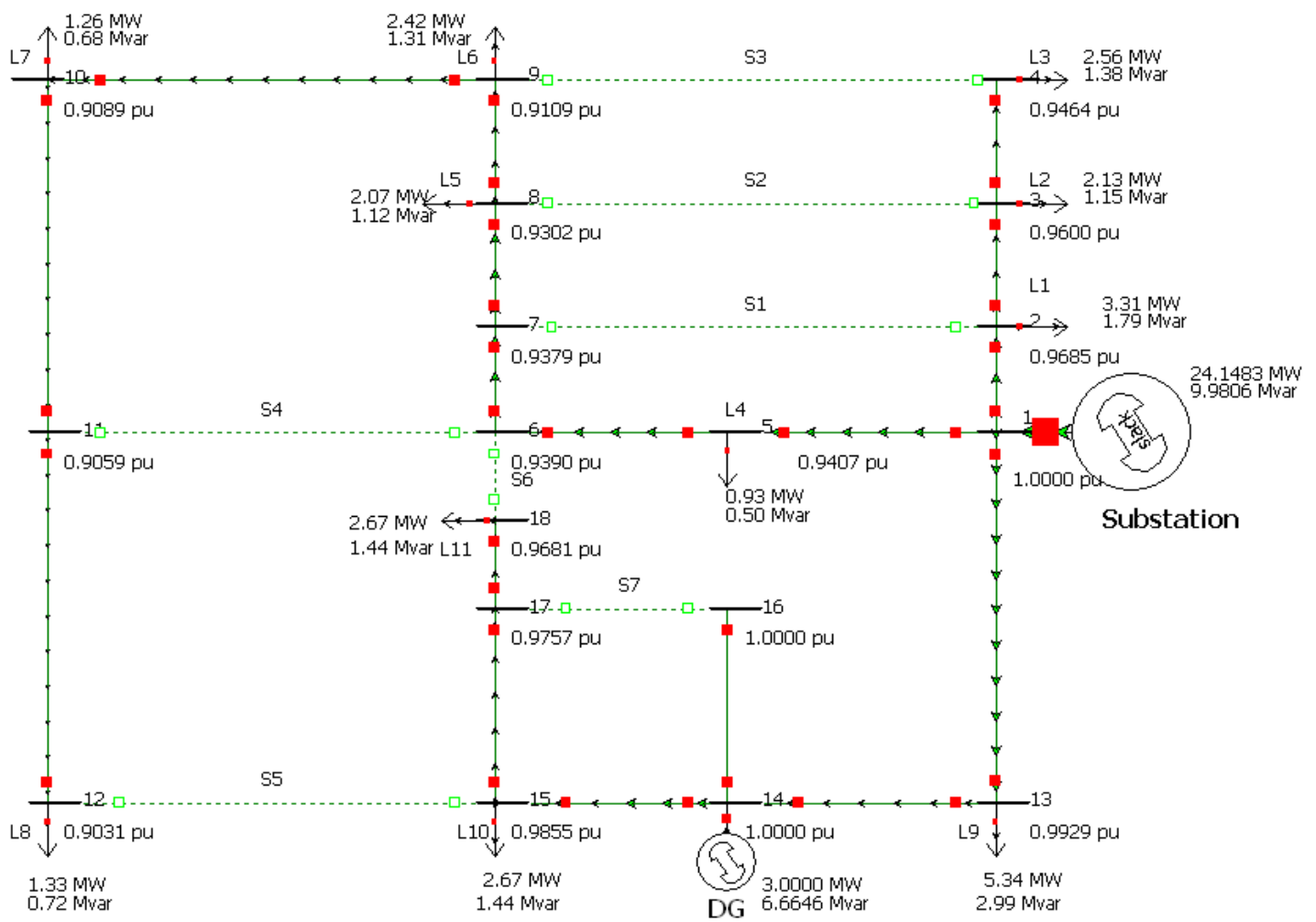

Figure 5-4: The Simplified CoF modeled in Power World Simulator. 


\subsection{Fault Detection and Reconfiguration Algorithm}

As discussed earlier fault detection is done by Load Agents (LAGs) and reconfiguration is achieved by Global agent (GAG). LAGs will follow the fault detection algorithm explained in section 4.2 , for fault detection. There are two main ways in which we can instantiate the agents.

- Instantiating JADE agents from reading the power flow report, which is generated by Power World Simulator.

- Instantiating JADE agents by passing necessary command line arguments to the JADE platform.

Both cases are efficient and correct as per the results are concerned. The only difference between them is, generating the command line arguments needed to instantiate the JADE agents.

The first step towards the simulation process is to simulate the fault in Power World Simulator. Once the fault is simulated, the corresponding load flow report is sent to Microsoft Excel. This excel file is then read by JADE function for instantiating the JADE agents. Once the LAGs, SAGs and GAG are created in the JADE platform, the fault is detected by LAGs and SAGs. The restoration needed for isolating the faulty part of the network and supplying the remaining part of the network is achieved by Global agent (GAG). The whole process is explained through the following flowchart, 


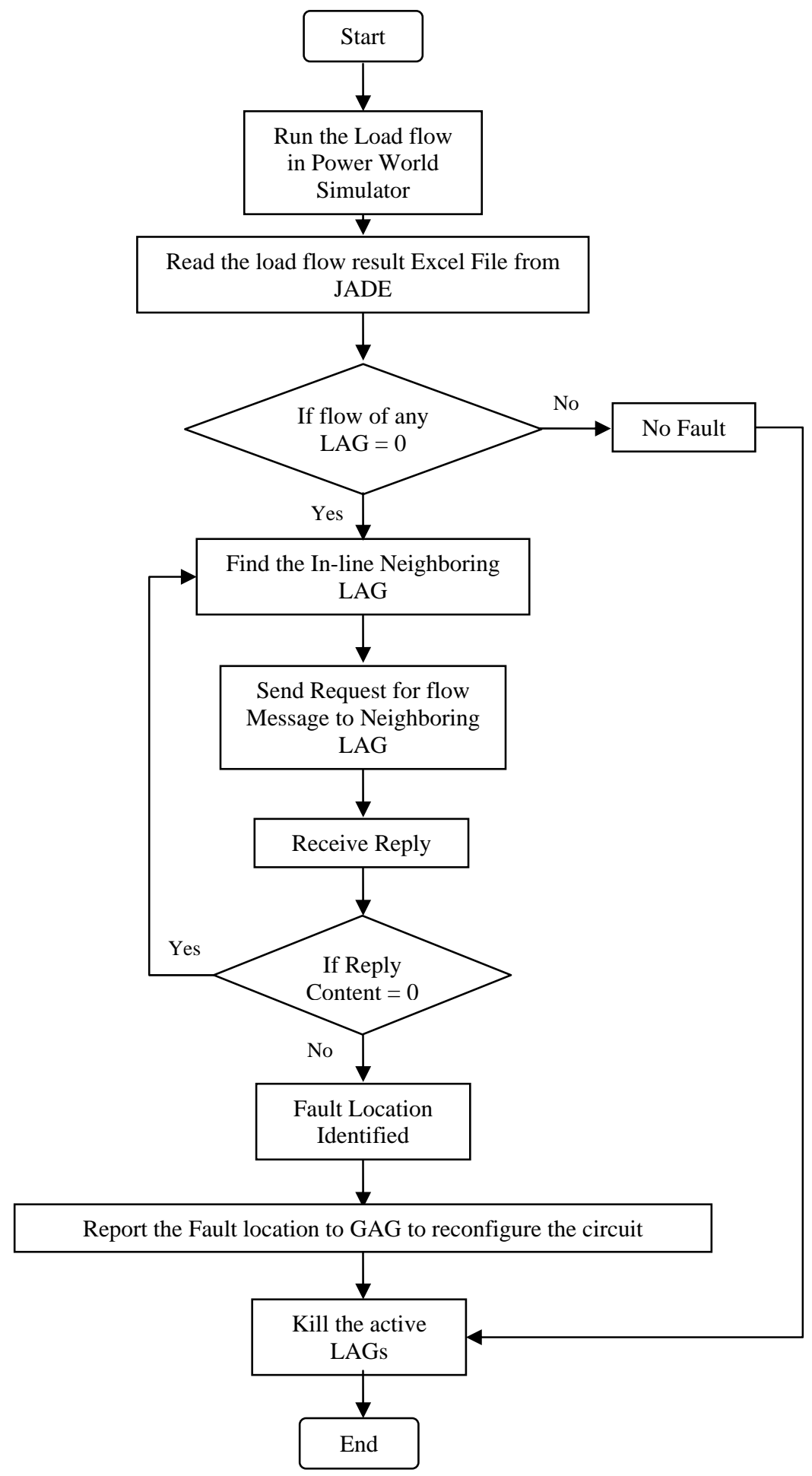

Figure 5-5: Flow chart for Fault Detection and Reconfiguration. 


\subsubsection{Test Case 1: For a Single Fault}

Let us test the system for a single fault in line $\mathrm{N}_{2} \mathrm{~N}_{3}$ in Feeder 1 . First of all, the fault is simulated in Power World Simulator and the corresponding network is depicted in Figure $5-6$.

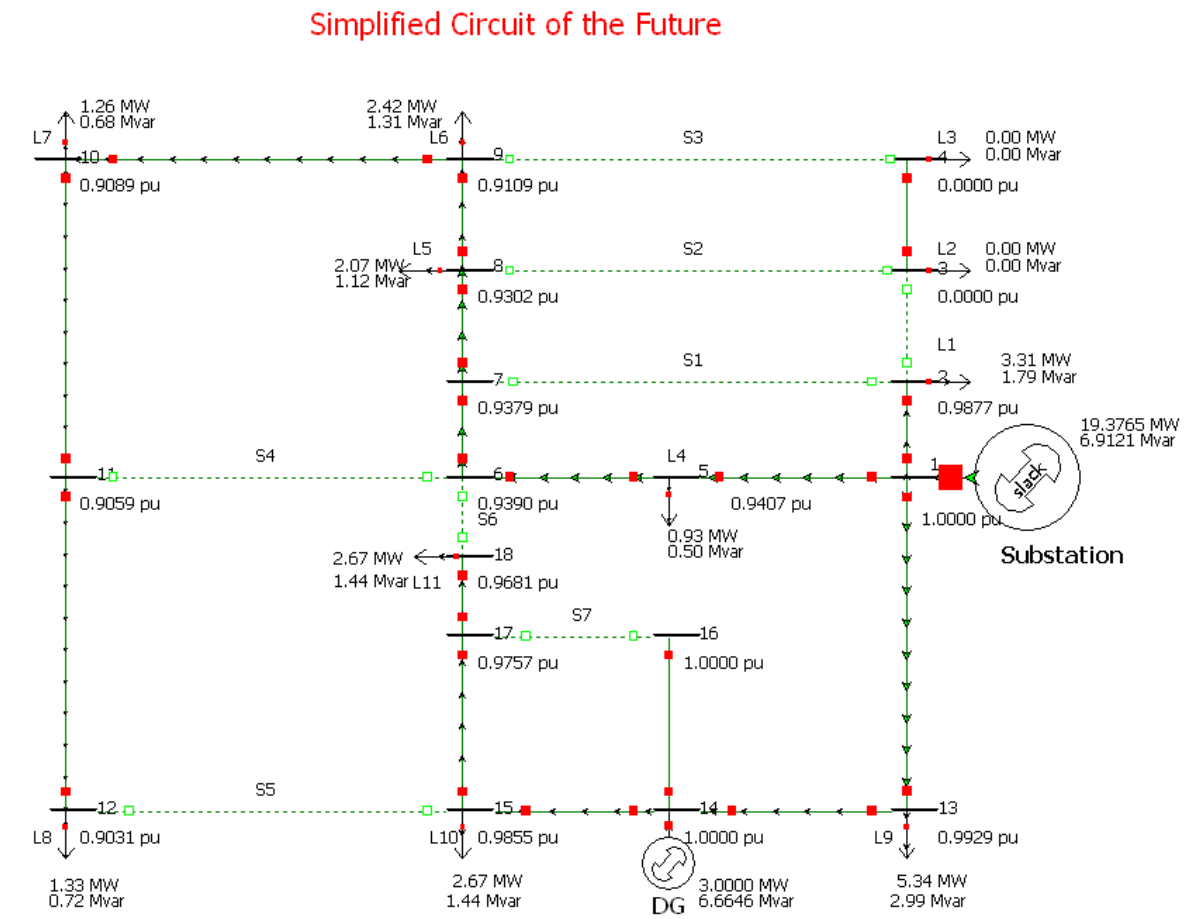

Figure 5-6: Simplified CoF with fault in line 2-3.

Once the fault is simulated in Power World Simulator, It produces following load flow report which is saved in excel file as shown below in Figure 5-7. This report has the power flow values of all loads. Because of the line fault at line 2-3, the loads $L_{2}$ and $L_{3}$ are not supplied at all. The same information can be observed from the following excel sheet, where MW values of ID: 2 and ID: 3 are zero. 


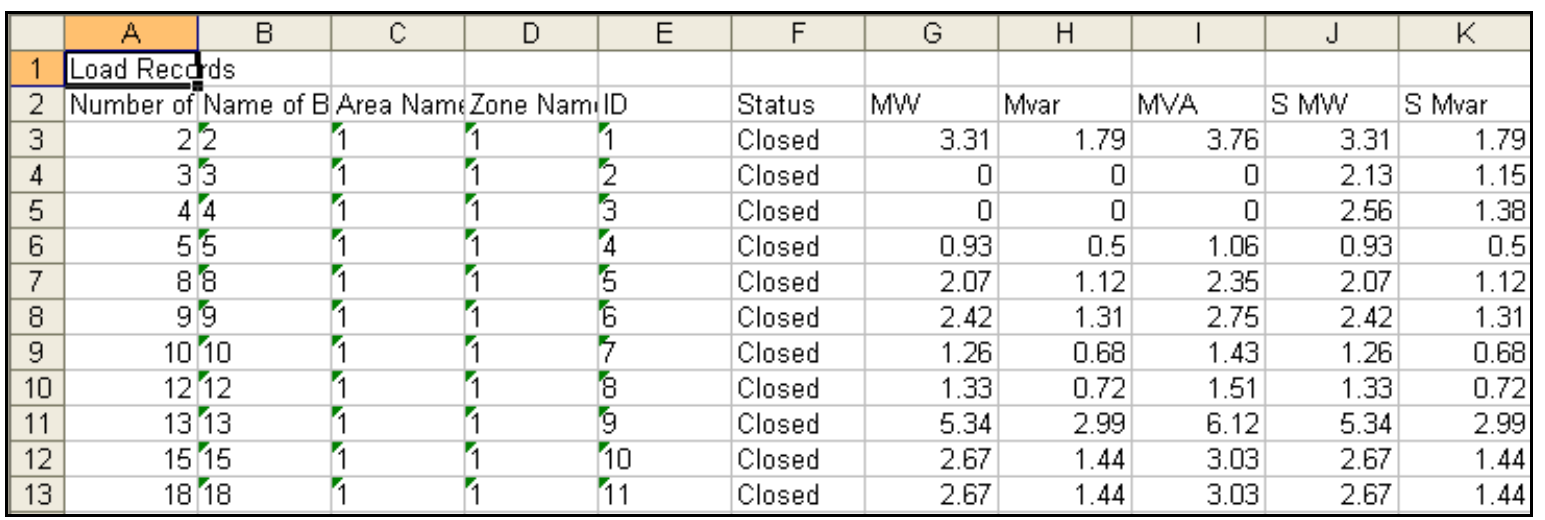

Figure 5-7: Power World Simulator's Load flow report for line fault at line 2-3.

When JADE MAS is run, the load agents in the faulty feeder will only get created. These agents will communicate and coordinate with each other to find out the fault location. The message passing between agents is clearly shown in JADE sniffer agent GUI.

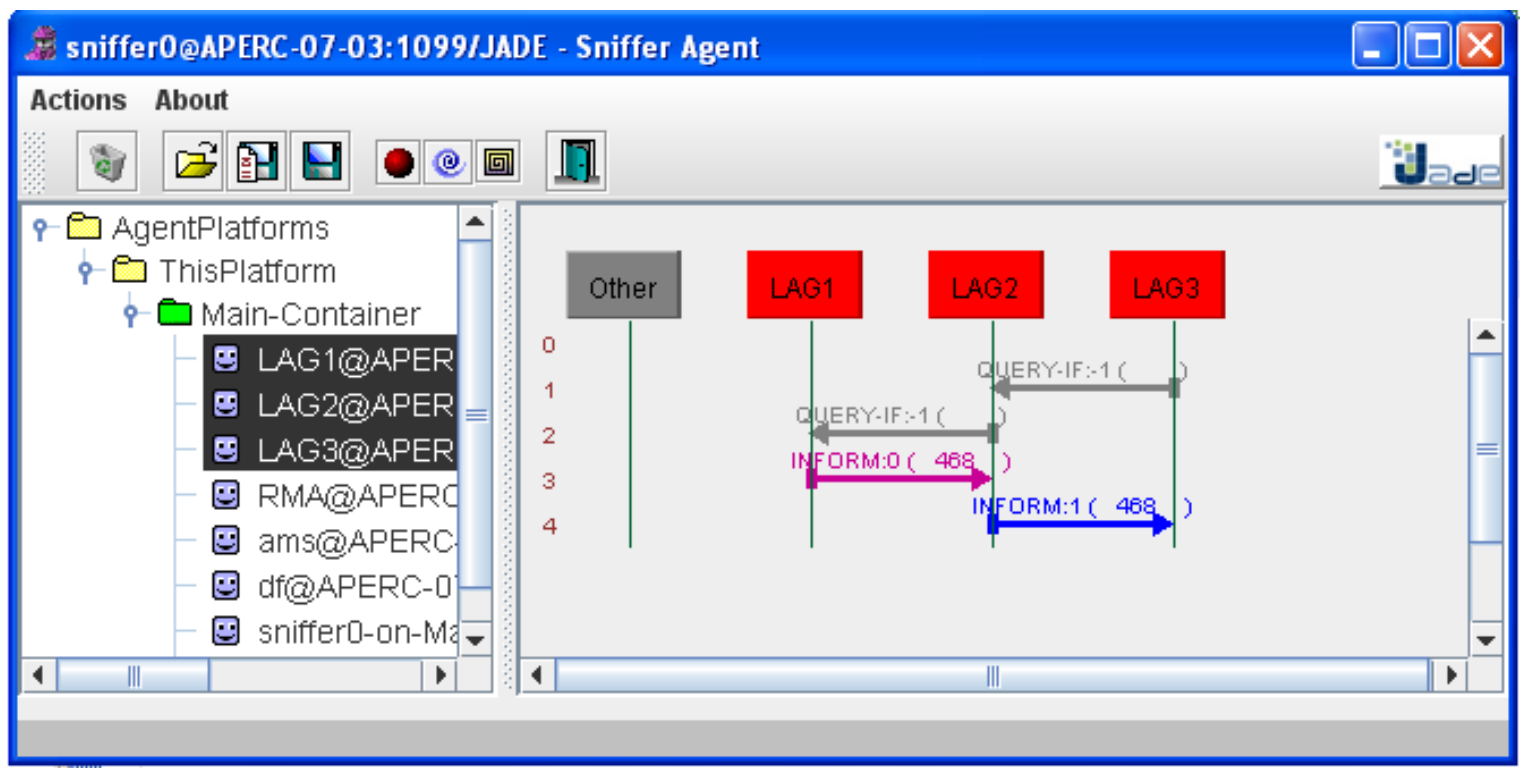

Figure 5-8: JADE message passing between agents for line fault at line 2-3. 
All the LAGs will be created in the particular feeder, but only those agents which sense zero power start communicating with their incoming neighboring load agents regarding whether they have power or not. In this particular scenario, the load agents LAG2 and LAG3 has no power. Once they sense zero power, they start sending the request message to their neighboring load agents, to LAG1 and LAG2 respectively. Here LAG1 will report its power flow value to LAG2, and LAG2 will report to LAG3. According to the fault detection algorithm explained in section 4.2 , the fault is detected at line 2-3, i.e. between nodes $\mathrm{N}_{2} \mathrm{~N}_{3}$. This solution can be found on Java output console, as shown in Figure 5-9.

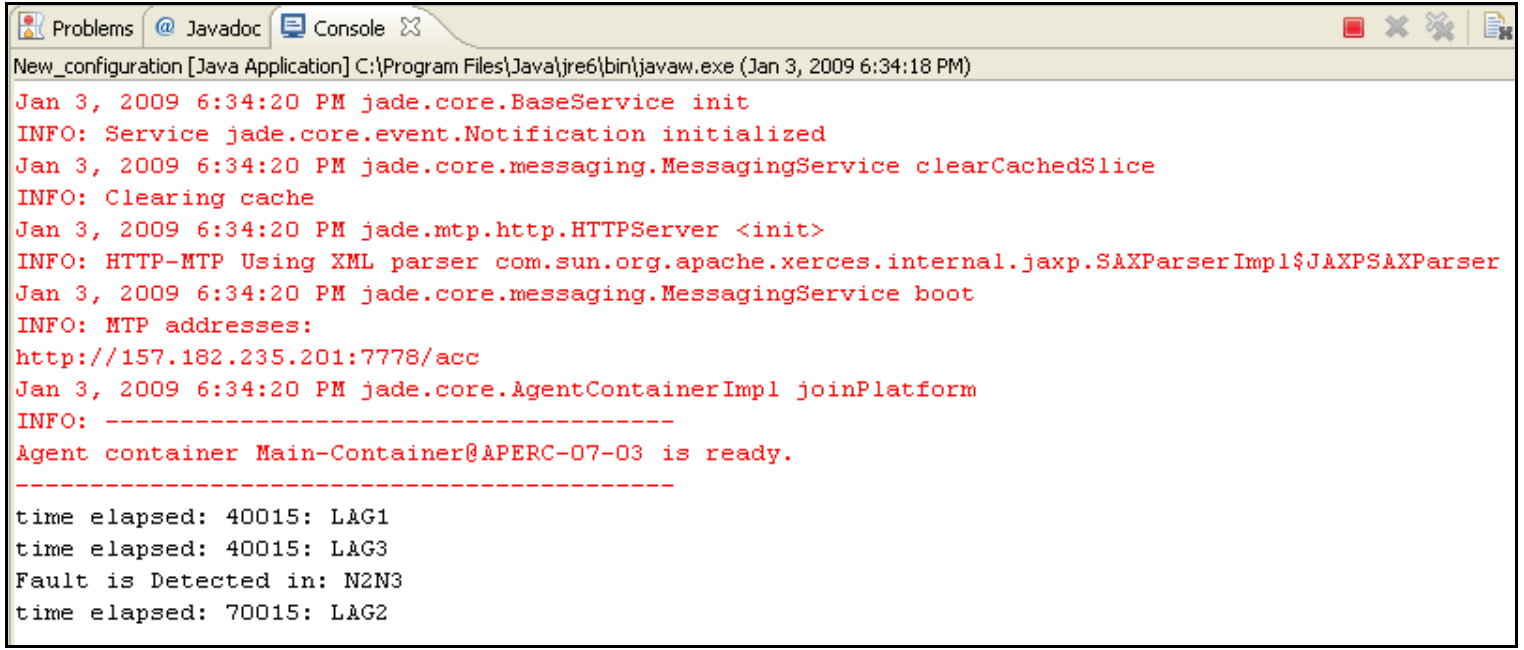

Figure 5-9: Java output console showing results of Test Case 1.

This fault location is now reported to Global Agent, which calls the MATLAB reconfiguration algorithm to reconfigure the network. The MATLAB reconfiguration algorithm is explained in section 4.3. Once the algorithm finds the reconfigured network, it plots the network and prints the results. The reconfigured network for fault at line 2-3 is shown in following Figure 5-10. 

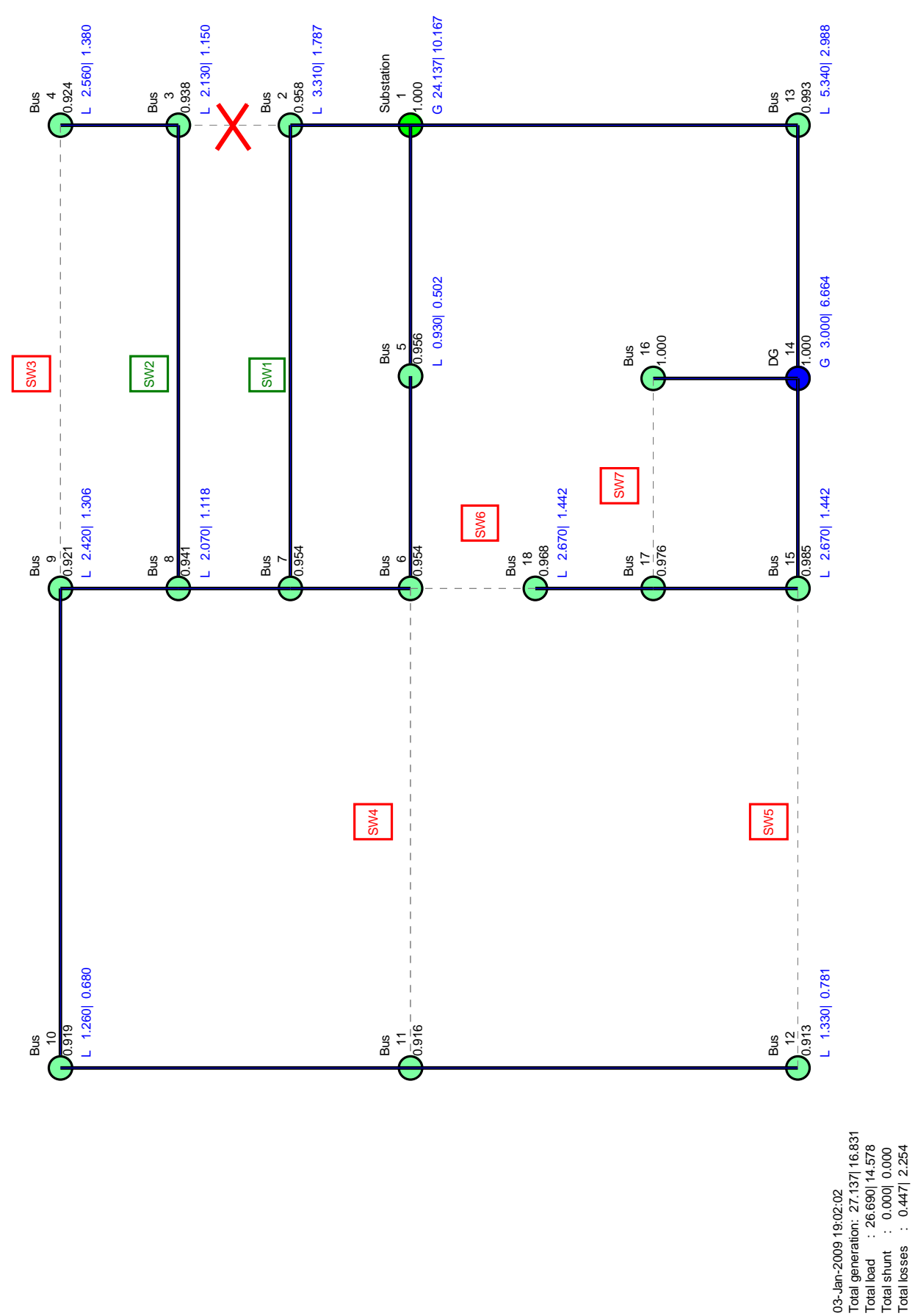

Figure 5-10: MATLAB plot depicting reconfiguration network for fault at line 2-3. 


\subsubsection{Test Case 2: For Multiple Faults}

Let us assume that there are two faults in Feeder 1 and Feeder 2 at $N_{1} N_{2}$ and $N_{1} N_{5}$. The procedure would be as same as in the previous test case. This scenario is been simulated first in Power World Simulator and the respective command line arguments are sent to JADE platform to instantiate the agents. The message passing between the Load Agents (LAGs) have been observed and shown in following figure.

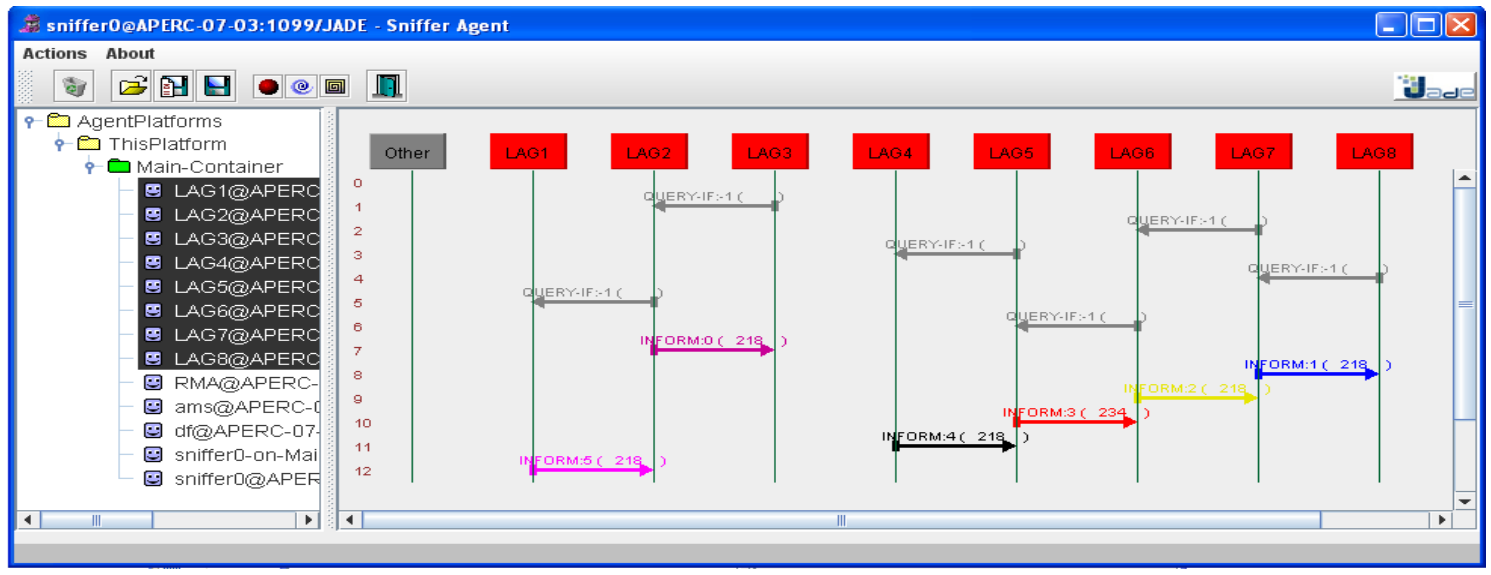

Figure 5-11: JADE message passing between agents for line faults at line 1-2 and 1-5.

The fault is detected according to the fault detection algorithm and shown on output Java console as shown below,

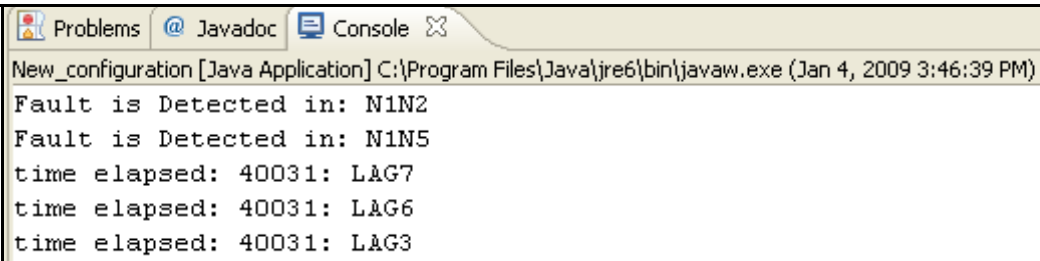

Figure 5-12: Java output console showing results of Test Case 2.

Once the faults are detected by LAGs, the information is then sent to GAG for reconfiguration. The reconfiguration network is shown in Figure 5-13. 

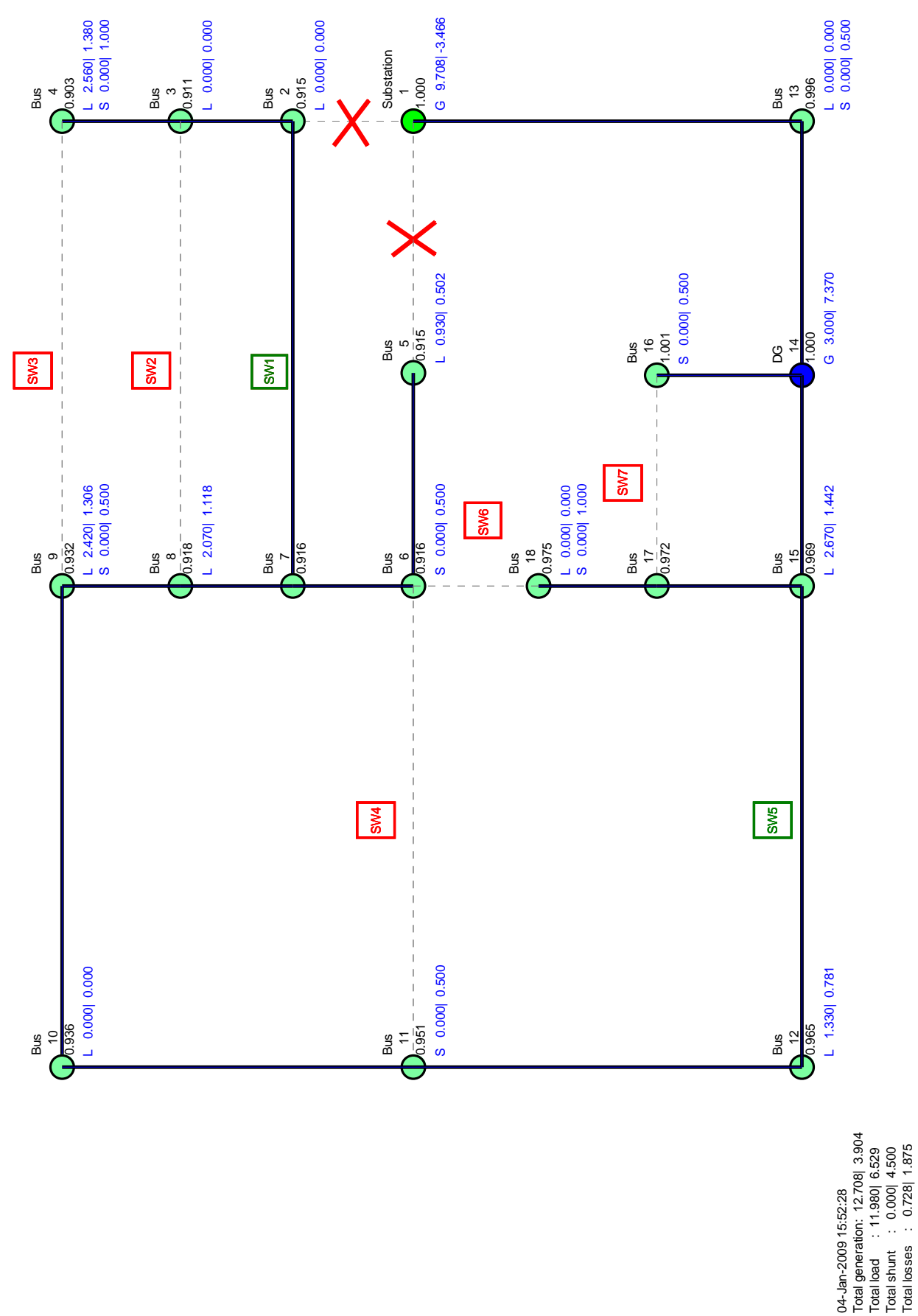

Figure 5-13: MATLAB plot depicting reconfiguration network for fault at line 1-2 and 1-5. 


\subsection{MATLAB Reconfiguration}

This section is mainly concentrated on MATLAB reconfiguration which is used by Global Agent (GAG) for reconfiguration of faulty network. Many key features of the fault reconfiguration algorithm are explained with several scenarios. A Graphical User Interface (GUI) is developed for easy user interaction with the fault reconfiguration algorithm and to carry out the simulations. The GUI is shown in the following figure.

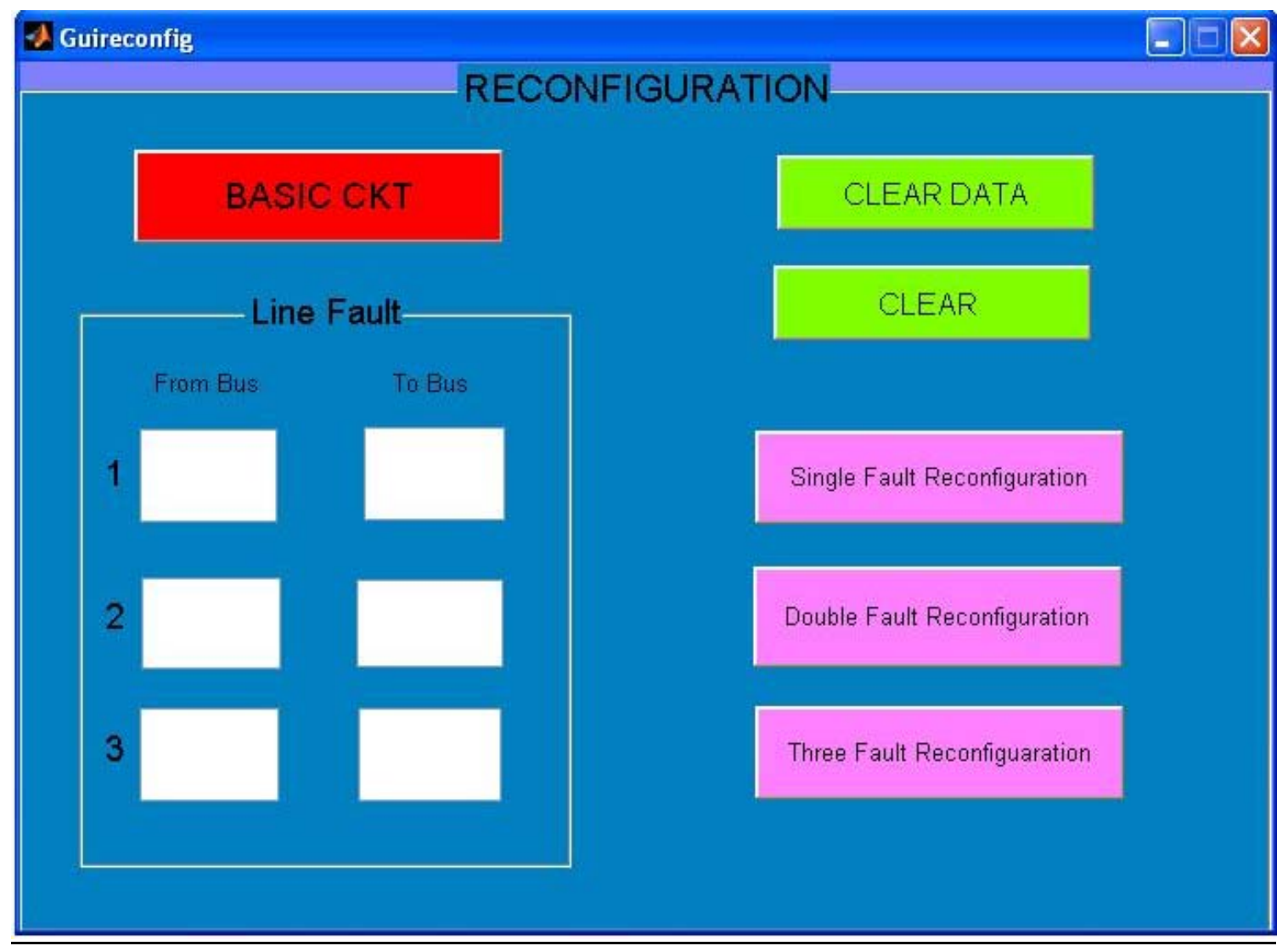

Figure 5-14: MATLAB GUI for Fault Reconfiguration. 
The basic functionalities of the above GUI are explained below,

- BASIC CKT: This push button will display the basic network configuration of Simplified CoF.

- Line Fault: The user can specify up to three faults and create the faults in the system.

- CLEAR DATA: Clears the data in the Line fault section.

- CLEAR: Clears the MATLAB command prompt.

- Single Fault Reconfiguration: Reconfigures the circuit based on fault location specified in Line Fault 1 section.

- Double Fault Reconfiguration: Reconfigures the circuit based on fault locations specified in Line Fault 1 and Line Fault 2 sections.

- Three Fault Reconfiguration: Reconfigures the circuit based on fault locations specified in Line Fault 1, Line Fault 2 and Line Fault 3.

The basic Simplified CoF network is shown in the Figure 5-15. In this plot each and every bus is marked with its bus number and its voltage magnitude. The loads are marked with their real and reactive power consumptions. In the same way generators are marked with read and reactive power generations. The switches are represented with red rectangular boxes when they are open and turn in to green when they are closed. In the bottom left corner of the plot, information related to total generation, total load and total losses in the system are shown. Any transmission line which is in normal operation is shown with solid line, where as transmission line which is opened, is shown with dashed line. 


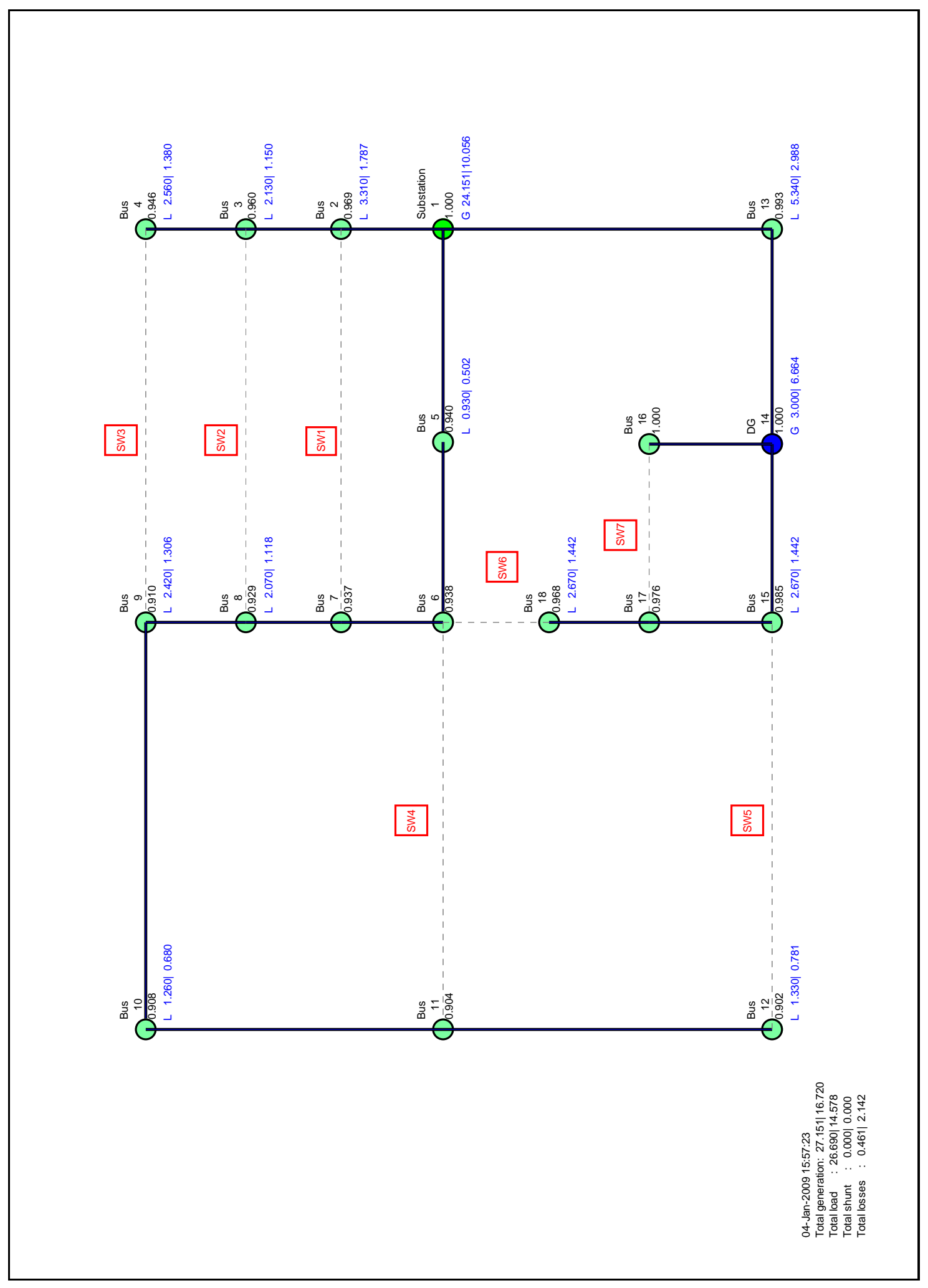

Figure 5-15: MATLAB plot depicting basic Simplified CoF. 


\subsubsection{Test Case 3: For a Line Fault}

Let us assume that the fault is at line 1-2. In order to simulate this fault, one has to enter the 'From bus' and 'To bus' as 1 and 2 in the reconfiguration GUI line fault section. Once these fields are entered the reconfiguration can be achieved using fault reconfiguration algorithm as explained in the section 4.3. The obtained reconfiguration circuit would satisfy all the objectives and constraints specified in section 4.3.4.

The reconfiguration algorithm execution key steps are explained as below,

1. When there is a fault at line 1-2, the possible single switch combinations for reconfiguring the network according to fault reconfiguration algorithm would be,

$$
S\left(e_{f}\right)=\left\{S_{1}, S_{2}, S_{3}\right\}
$$

The algorithm first tries closing the switches $S_{1}, S_{2}$, and, $S_{3}$ individually, and the voltage profiles for each combination would be shown as below,

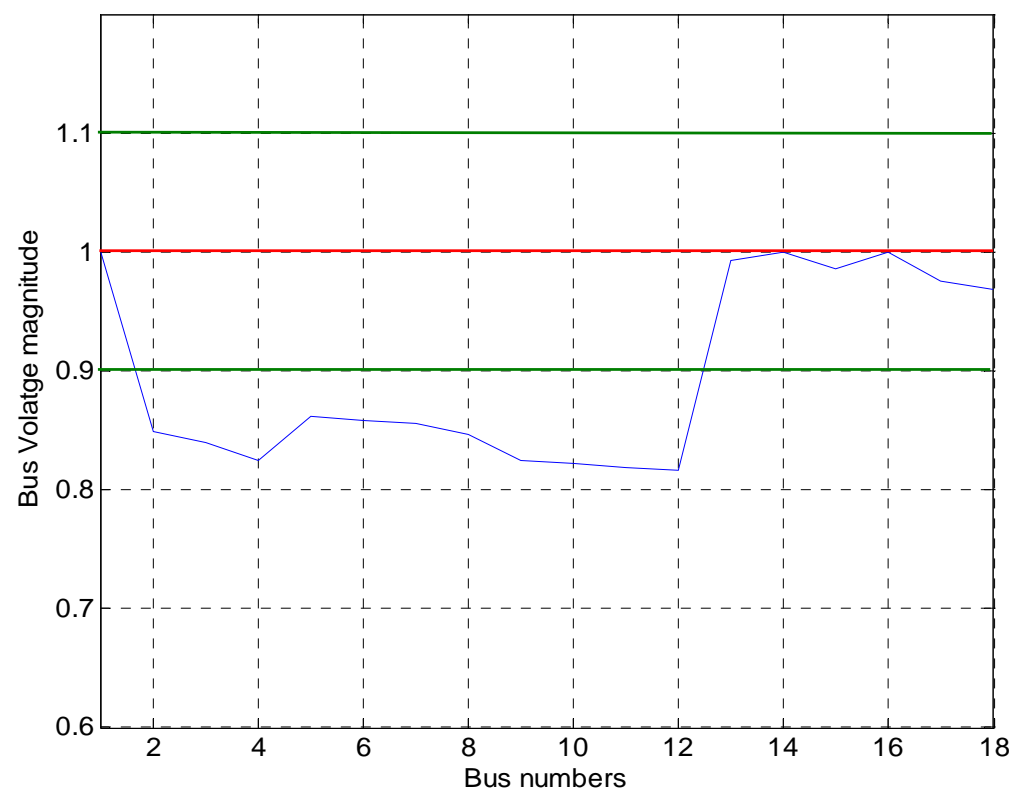

Figure 5-16: MATLAB plot depicting voltage profile with $\mathrm{S}_{1}$ closed for line fault at 1-2. 


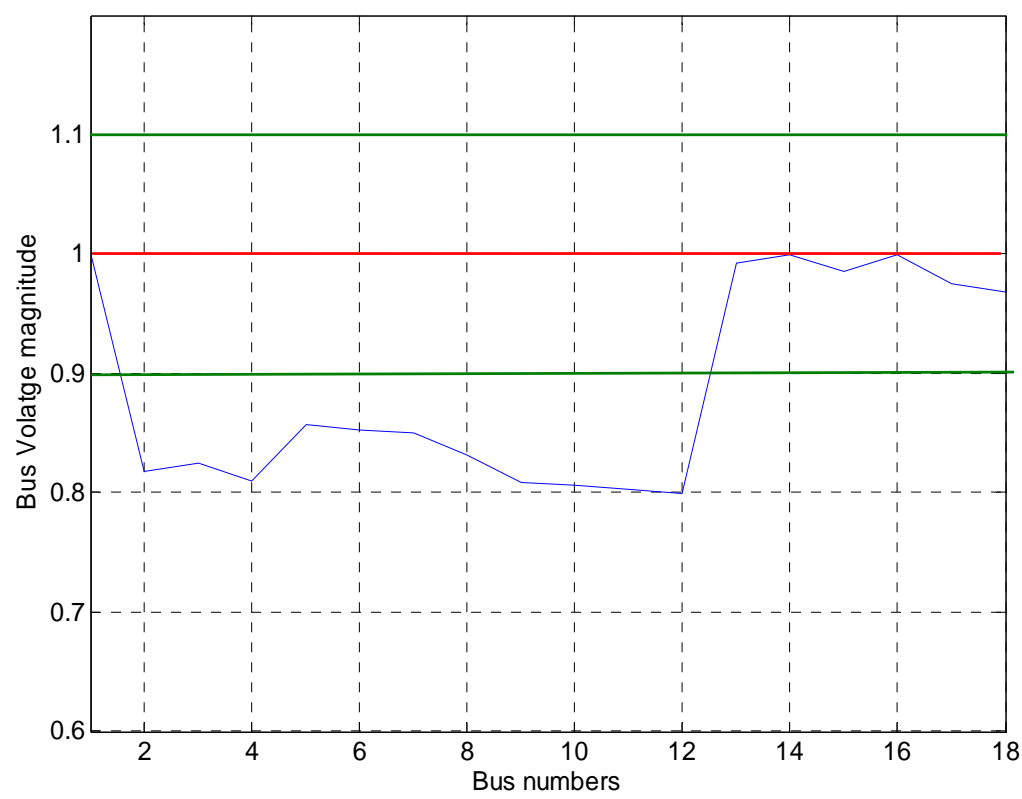

Figure 5-17: MATLAB plot depicting voltage profile with $S_{2}$ closed for line fault at 1-2.

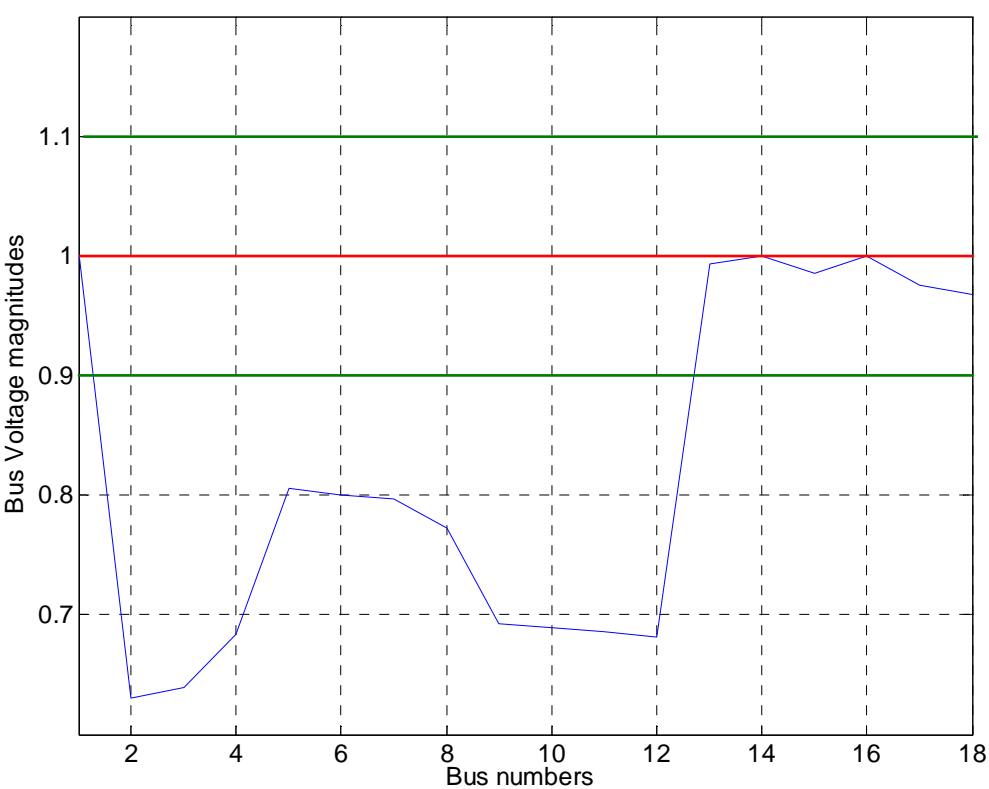

Figure 5-18: MATLAB plot depicting voltage profile with $S_{3}$ closed for line fault at 1-2. 
2. From the above figures Figure 5-16, Figure 5-17 and Figure 5-18, one can observe that none of the individual switch configuration gives the good voltage profile, i.e. voltage magnitude with $\pm 10 \%$ tolerance. Now the algorithm searches for one more switch to operate by keeping the $S_{1}$ on, which can reconfigure the network with no voltage violation. The same procedure repeats with $S_{2}$ and $S_{3}$.

3. Finally, the fault reconfiguration algorithm finds two switch combinations which have no voltage violations, they are $\left\{S_{2}, S_{5}\right\}$ and $\left\{S_{1}, S_{5}\right\}$. The following figures show the voltage profile of the reconfiguration network with above switch configurations.

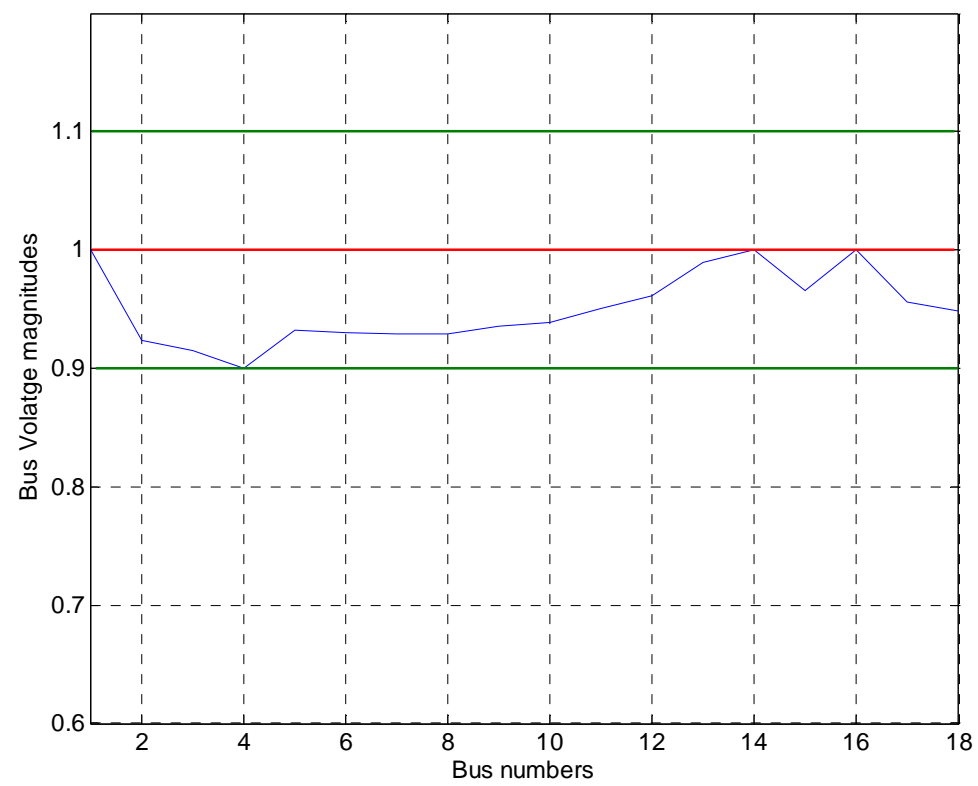

Figure 5-19: MATLAB plot depicting voltage profile with $S_{1} \& S_{5}$ closed for line fault at 1-2. 


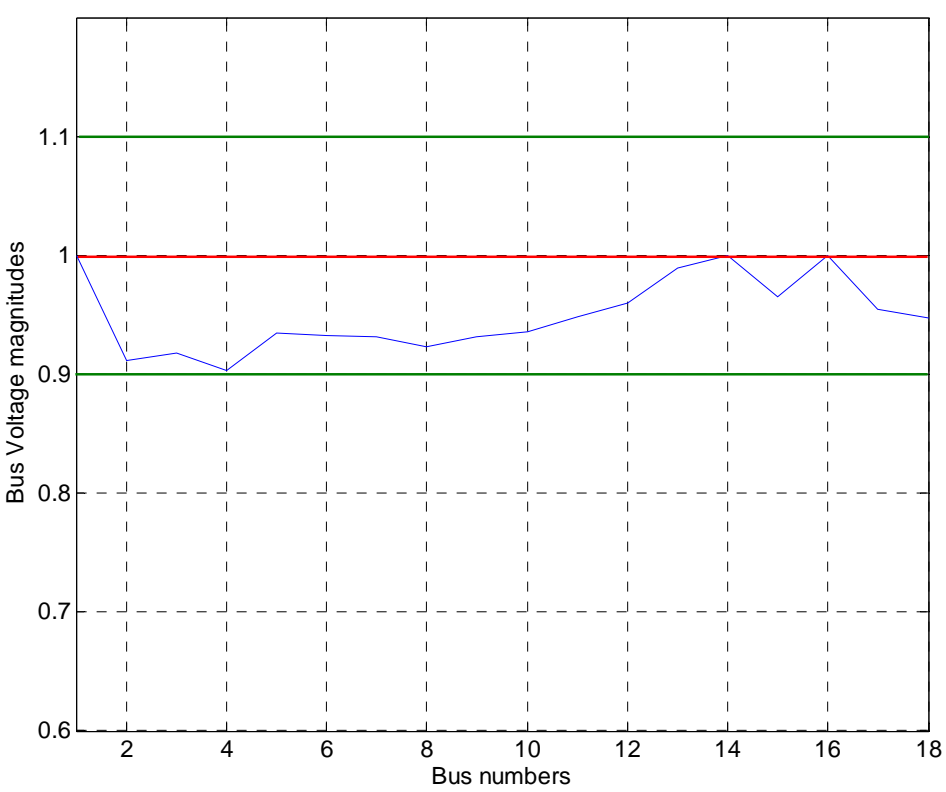

Figure 5-20: MATLAB plot depicting voltage profile with $S_{2} \& S_{5}$ closed for line fault at 1-2.

4. Hence there are two solutions for reconfiguring the line fault at 1-2. As explained in the section 4.3.4, whenever there are multiple solutions available for reconfiguration the algorithm chooses the configuration with less power loss. The reconfigured network with $\left\{S_{1}, S_{5}\right\}$ has $1.087 \mathrm{MW}$ real power loss where as the reconfigured network with $\left\{S_{2}, S_{5}\right\}$ has 1.151 MW of real power loss. Since, the reconfiguration with $\left\{S_{1}, S_{5}\right\}$ closed will yield less power loss, this configuration is selected as the final solution for reconfiguration. The final reconfigured network for line fault at 1-2 is shown in the following figure. 

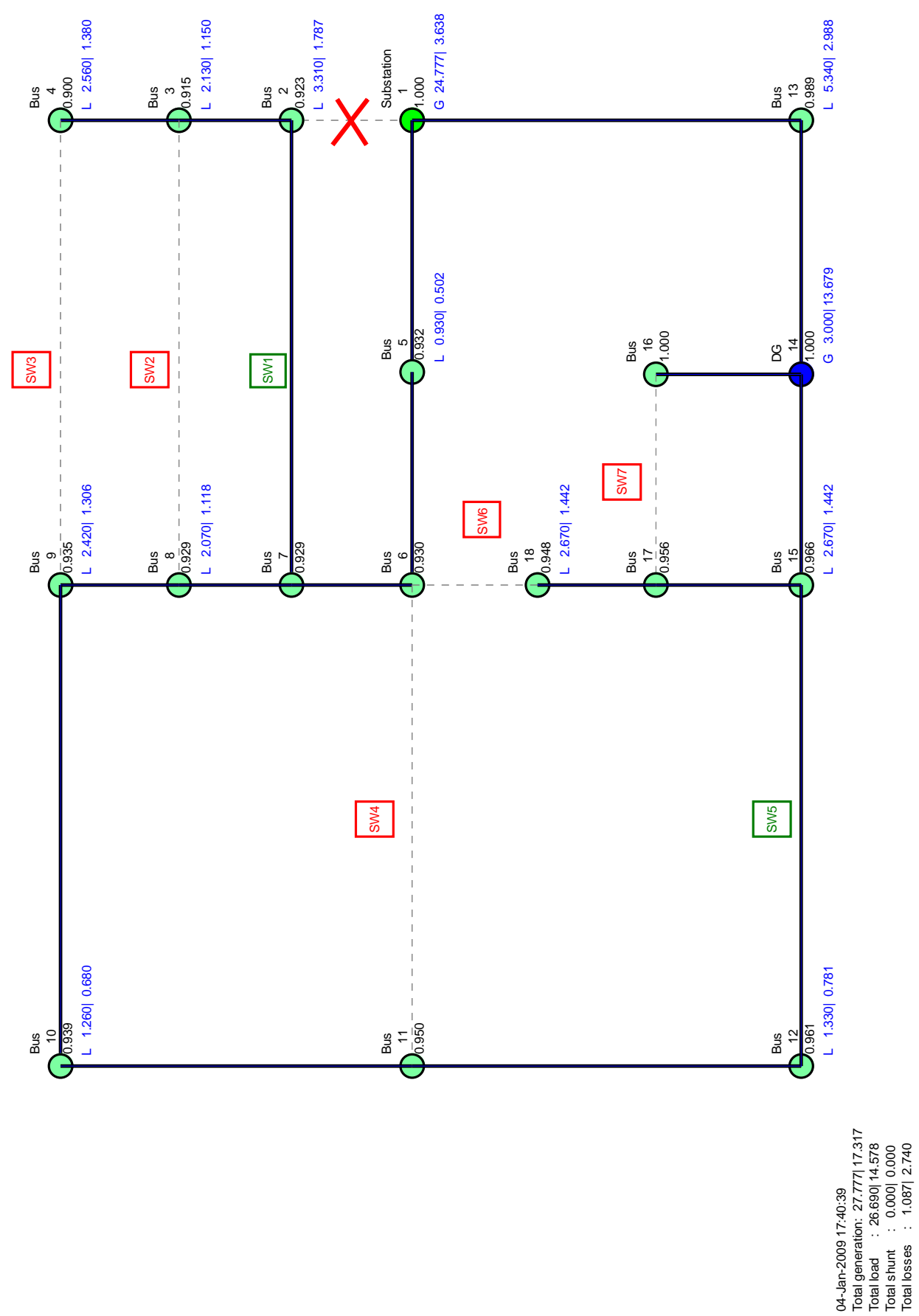

Figure 5-21: MATLAB plot depicting reconfiguration network for fault at line 1-2. 


\subsubsection{Test Case 4: For two Line Faults}

Let us assume that there are two faults at lines 1-2 and 14-15. The main aim of this scenario is to explain the voltage control part in Fault Reconfiguration algorithm. As explained before in the section 5.4.1, the algorithm tries searching for the best switch configuration in regards of voltage profile and power loss. But, in certain cases it is difficult to achieve the good voltage profile with only switch selection. In these types of cases the Fault Reconfiguration algorithm uses its voltage control routine to achieve good voltage profile for the reconfigured network.

In this scenario, the algorithm comes up with switch combination $\left\{S_{1}, S_{5}, S_{7}\right\}$, for reconfiguring the network. The voltage profile with this switch combination is shown in the following figure,

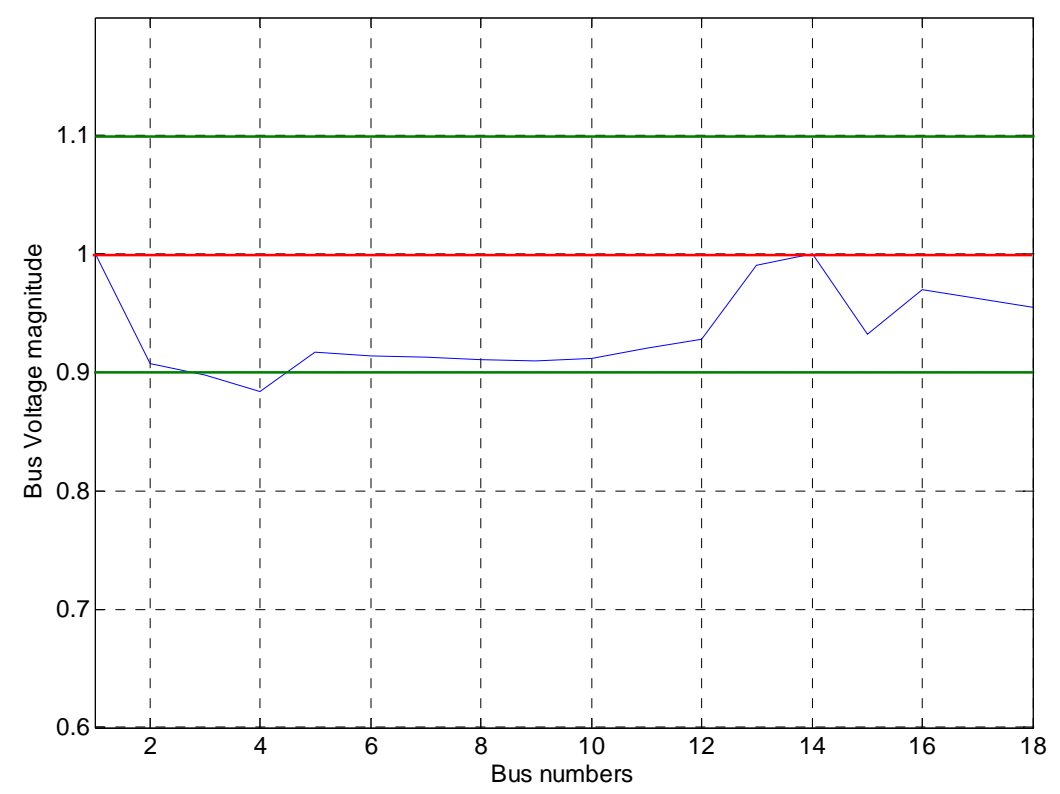

Figure 5-22: Plot depicting voltage profile with $S_{1}, S_{5} \& S_{7}$ closed for line faults at 1-2 \& 14-15, without voltage control. 
From the above figure it is clear that there is voltage violation in the reconfigured network. Now, the algorithm calls its voltage control routine to build a good voltage profile. This voltage control routine first tries putting all the shunt compensation available in the network. If the voltage violation still exists, it starts priority based shedding of the loads. In this scenario, when the shunt compensation of 4.5 MVAR is provided in the reconfigured circuit, the voltage violation disappears. The fact can be witnessed from following figure.

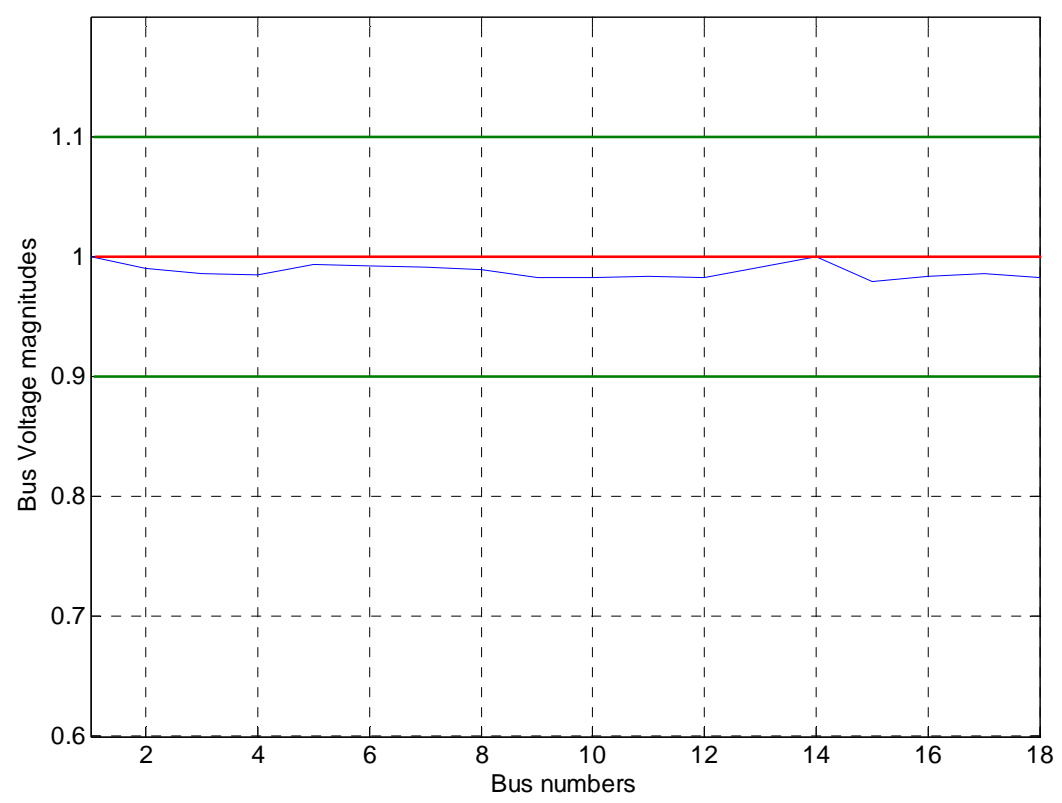

Figure 5-23: Plot depicting voltage profile with $S_{1}, S_{5} \& S_{7}$ closed for line faults at 1-2 \& 14-15 with shunt compensation.

The voltage profile in the above Figure 5-23 has no voltage violation. The shunt compensation of 4.5 MVAR helped in boosting up the bus voltages. The final reconfigured network is shown in following Figure 5-24. 


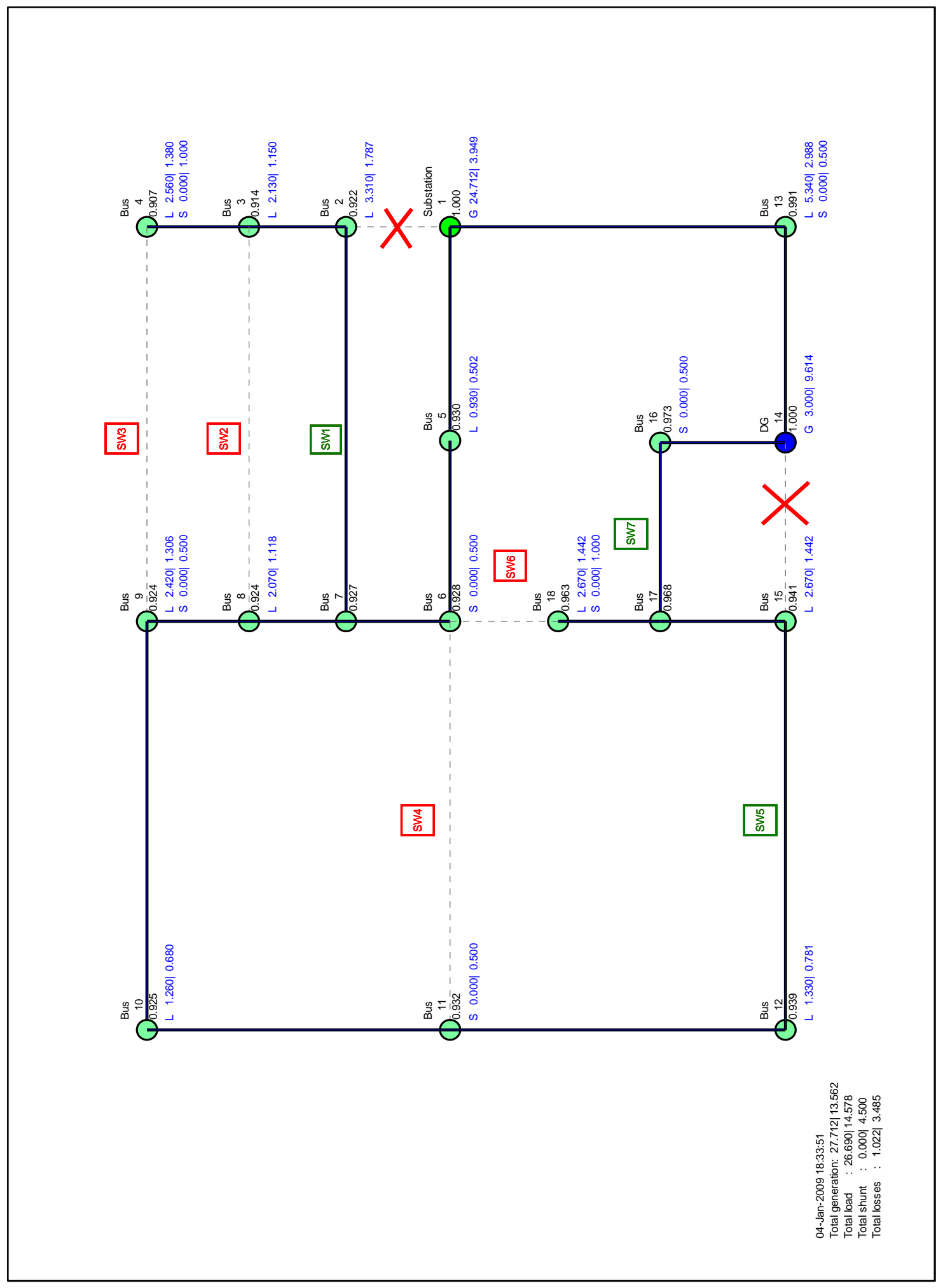

Figure 5-24: MATLAB plot depicting reconfiguration network for fault at lines 1-2 and 14-15. 


\subsubsection{Test Case 5: For three Line Faults}

Let us assume that there are three line faults at 1-2, 14-15, and 11-12. In this scenario, the algorithm comes up with switch combination $\left\{S_{1}, S_{5}, S_{7}\right\}$, for reconfiguring the network. The voltage profile with this switch combination is shown in the following figure,

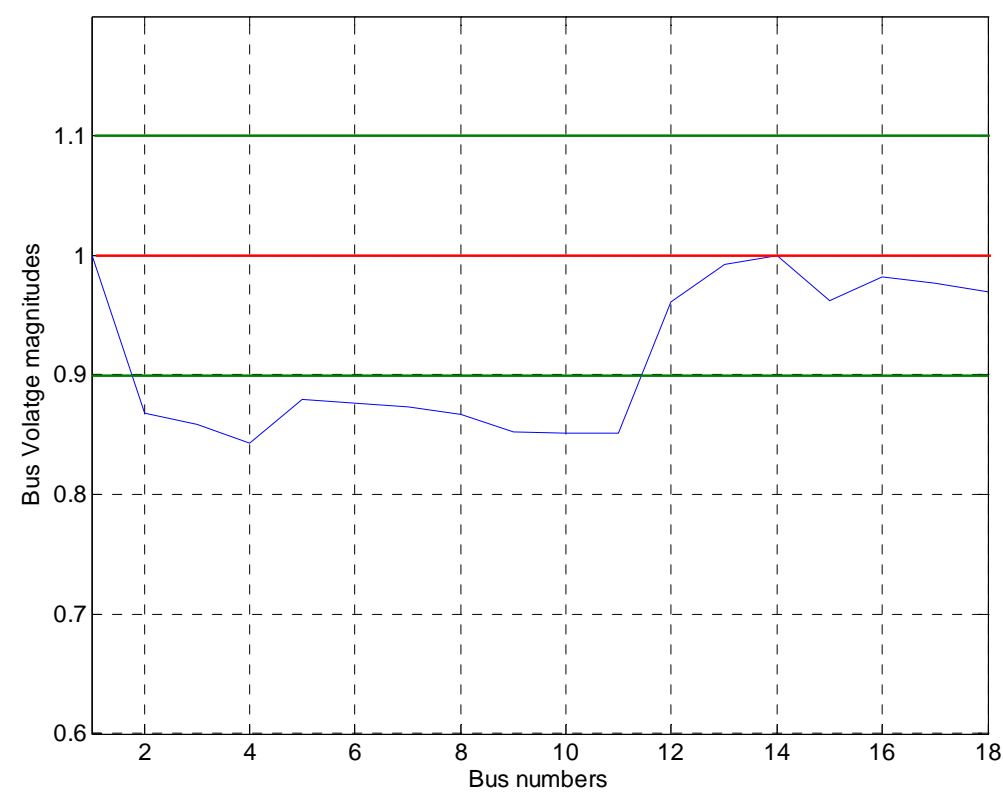

Figure 5-25: Plot depicting voltage profile with $S_{1}, S_{5} \& S_{7}$ closed for line faults at 1-2, 14-15 \& 11-12, without voltage control.

From the above figure it is clear that there is voltage violation. In this scenario, the algorithm calls the voltage control routine to achieve the good voltage profile. This time not only the shunt compensation but also the priority based load shedding is done for providing good voltage profile to the reconfigured network. The low priority loads such as, $L_{11}, L_{9}, L_{7} \& L_{1}$ are shed and shunt compensation of 4.5 MVAR are provided in order to achieve good voltage profile. The final reconfigured network is shown in the following figure. 


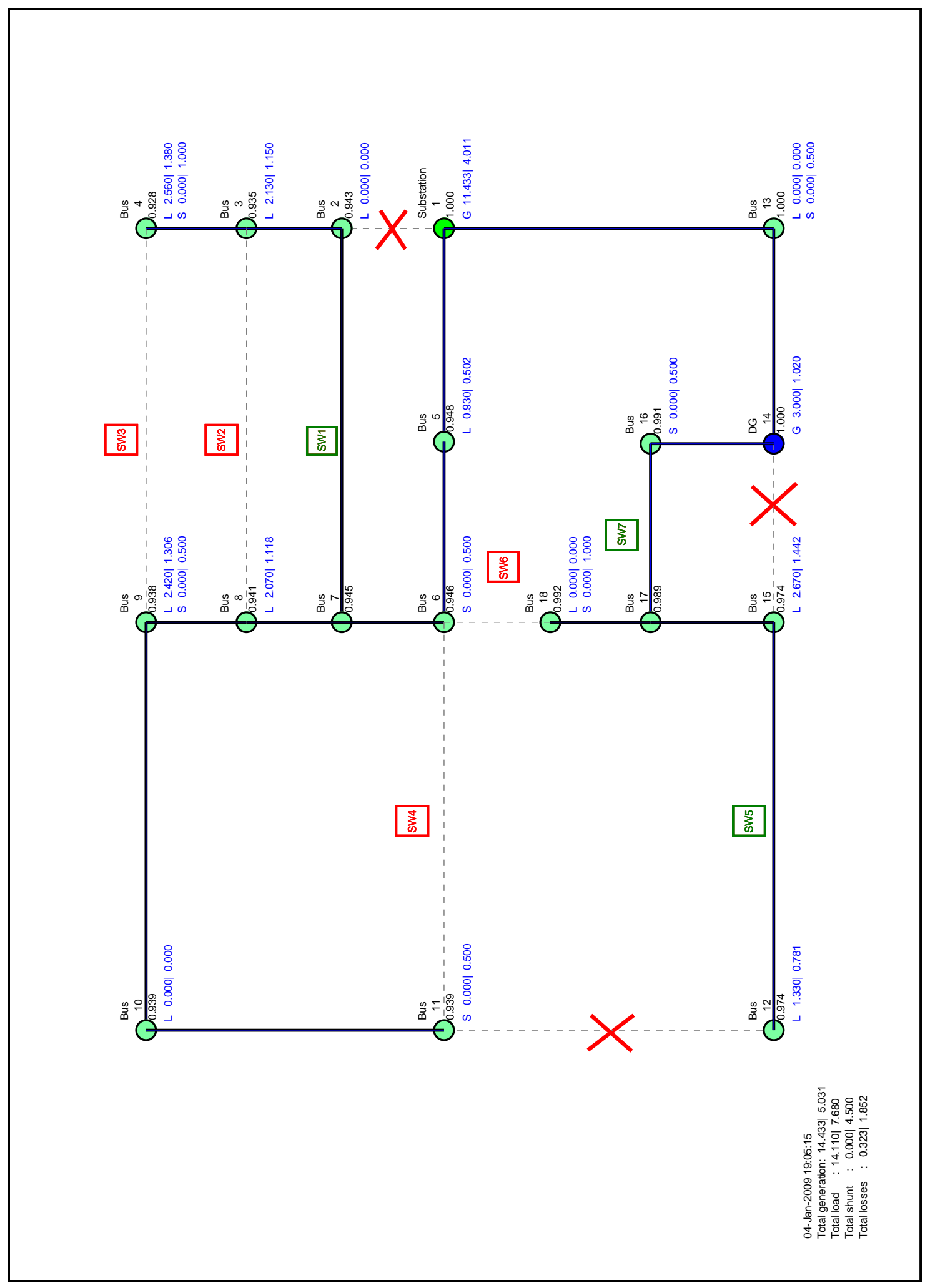

Figure 5-26: MATLAB plot depicting reconfiguration network for fault at lines 1-2, 14-15 and 11-12. 


\subsubsection{Test Case 6: Special Scenario}

In this section, a special scenario is discussed. Let us assume there are two faults at lines 9-10 and 10-11. In this particular scenario, there is no way that load $L_{7}$ is supplied. In these types of scenarios, the algorithm modifies its network data file in a manner to exclude the particular load which is not supplied. Once this has been done, the algorithm executes in a normal way to reconfigure the rest of the circuit. The MATLAB output command window for this scenario is shown in the following Figure 5-27,

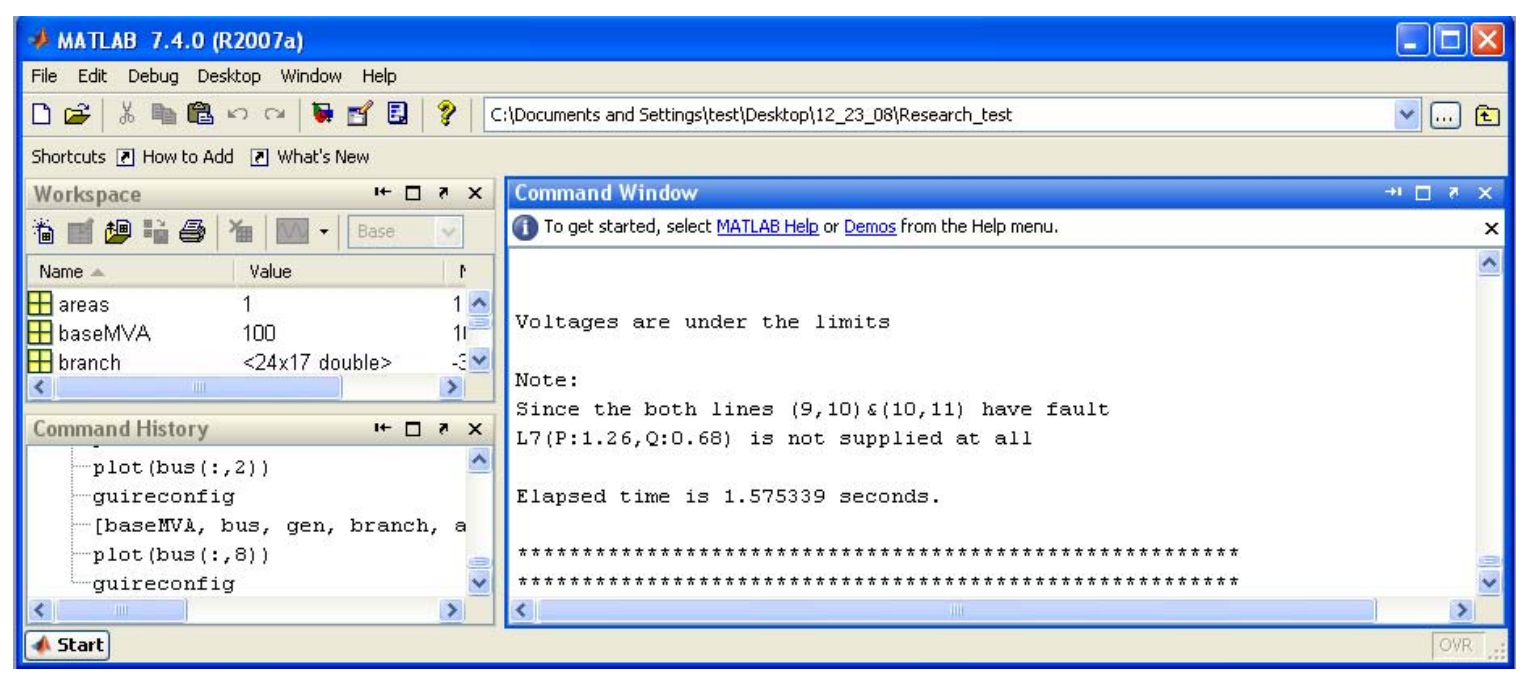

Figure 5-27: MATLAB output command window showing solution of Test Case 5.

The algorithm comes up with switch combination $S_{5}$ for reconfiguring the network with faults at 9-10 and 10-11. It can be observed from the above figure that there is no voltage violation in the network with switch configuration $S_{5}$. The final reconfigured network for this scenario is shown in the following Figure 5-28. 


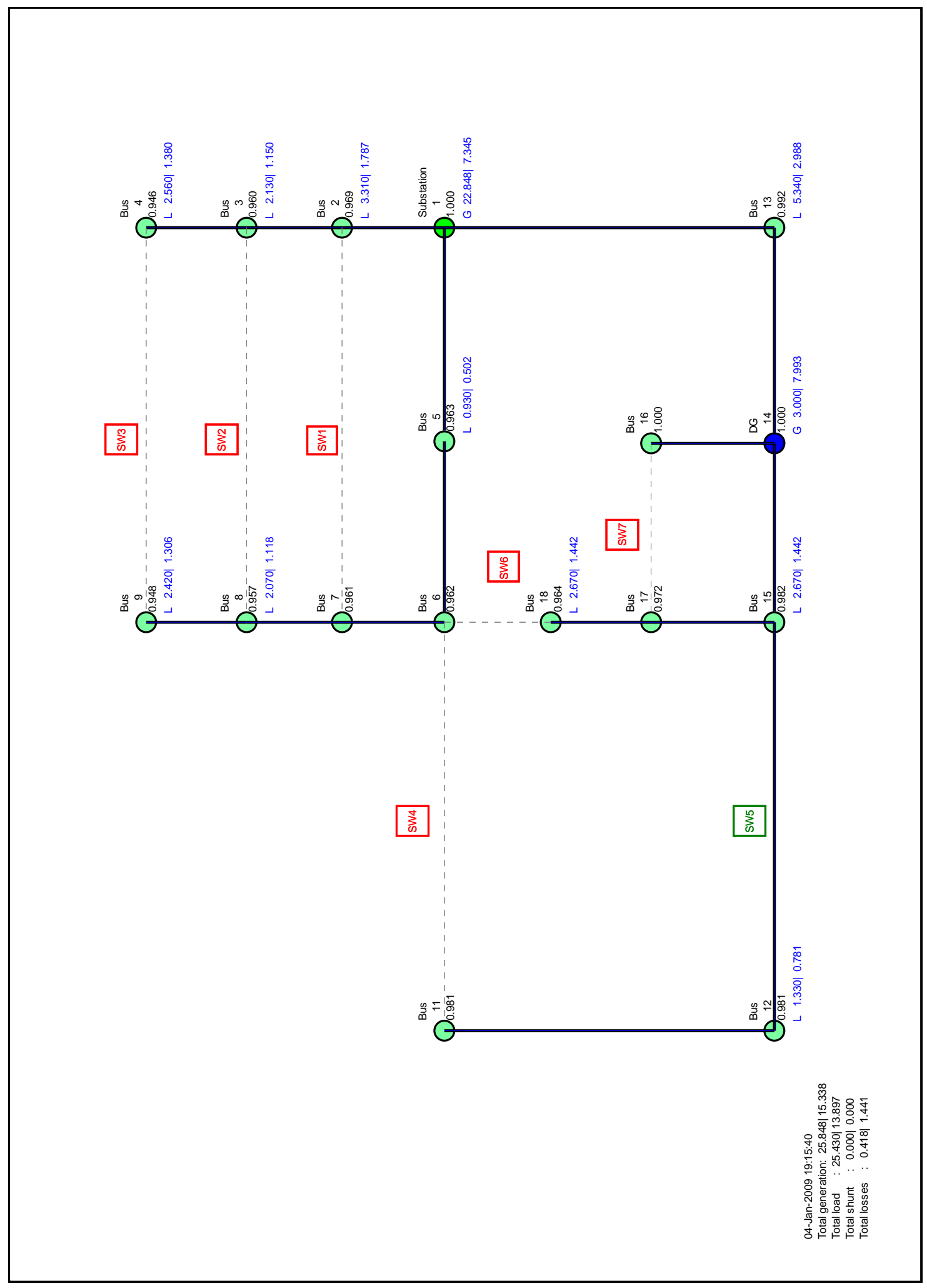

Figure 5-28: MATLAB plot depicting reconfiguration network for fault at lines 9-10 and 10-11. 


\subsubsection{Test Case 7: Special Scenario}

In this section, a special scenario related to Test Case \# 3 is discussed. Let us assume there is a fault at distribution line 1-2. In this particular scenario, the way the reconfiguration algorithm reconfigures the network is modified. From the previous chapter, it is clear that the reconfiguration algorithm searches for second switch for getting voltage support after operating the first switch. In this scenario, the reconfiguration algorithm operates the first switch to reconfigure the network, and then uses the voltage control technique for getting the voltage support. Here the reconfiguration algorithm doesn't search for the second switch for the voltage support. This scenario helps in understanding different formulations of reconfiguration algorithm. The final reconfigured network is shown in Figure 5-29.

The final reconfiguration plot clearly shows the difference between execution of Test Case \# 3 and Test Case \# 7. In Test Case \# 3, two switches $S_{1}$ and $S_{5}$ are operated in order to reconfigure the network and to get the good voltage profile. In the present case, only the switch $S_{1}$ is operated and to build the voltage profile voltage control techniques such as shunt compensation and priority based load shedding are used. In this case, total of 4.5 MVAR of shunt compensation and loads $L_{11}, L_{9}, L_{7}$, and $L_{1}$ are turned off for providing good voltage profile across the network.

If one doesn't want to use the voltage control techniques and want to build the voltage profile using possible switch configurations, then one can adopt the procedure followed in Test Case \# 3. If one is reluctant about switching ON more switchable distribution lines, then one can follow the present case. It's purely one's discretion to choose the way he/she wants to reconfigure the network. Hence, this scenario just visualizes different formulations of fault reconfiguration and their pros and cons. 


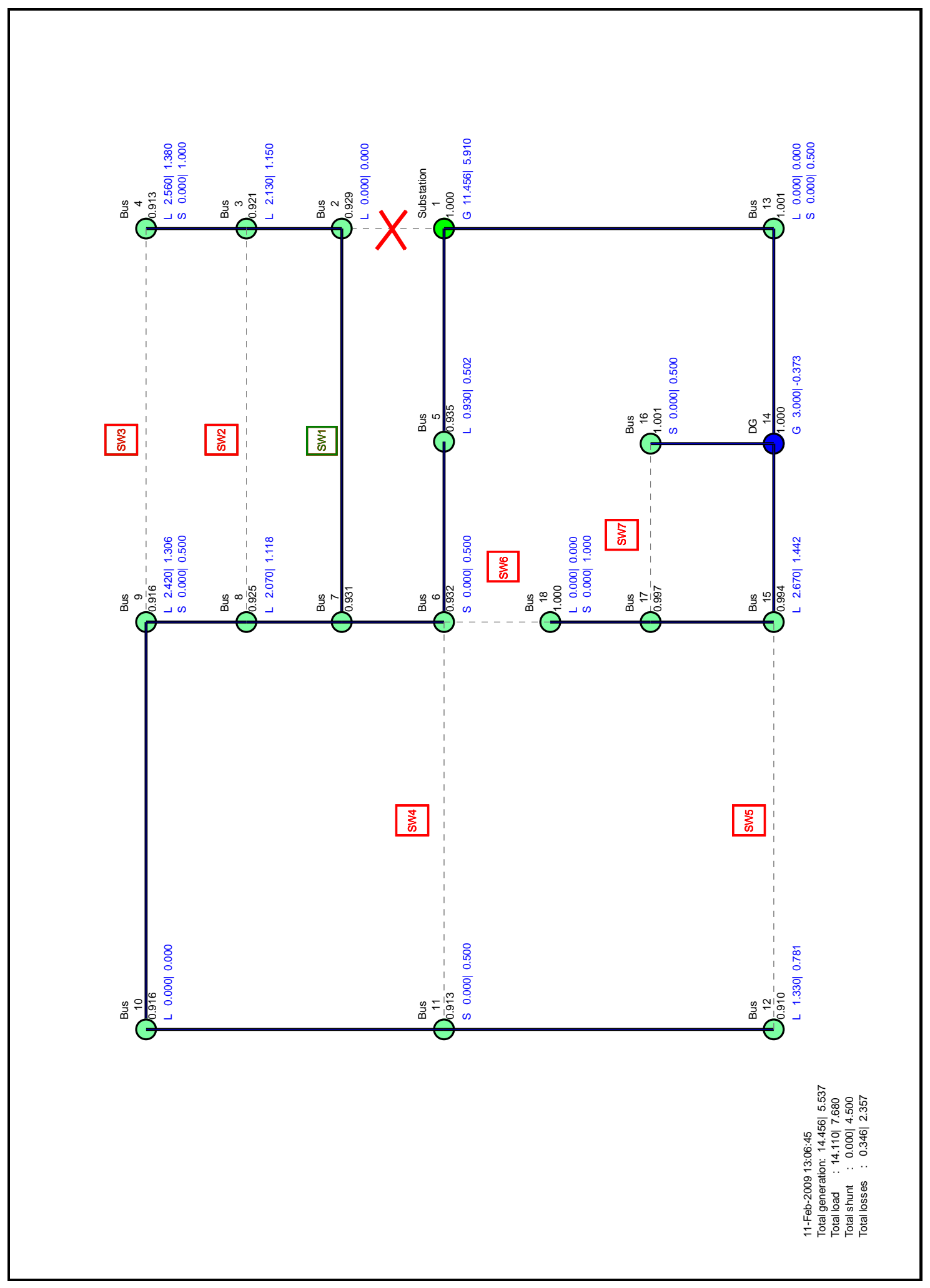

Figure 5-29: MATLAB plot depicting reconfiguration network for fault at line 1-2. 


\subsection{Algorithm Execution Duration}

In this section both the Fault Detection algorithm and Fault Reconfiguration algorithm are tested for their execution durations. Many times the efficiency of algorithm is related to its execution time. The lesser time the algorithm takes to execute certain function, the efficient is the algorithm. The following Figure 5-29 shows the execution time taken for 100 samples of fault detection processes. It is observed that the average time taken for fault detection is $22.4 \mathrm{~ms}$.

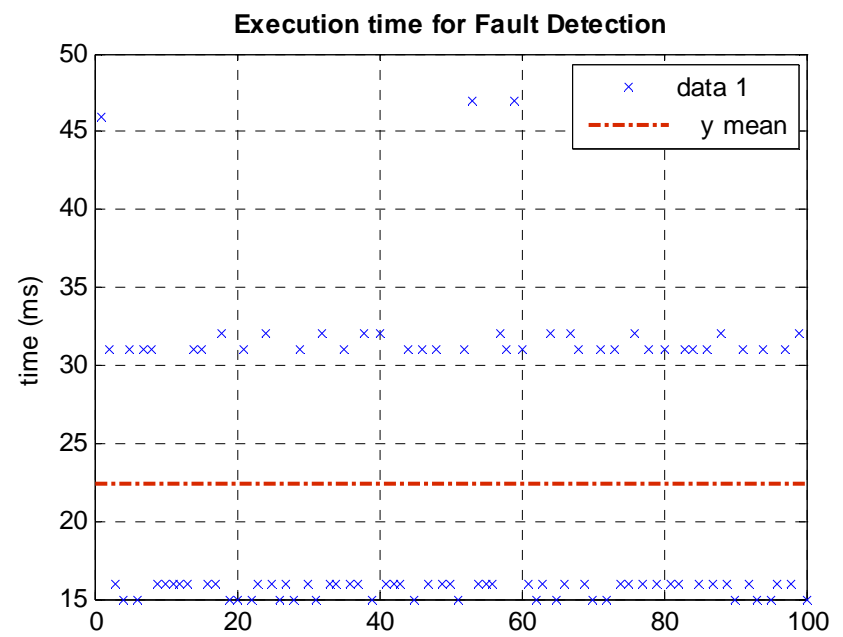

Figure 5-30: Plot of Execution Time for Fault Detection Algorithm [2].

The following Figure 5-30 shows the execution time taken for fault reconfigurations for 50 sample scenarios. The average execution time is $1.8796 \mathrm{sec}$.

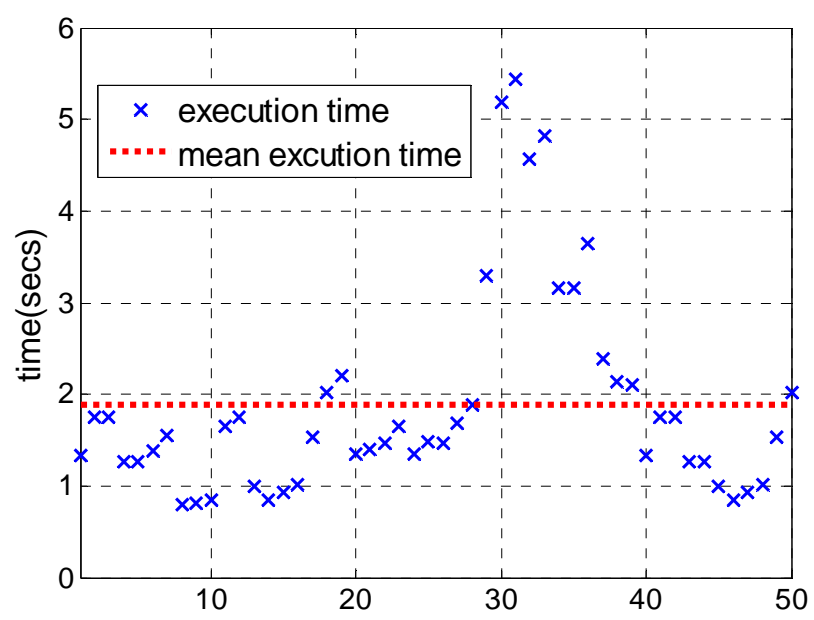

Figure 5-31: Plot of Execution Time for Fault Reconfiguration Algorithm. 
As explained earlier in the chapter, the Global Agent has data base, which keeps record of all the scenarios which happened earlier. When the same scenario happens in the future GAG recalls its experience and reconfigures the circuit based on its experience. Because of this fact the fault reconfiguration execution time is drastically decreased. The same scenarios as of in Figure 5-30, are run again to test the execution times of Fault Reconfiguration algorithm. The following plot shows the execution times for the same scenarios as of in Figure 5-30 with accessing data base.

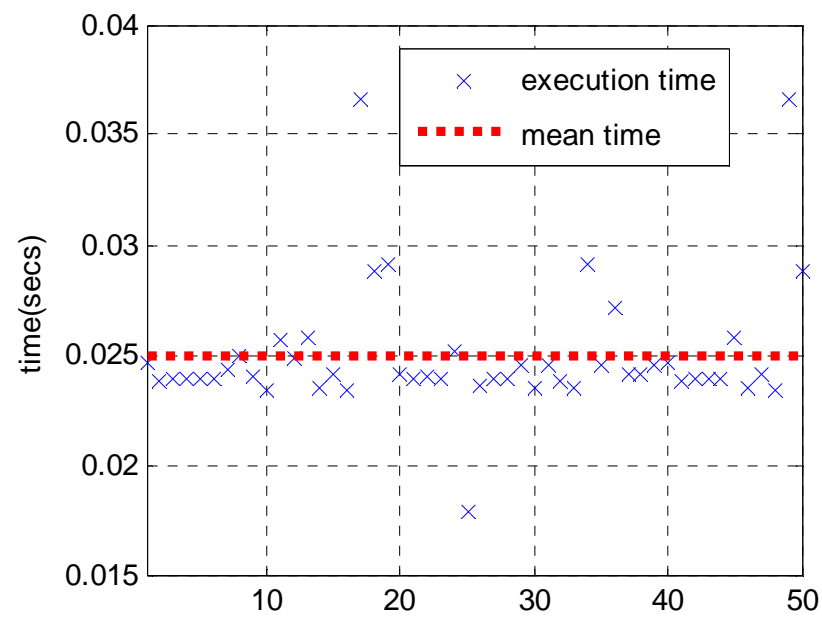

Figure 5-32: Plot of Execution Time for Fault Reconfiguration Algorithm accessing data base.

When GAG accesses its data base to run the reconfiguration, the execution time taken by the algorithm is greatly reduced. It is observed that the average execution time for fault reconfiguration is $25 \mathrm{~ms}$, which sounds a very promising achievement in algorithm development.

These execution times are only concerning to the software implementation. Actual execution times in the real world implementation would be longer than the times specified here. This is due to the software and hardware interface and communication delays. 


\section{Chapter 6}

\section{Conclusion ANd Future Work}

Multi-agent systems are part of Distributed Artificial Intelligence (DAI). For over a decade many researchers have proposed myriad applications of MAS in different engineering fields. Recently MAS are being explored and applied in the area of power engineering. Power systems are becoming more complicated and the present control techniques are insufficient for safer and reliable operation. MAS would be a perfect solution for large and complicated systems like power systems. MAS are autonomous, intelligent, distributed and provide faster solutions for today's power system complex problems.

\subsection{Conclusion}

In this thesis a MAS model is proposed for Fault detection and Fault reconfiguration. The proposed Multi-agent system makes use of both centralized and decentralized methods to make up for the disadvantages of each method. The proposed model of MAS is tested on proto-type distribution network named Circuit of the Future (CoF). The network has three outgoing distribution feeders which supplies number of loads in the distribution network. CoF has number of switchable transmission lines (switches) for the purpose of re-routing the power during fault reconfiguration and restoration of the network.

In the proposed MAS model, there are two hierarchical agents named Local Agents and Global agent. The operation of Local Agents is in decentralized manner where as the 
operation of Global Agent is in centralized manner. Local Agents comprise of Load Agents (LAGs) and Switch Agents (SAGs). A mathematical model based on graph theory is developed for Fault detection in distribution network. Fault detection process is carried out by the communication and coordination of LAGs. Each LAG communicates to its neighboring agent and based on the local information of each LAG the fault location is detected precisely. After the fault detection process LAGs report the fault location to GAG in order to reconfigure and restore the network. GAG has its own database, where it stores all scenarios and will recall these in future in order to speed up the reconfiguration process.

The main objectives of the fault reconfiguration are, to supply the critical loads and to maintain the good voltage profile across the network even in the event of faults. The fault reconfiguration algorithm makes use of shunt compensation and priority based load shedding in order to control the voltage. The fault reconfiguration algorithm proposed in this thesis will choose the best possible switching configuration in regards to voltage profile and real power loss in the system.

The MAS agent architecture is developed using JADE platform for fault detection and reconfiguration. Global Agent will make use of MATLAB reconfiguration algorithm for reconfiguration and restoration of power distribution system. All possible scenarios are tested on the proto-type test system, Simplified CoF. The results obtained are very promising and show superior ability of Multi-agent systems in the field of fault detection and reconfiguration.

Fault detection and fault reconfiguration algorithms proposed in this thesis are very efficient in terms of algorithm execution times. Execution times for both algorithms are tested. It is observed that the average execution time taken by fault detection algorithm is $22.4 \mathrm{~ms}$ and fault reconfiguration algorithm is $1.8796 \mathrm{sec}$. When the same fault scenarios ran from GAG's database, a significant reduction in execution time is noted and the average execution time recorded is $25 \mathrm{~ms}$. 


\subsection{Future Work}

MAS technology is a new area in the field of power engineering and has lot of potential benefits when compared to traditional schemes. The technology is still in research arena, and has greater number of possible extensions. Some of the future extensions to the work cited in this thesis are listed below,

- The key feature of the Multi-agent system is its operation in decentralized manner. In the proposed work, a centralized reconfiguration scheme is used by global agent for circuit reconfiguration and restoration. Hence, a new decentralized agent model for reconfiguration has to be developed.

- Interfacing agent platforms with the already existing power system software is a main technical challenge, which exists even today. There should be a nice interface, in order to apply the Multi-agent systems to the power system and to harness its key benefits. In the proposed work, there is a need of interface between JADE and Power World Simulator. This can be done by developing an interface using $\mathrm{C}++$ language. There is also a need of efficient interface between JADE and MATLAB.

- The fault detection and fault reconfiguration algorithms are developed based on an assumption of radial configuration of the system. In practical, most of the distribution networks have mesh or loop configuration. Hence, a new set of algorithms should be developed to accommodate all types of circuit topologies.

- The fault detection algorithm used in the proposed work makes use of Load agents for fault detection. This algorithm has a disadvantage of not locating faulty links instead it locates faulty sections. Hence, a fault detection algorithm based on node agents should be developed. 
- Finally, a complete MAS architecture should be developed which can cater all the needs of power systems such as, fault detection, fault reconfiguration, voltage control, protection co-ordination, incorporation of distributed generators, learning and reactive power management. 


\section{REFERENCES}

[1] http://www.eei.org/industry issues/industry overview and statistics/history

[2] Koushaly Nareshkumar, "Application of Multi-agents to Power Distribution Systems" M. S. Thesis, West Virginia University, 2008

[3] P. Silpa, "Power Market Analysis Tool for Congestion Management", M.S.Thesis, West Virginia University, 2007

[4] http://en.wikipedia.org/wiki/2003 North America blackout

[5] Fabio Luigi Bellifemine, Giovanni Caire, Dominic Greenwood, "Developing MultiAgent Systems with JADE", Wiley publisher, 2007

[6] G. Weiss, "Multi-agent Systems: A Modern Approach to Distributed Artificial Intelligence," The MIT Press, 2000

[7] M. Wooldridge, N. R. Jennings, "Intelligent agents: Theory and Practice," The Knowledge Engineering Review, Vol. 10, Issue 2, pp 115 - 152, 1995

[8] P. Tulpule, "Multi-agent Approach for Power System Reconfiguration," M. S. Thesis, West Virginia University, 2007

[9] S. Russell and P.Norvig. Artificial Intelligence: A modern approach. Printice-Hall, 1995

[10] F.Hayes-Roth, D.A. Waterman and D.B.Leat, editors. Building Expert Systems, Addison-Wesley: Reading, M.A, 1983 
[11] P.Jackson, Introduction to Expert Systems Addison-Wesley: Reading, M.A, 1986

[12] John R Searle. Speech Acts: An essay in the philosophy of Language. Cambridge U. Press, 1970

[13] The Foundation for Physical Intelligent Agents, http://www.fipa.org

[14] FIPA 97 specifications, version 2.0, ACL, http://www.fipa.org

[15] D. A. Cartes and S. K. Srivastava, "Agent Applications and their future in Power Industry," IEEE Power Engineering Society General Meeting, pp 1 - 6, June 2007

[16] Z. Zhang, J. D. McCalley, V. Vishwanathan and V. Honavar, "Multi-agent System Solutions for Distributed Computing, Communications and Data Integration Needs in Power Industry," IEEE Power Engineering Society General Meeting, Vol. 1, pp $45-49$, June 2004

[17] L. Liu, K. P. Logan, D. A. Cartes and S. K. Srivastava, "Fault Detection, Diagnostics and Prognostics: Software Agent Solutions," IEEE Transactions on Vehicular Technology, Vol. 56, No 4, pp 1613 - 1622, July 2007

[18] R. Isermann, "Process fault detection based on modeling and estimation methods - A survey," Automatica, Vol. 20, No. 4, pp 387 - 404, July 1984

[19] R. Isermann, "Model based fault detection and diagnosis - Status and Applications," Annual Rev. Control, Vol. 29, pp 71-85, 2005

[20] E. Chow and A. Willsky, "Analytical Redundancy and the design of robust failure detection systems," IEEE Transactions on Automatic Control, Vol. 29, No. 7, pp 603 - 614, July 1984 
[21] R. J. Patton and J. Chen, "Advances in Fault Diagnosis using Analytical Redundancy," IEE Proceedings of Integrated Operations Management Control, pp $6 / 1-6 / 12,1993$

[22] K. Huang, S. K. Srivastava, D. A. Cartes and L. Liu, "Agent Solutions for Navy Shipboard Power Systems," IEEE International Conference on Systems Engineering, pp $1-6$, April 2007

[23] C. Angeli, "Online fault Detection Techniques for Technical Systems: A Survey," International Journal in Computer Science Applications, Vol. 1, No. 1, pp 12 - 30, 2004

[24] K. Huang, S. Srivastava and D. Cartes, "Decentralized Reconfiguration for Power Systems using Multi Agent System," 1st Annual IEEE Systems Conference, pp 1 - 6, April 2007

[25] J. M. Solanki, N. N. Schulz and W. Gao, "Reconfiguration for Restoration of Power Systems using Multi-Agent System," Proceedings of the 37th Annual North American Power Symposium, pp 390 - 395, October 2005.

[26] J. M. Solanki, S. Khushalani and N. N. Schulz, "A Multi-Agent Solution to Distribution Systems Restoration," IEEE Transactions on Power Systems, Vol. 22, No. 3, pp 1026 - 1034, August 2007

[27] T. Nagata, H. Sasaki and R. Yokoyama, "Power System Restoration by Joint Usage of Expert System and Mathematical Programming Approach," IEEE Transactions on Power Systems, Vol. 10, No. 3, pp 1473 - 1479, August 1995

[28] T. Nagata and H. Sasaki, "A Multi-agent Approach to Power System Restoration," IEEE Transactions on Power System, Vol. 17, No. 2, pp 457 - 462, May 2002

[29] T. Nagata, N. Iba, Y. Tao and H. Sasaki, "A New Power System Restoration Framework by means of Multi-agent Approach," IEEE/PES Transmission and Distribution Conference and Exhibition, Vol. 3, pp. 1695 - 1700, October 2002 
[30] T. Nagata, Y. Tao and H. Fujita, "An Autonomous Agent for Power System Restoration," IEEE Power Engineering Society General Meeting, Vol. 1, pp 1069 1074, June 2004

[31] T. Nagata, Y. Tahara and H. Fujita, "An Agent Approach to Bulk Power System Restoration," IEEE Power Engineering Society General Meeting, Vol. 1, pp 599 604 , June 2005

[32] T. Nagata, Y. Tao, Y. Tahara and T. Aoyama, "Development of Bulk Power System Restoration Simulator by means of Multi-agent Approach," The $47^{\text {th }}$ IEEE International Midwest Symposium on Circuits and Systems, Vol. 2, pp 337 - 340, July 2004

[33] D. Liu, Y. Chen, G. Shen and Y. Fan, "Multi-agent based Approach for Modeling and Simulation of Bulk Power System Restoration," IEEE/PES Transmission and Distribution Conference and Exhibition, pp 1- 6, 2005

[34] L. M. Tolbert, H. Qi and F. Z. Peng, "Scalable Multi-Agent System for Real-Time Electric Power Management," IEEE Power Engineering Society Summer Meeting, Vol. 3, pp $1676-1679$, July 2001

[35] T. Hiyama, D. Zuo and T. Funabashi, "Multi-agent based Control and Operation of Distribution System with Dispersed Power Sources," IEEE/PES Transmission and Distribution Conference and Exhibition, Vol. 3, pp 2129 - 2133, 2002

[36] Y. Qudaih and T. Hiyama, "Reconfiguration of Power Distribution System using Multi Agent and Hierarchical based Load following Operation with Energy Capacitor System," International Power Engineering Conference, pp. 223 - 227, December 2007

[37] J. A. Hossack, J. Menal, S. D. J. McArthur and J. R. McDonald, "A Multi Agent Architecture for Protection Engineering Diagnostic Assistance," IEEE Transactions on Power Systems, Vol. 18, No. 2, pp 639 - 647, May 2003 
[38] J. Zhang and Q. Liu, "Application of a Multi-Agent Technology in Relaying Protection," International Conference on Power System Technology, pp 1 - 6, October 2006

[39] D. V. Coury, J. S. Thorp, K. M. Hopkinson and K. P. Birman, "An Agent based Current Differential Relay for use with a Utility Intranet," IEEE Transactions on Power Delivery, Vol. 17, No. 1, pp 47 - 53, January 2002

[40] S. J. Park and J. T. Lim, "Modeling and Control of Agent-based power protection systems using supervisors," IEE Proceedings on Control Theory and Applications, Vol. 153, Issue 1, pp 92 - 98, January 2006

[41] Z. Xiangjun, K. K. Li, W. L. Chan and S. Sheng, "Multi-Agents based Protection for Distributed Generation Systems," Proceedings of the IEEE International Conference on Electric Utility Deregulation, Restructuring and Power Technologies, Vol. 1, pp 393 - 397, April 2004

[42] H. Wan, K. K. Li and K. P. Wong, "An Multi-Agent Approach to Protection Relay Coordination with Distributed Generators in Industrial Power Distribution System," Conference Record of the Industry Applications $40^{\text {th }}$ IAS Annual Meeting, Vol. 2, pp 830 - 836, October 2005

[43] H. Wan, K. K. Li and K. P. Wong, "Multi Agent Application of Substation Protection Coordination with Distributed Generators," Proceedings of International Conference on Future Power Systems, November 2005

[44] M. W. David and K. Sakurai, "Protecting the Electric Power Infrastructure with a Mobile Agent Monitoring and Analysis System," Proceedings of International Conference on Information and Communication Technologies: From Theory to Application, pp 497 - 498, April 2008

[45] W. Hu, L. Benedicenti and R. Paranjape, "Mobile Agent Network Management System Performance Study in Frame Relay Network," Proceedings of Canadian Conference on Electrical and Computer Engineering, Vol. 3, pp 1499 - 1504, May 2002 
[46] T. Shono, K. Sekiguchi, T. Tanaka and S. Katavarna, "A Remote Supervisory System for a Power System Protection and Control Unit Applying Mobile Agent Technology," Proceedings of IEEE/PES Transmission and Distribution Conference and Exhibition, Vol. 1, pp 148 - 153, October 2002

[47] H. F. Wang, "Multi-agent Co-ordination for the Secondary Voltage Control in Power System Contingencies," IEE Proceedings on Generation, Transmission and Distribution, Vol. 148, Issue 1, pp 61 - 66, January 2001

[48] S. Gehao, J. Xiuceng and Z. Yi, "Optimal Coordination for Multi-agent based Secondary Voltage Control In Power System," IEEE/PES Transmission and Distribution Conference and Exhibition, pp 1 - 6, 2005

[49] V. Krishna and V C Ramesh, "Intelligent Agents for Negotiations in Market Games, Part 1: Model," Proceedings of PICA97.

[50] V. Krishna and V C Ramesh, "Intelligent Agents for Negotiations in Market Games, Part 2: Application," Proceedings of PICA97.

[51] Hyungna Oh, Tim Mount, "On the First Spike in Summer", Proceedings of the 37th Hawaii International Conference on System Sciences - 2004

[52] Hyungna Oh, Tim Mount, "Testing the Effects of Holding Forward Contracts on the Behavior of Suppliers in an Electricity Auction", Proceedings of the 38th Hawaii International Conference on System Sciences - 2005

[53] Junjie Sun, Leigh Testfatsion, "An Agent-Based Computational Laboratory for Wholesale Power Market Design", IEEE, 2007

[54] Yeung C S K, Poon A S Y, Wu F F, Game theoretical multi-agent modeling of coalition formation for multilateral trades [C]. IEEE Transactions on Power Systems, Volume 14, Issue 3, Aug 1999 929-934. 
[55] Ping Wei, Yongle Yan, Yixin Ni, Yen J, Wu F F, Allocation of tieline costs in power exchange scheduling using a multi-agent approach[C]. IEEE Power Engineering Society Winter Meeting 2001.Volume 3, 2001 1220-1225.

[56] Reinprecht N B, Bones D, Data management for the electricity market[C]. IEEE Computer applications in Power, Volume: 14, Issue: 1, Jan, 2001: 35-38.

[57] A. Aitouche and S. Hayat, "Multi-agent Model using Colored Petri Nets for the Regulation Traffic of an Automated Highway," IEEE Proceedings on Intelligent Transportation Systems, Vol. 1, pp $37-42,2003$

[58] V. Gorodetsky, O. Karsaev, V. Kupin and V. Samoilov, "Agent based Air Traffic Control in Airport Airspace," IEEE/WIC/ACM International Conference on Intelligent Agent Technology, pp 81 - 84, 2007

[59] K. Zafar, S. B. Qazi and A. R. Baig, "Mine Detection and Route Planning in Military Warfare using Multi Agent System," IEEE Proceedings of the $30^{\text {th }}$ Annual International Computer Software and Applications Conference, Vol. 2, pp 327 332, September 2006

[60] S. D. J. McArthur, E. M. Davidson, V. M. Catterson, A. L. Dimeas, N. D. Hatziargyriou, F. Ponci and T. Funabashi, "Multi-Agent Systems for Power Engineering Applications - Part I: Concepts, Approaches, and Technical Challenges," IEEE Transactions on Power Systems, Vol. 22, No. 4, pp 1743 1752, November 2007

[61] S. D. J. McArthur, E. M. Davidson, V. M. Catterson, A. L. Dimeas, N. D. Hatziargyriou, F. Ponci and T. Funabashi, "Multi-Agent Systems for Power Engineering Applications - Part II: Technologies, Standards, and Tools for Building Multi-agent Systems," IEEE Transactions on Power Systems, Vol. 22, No. 4, pp 1753 - 1759, November 2007 
[62] G. Morejon, S. K. Srivastava and D. A. Cartes, "Integrating Virtual Test Bed and JADE Agent Platform using CORBA for Reconfiguration of Shipboard Power System," IEEE Power Engineering Society General Meeting, June 2006

[63] The MathWorks ${ }^{\mathrm{TM}}$, http://www.mathworks.com/products/matlab/description1.html

[64] MATPOWER home page, http://www.pserc.cornell.edu/matpower/

[65] PSERC PowerWeb project, http://www.pserc.cornell.edu/powerweb/

[66] Java Agent Development Framework, http://jade.tilab.com/

[67] F. Bellifemine, G. Caire and D. Greenwood, "Developing Multi-Agent Systems with JADE," John Wliey \& Sons, Ltd., 2004

[68] H. Shaalan, J. Thompson, R. Broadwater, M. Ellis and H. Ng, "Distribution Engineering Tool Features a Flexible Framework," IEEE Transactions on Computer Applications in Power, Vol. 8, Issue 3, pp 21 - 24, July 1995

[69] R. Broadwater, J. Thompson, M. Ellis, H. Ng, N. Singh and D. Loyd, "Application Programmer Interface for the EPRI Distributed Engineering Workstation," Proceedings of IEEE Transactions on Power Systems, Vol. 10, No. 1, pp. 499 505, February 1995

[70] Power World Simulator, http://www.powerworld.com/Default.asp

[71] Wikipedia, http://en.wikipedia.org/wiki/Graph theory 